University of Tennessee Health Science Center

UTHSC Digital Commons

\title{
An Examination of Factors that Influence Health Behaviors among Low Income African American Women with Type 2 Diabetes in Memphis, TN: A Qualitative Inquiry Using Mixed Methods
}

Adole Muruako

University of Tennessee Health Science Center

Follow this and additional works at: https://dc.uthsc.edu/dissertations

Part of the Endocrine System Diseases Commons, Health Services Research Commons, and the Women's Health Commons

\section{Recommended Citation}

Muruako, Adole, "An Examination of Factors that Influence Health Behaviors among Low Income African American Women with Type 2 Diabetes in Memphis, TN: A Qualitative Inquiry Using Mixed Methods" (2014). Theses and Dissertations (ETD). Paper 179. http://dx.doi.org/10.21007/etd.cghs.2014.0221. 


\title{
An Examination of Factors that Influence Health Behaviors among Low Income African American Women with Type 2 Diabetes in Memphis, TN: A Qualitative Inquiry Using Mixed Methods
}

\begin{abstract}
Obesity and type 2 diabetes are increasing health concerns in the United States, particularly among women and minorities. Generally, research has highlighted the health disparities that exist among African American women, especially as these disparities relate to obesity and type 2 diabetes. In Memphis, TN, the ZIP codes of 38106 and 38109 have the highest rates of diabetes hospitalizations in the city. Further, African American women in Memphis, TN have been identified as being most vulnerable to experiencing a potentially avoidable hospitalization due to chronic disease. The purpose of study was to identify factors that may impact African American overweight/obese women with type 2 diabetes in their attempts to lose weight and engage in physical activity, and to identify the specific environmental conditions of 38106 and 38109 that may promote or inhibit weight loss and physical activity efforts.

A mixed-methods approach was utilized, using a combination of individual interviews, objective quantification of ZIP code walkability, and field examinations of area resources that could impact health behaviors. Results showed that consistently positive influences found to impact women's ability to effectively manage their diabetes through weight loss and physical activity included the presence of positive social support via neighbors and family, as well as how connected study participants 'were to their community. Factors that contributed to difficulties in losing weight and engaging and regular physical activity were more frequently reported by study participants and included the presence of substantial generational diabetes, area crime, economic factors, nutrition modification difficulties, and various environmental factors including lack of sidewalks and blight. This study found that in the ZIP codes evaluated, the physical environment plays a crucial but secondary role to social capital and the social environment in encouraging weight loss and physical activity. Additionally, each ZIP code has associated unique aspects that may deter physical activity and adequate health maintenance.
\end{abstract}

\section{Document Type}

Dissertation

\section{Degree Name}

Doctor of Philosophy (PhD)

Program

Health Outcomes and Policy Research

Research Advisor

Shelley White-Means, Ph.D.

Keywords

Environment, Geographic Information Systems, Physical activity, Type 2 diabetes, Vulnerable populations

\section{Subject Categories}

Diseases | Endocrine System Diseases | Health Services Research | Medicine and Health Sciences | Public Health | Women's Health 
An Examination of Factors that Influence Health Behaviors among Low Income African American Women with Type 2 Diabetes in Memphis, TN: A Qualitative Inquiry Using Mixed Methods

\author{
A Dissertation \\ Presented for \\ The Graduate Studies Council \\ The University of Tennessee \\ Health Science Center \\ In Partial Fulfillment \\ Of the Requirements for the Degree \\ Doctor of Philosophy \\ From The University of Tennessee
}

By

Adole Muruako

December 2014 
Copyright (C) 2014 by Adole Muruako. All rights reserved. 


\section{DEDICATION}

This dissertation is dedicated to my husband, without whom this would not have been possible to complete. He not only assisted in inspiring this topic; his encouragement, love, and devotion is unsurpassed, and I truly thank him for his unconditional love and support through this process. I love you.

I would also like to dedicate this work to the many civil rights activists who paved the way before me, so that I may have the opportunity to freely illuminate the disparities that continue to exist in the hopes of furthering our progress as a nation, and a world. 


\section{ACKNOWLEDGEMENTS}

I would first like to acknowledge God, and am truly thankful that my faith in You has soared since the beginning of this process. Through this dissertation my spirituality and faith has broadened substantially, and this process has illuminated the fact that everything happens for a reason and that it is all in Your perfect time. Thank You.

I also acknowledge my mother, father, and brothers, and extended family and friends for their love and encouragement through this process and throughout the years. My father, who also possesses a Ph.D., has been a motivating force and a person that I have looked up to for his work ethic, drive, and unapologetic devotion to excellence. My mother, in her unwavering encouragement for me to "stay the course no matter what", has always been an ear when I've been down, a shoulder to cry on, and a hand to hold, and I'll always be grateful to her.

I gratefully acknowledge the women who contributed their experiences to aid in the conduct of this study. The women interviewed opened their doors up to me, offered me sustenance for my comfort, and themselves provided encouragement for me to continue with this work. They understood its value and importance and were willing to open their hearts and share their experiences, with many of the experiences being private and some painful. I will forever be in their debt for their trust in me, and support and belief in this project. I also thank the community members of 38106 and 38109 for kindly and patiently answering my many questions with interest and concern.

I also acknowledge Dr. Phyllis Betts at the University of Memphis, who has since retired, for giving me permission to use CBANA data in the conduct of this study, as well as TK Buchanan at the University of Memphis for providing invaluable data and GIS guidance pertaining to CBANA Infoworks data. Similarly, I'd particularly like to acknowledge Arjun Rattan and colleagues at the Regional Municipality of Halton in Halton, Canada for granting permission to replicate their study methods to quantify walkability within this study's methods.

I would finally like to acknowledge my committee members. Their diligence, patience, and support through writing this dissertation has been appreciated beyond words. Dr. White-Means, thank you for taking on the role as my advisor and mentor; for being patient with me; and for always being kind and understanding. Your beautiful heart always has shone through, with every conversation we've had. Dr. Russell, thank you for your keen intelligence and attention to detail; for helping to sharpen me and pushing me to be better than I thought I could be especially with regard to Chapter 4; and for allowing me to express my concerns with kindness. Dr. Nouer, thank you for always having a kind word, encouragement, and an open door, and for your input regarding GIS. Dr. Dagogo-Jack, you are an inspiration to me and it was an honor to have you on my committee. Thank you for also always having an open door, for sharing your contacts with me for recruitment, your willingness to listen to my thoughts, and 
lending an invaluable health provider perspective. Dr. Brown, thank you for helping me formulate the beginnings of this dissertation and for pushing me to strive for excellence. 


\begin{abstract}
Obesity and type 2 diabetes are increasing health concerns in the United States, particularly among women and minorities. Generally, research has highlighted the health disparities that exist among African American women, especially as these disparities relate to obesity and type 2 diabetes. In Memphis, TN, the ZIP codes of 38106 and 38109 have the highest rates of diabetes hospitalizations in the city. Further, African American women in Memphis, TN have been identified as being most vulnerable to experiencing a potentially avoidable hospitalization due to chronic disease. The purpose of study was to identify factors that may impact African American overweight/obese women with type 2 diabetes in their attempts to lose weight and engage in physical activity, and to identify the specific environmental conditions of 38106 and 38109 that may promote or inhibit weight loss and physical activity efforts.

A mixed-methods approach was utilized, using a combination of individual interviews, objective quantification of ZIP code walkability, and field examinations of area resources that could impact health behaviors. Results showed that consistently positive influences found to impact women's ability to effectively manage their diabetes through weight loss and physical activity included the presence of positive social support via neighbors and family, as well as how connected study participants' were to their community. Factors that contributed to difficulties in losing weight and engaging and regular physical activity were more frequently reported by study participants and included the presence of substantial generational diabetes, area crime, economic factors, nutrition modification difficulties, and various environmental factors including lack of sidewalks and blight. This study found that in the ZIP codes evaluated, the physical environment plays a crucial but secondary role to social capital and the social environment in encouraging weight loss and physical activity. Additionally, each ZIP code has associated unique aspects that may deter physical activity and adequate health maintenance.
\end{abstract}




\section{PREFACE}

The following quote seems pertinent to this dissertation and should be stated:

"The philosophy of one century is the common sense of the next." - Henry Ward Beecher

It is the author's sincere hope that this dissertation is read with an open mind, and an open heart. Ultimately, what the author found was, in her humble opinion, simply common sense - we cannot solve social problems unless we adopt a unified perspective, a sincere desire to understand the inner mechanistic workings of others, and a comprehensive, solid, and consistent approach. Although the problems of discrimination encompass a long and arduous history in the United States, the tendency for integration and dissolution of social isolation to, in the long run, produce economic prosperity, psychological well-being, increased opportunity for change and growth, personal empowerment, and overall good will simply cannot continue to be ignored. While individual positive intention and balanced perspective unfortunately cannot be enforced through public policy, as neither can fear of the unknown be completely banished, perhaps the reiteration of love and unity principles - and the realization of the inevitability of these on better overall outcomes - will eventually lead to substantive and long-lasting change. 


\section{TABLE OF CONTENTS}

CHAPTER 1. INTRODUCTION AND OVERVIEW

The Historical Significance of Poverty, Racial Discrimination, Neighborhood

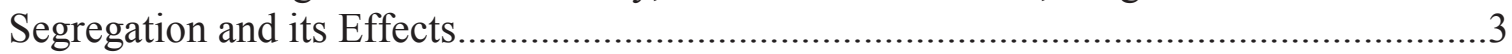

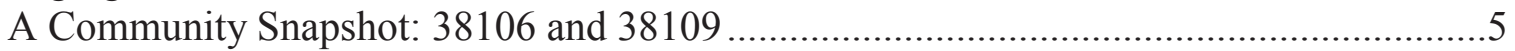

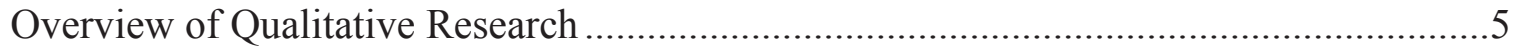

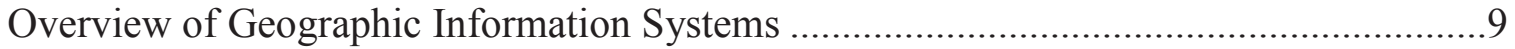

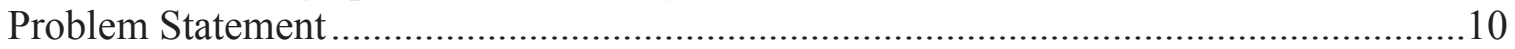

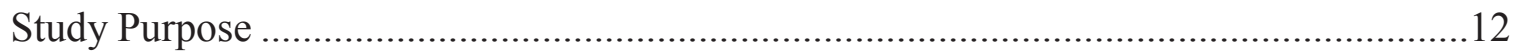

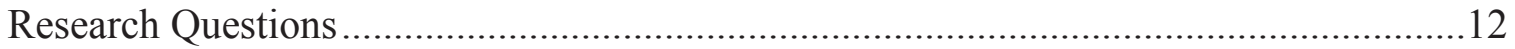

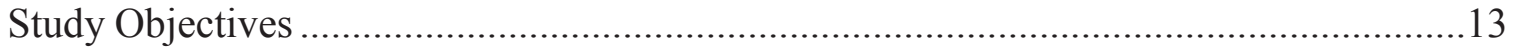

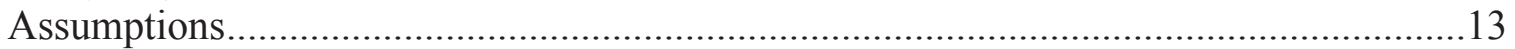

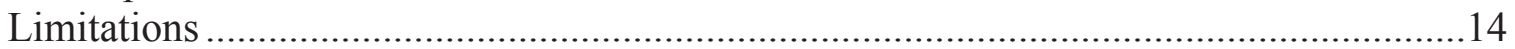

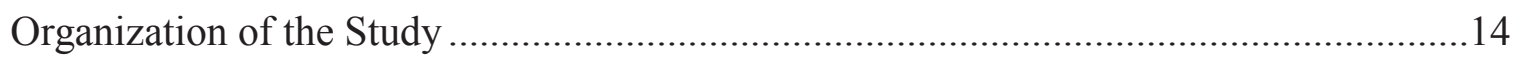

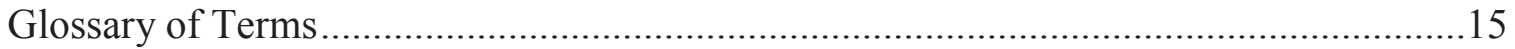

CHAPTER 2. LITERATURE REVIEW .................................................................17

Part 1: Obesity Considerations.......................................................................................17

Obesity and type 2 diabetes demographic information and economic

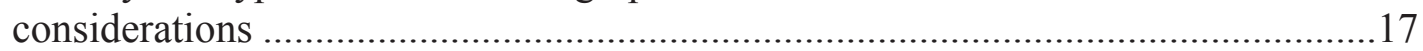

Obesity, type 2 diabetes, and hypertension..............................................................19

Obesity, type 2 diabetes, African American women in the context of poverty,

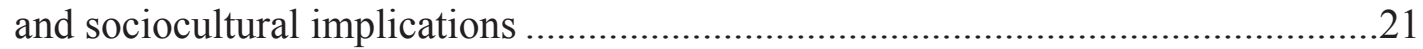

Weight loss outcomes in type 2 diabetes ...........................................................26

Part 2: Physical Activity, Obesity and Considerations of Environmental Circumstance ..28

Physical activity and obesity ...............................................................................28

The connection between physical activity and environment ....................................29

Neighborhoods and their effects on health in the literature .......................................32

Part 3: The Socio-Ecological Model, a Review of the Literature, Conceptual

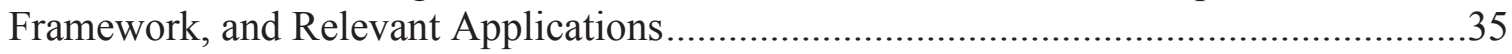

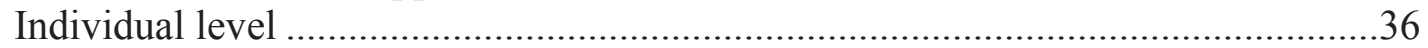

Organizations and public policy level................................................................36

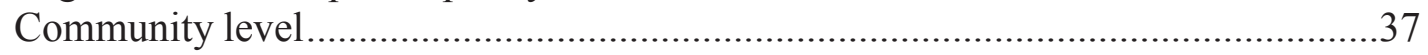

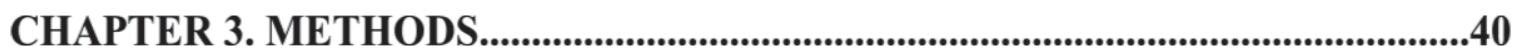

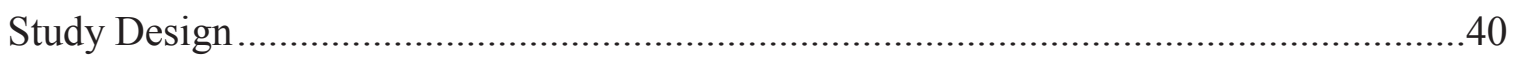

Study Sample Composition....................................................................................40

Data Collection and Instrumentation ......................................................................42

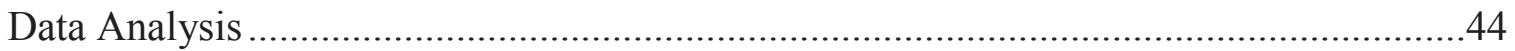

Generating codes and behavior domains from interview responses, phase 1

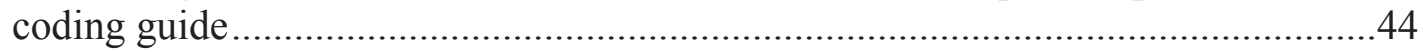

Categorizing codes into themes using behavior domains, phase 2 coding guide .....46

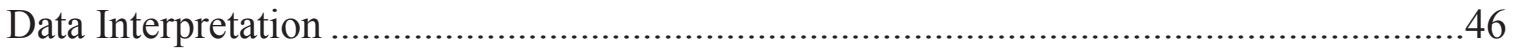

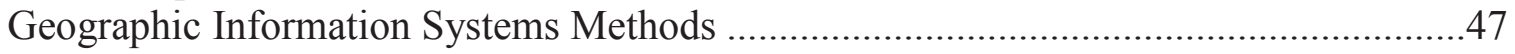


Demographic Information.

Part 1: Identified Major Factors Affecting Weight Loss, Physical Activity, and Health Maintenance

Part 2: An Analysis of Research Questions 1 and 2 in the Context of Identified

Themes

Weight Loss Strategies Employed by the Study Population

Weight loss strategies - Nutrition

Weight loss strategies - Exercise.

Weight loss strategies - Medications and effects on weight loss and health maintenance

Weight loss strategies - Attending diabetes management classes

Sociocultural Factors that Encourage and Discourage Weight Loss and Health

Maintenance . .70

Sociocultural factors - Nutrition.................................................................. 70

Sociocultural factors - Generational diabetes.................................................. 71

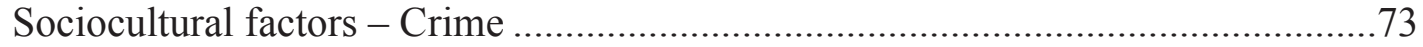

Sociocultural factors - The effects of community and social support on health,

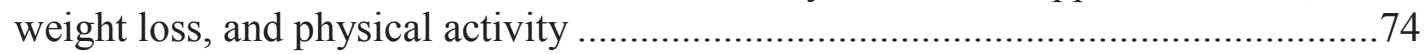

Influences of the Physical Environment that Encourage and Discourage Weight Loss

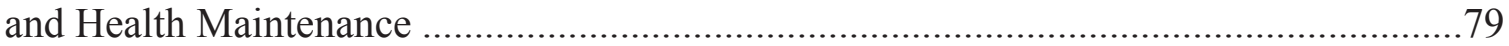

Encouraging environmental influences - Parks.................................................79

Encouraging environmental influences - Mobile markets ......................................80

Discouraging environmental influences - Stray animals......................................8

Discouraging environmental influences - Lack of diabetes resources ....................81

Discouraging environmental influences - Lack of sidewalks in 38109 ...................81

Economic Factors that Affect Health Maintenance .......................................................82

Part 3: An Analysis of Environmental and Structural Barriers in Achieving Weight

Loss Using Geographic Information Systems - ZIP codes 38106 and 38109 .................84

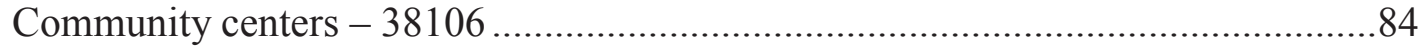

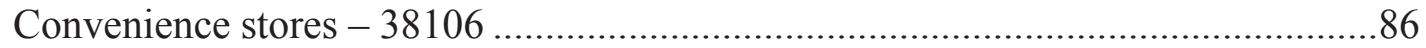

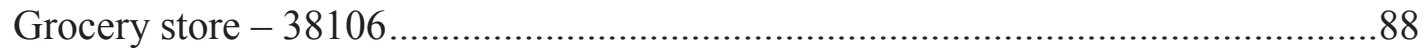

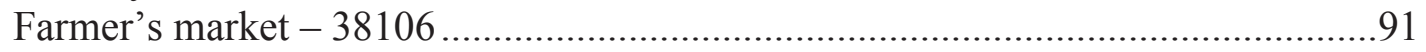

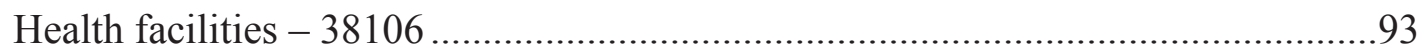

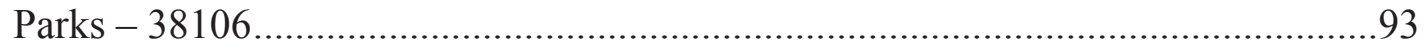

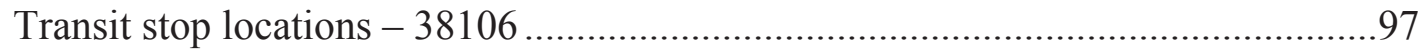

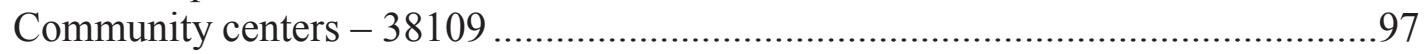

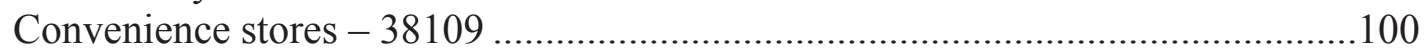

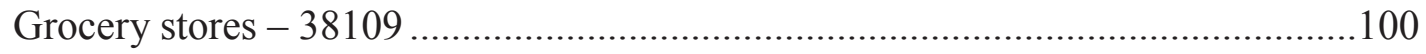

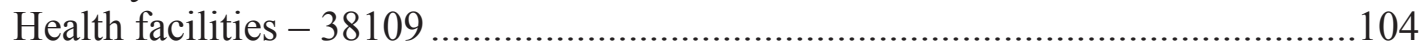

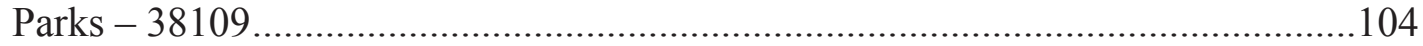

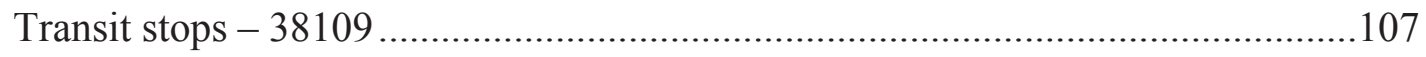

Part 4. An Analysis of 38106 and 38109: A Comparison of Participant Reports and Field Observations of Major Environmental Characteristics that Affect Physical Activity 


\section{CHAPTER 5. DISCUSSION OF FINDINGS, POLICY IMPLICATIONS,}

STUDY LIMITATIONS, FUTURE DIRECTIONS, AND CONCLUSIONS...........112

Introduction.

A Discussion of Sociocultural and Economic Factors that Affect Health Maintenance, Weight Loss, and Physical Activity among African American Women in the 38106 and 38109 ZIP codes

A Discussion of Environmental Factors that Affect Health Maintenance, Weight

Loss, and Physical Activity among African American Women in 38106 and 38109 115

Policy Implications

Limitations

Future Research Directions

Conclusion

LIST OF REFERENCES

APPENDIX A. PHOTOGRAPHS OF 38106 AND 38109 EXHIBITING

POTENTIAL BARRIERS TO PHYSICAL ACTIVITY.

APPENDIX B. RECRUITMENT LETTER

APPENDIX C. RECRUITMENT FLYERS

APPENDIX D. POTENTIAL PARTICIPANT SCREENING

QUESTIONNAIRE.

APPENDIX E. SEMI-STRUCTURED INTERVIEW QUESTIONNAIRE 151

APPENDIX F. CONSTANT COMPARISON CHECKLIST. 153

APPENDIX G. INSTITUTIONAL REVIEW BOARD FORMS 154

VITA. 157 


\section{LIST OF TABLES}

Table 1-1. Demographic information of 38106 and 38109 ZIP codes.........................6

Table 1-2. Comparison of crime rate indexes for 38106, 38109, Tennessee, and the United States. ................................................................................ 7

Table 2-1. Table depicting adult obesity rates by ethnicity - NHANES 2011-2012....20

Table 3-1. Resources of interest and associated walking distance criteria.

Table 4-1. Demographic characteristics and related variables of study respondents.....53

Table 4-2. $\quad$ Study population statistics, overall sample ..............................................54

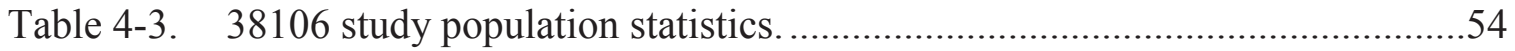

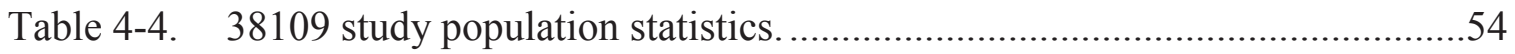

Table 4-5. Factors that positively influence health status and weight loss, and dimensions.

Table 4-6. Factors that negatively influence health status and weight loss, and dimensions identified.

Table 4-7. 38109 convenience store identifiers and labels.

Table 4-8. A comparison of major environmental influences reported that affect physical activity and health maintenance in 38109 and 38106 ZIP codes 


\section{LIST OF FIGURES}

Figure 1-1. Diabetes hospitalization rates in Shelby County, TN................................2

Figure 3-1. Interview and GIS data triangulation.

Figure 3-2. Eligibility determination, informed consent, and interviewing procedures.

Figure 3-3. Model used to generate total ZIP code population.

Figure 3-4. Model used to generate service area polygons.

Figure 4-1. Workflow of recruitment and constant comparison throughout the data collection process.

Figure 4-2. Residents in 38106 within 10 minute walking distance to community centers.

Figure 4-3. Residents within 38106 within 5 minute walking distance to convenience store locations

Figure 4-4. Residents of 38106 within 10 minute walking distance to area grocery store.

Figure 4-5. Residents in 38106 within 10 minute walking distance of the area farmers market.

Figure 4-6. Residents of 38106 within 15 minute walking distance from health facilities.

Figure 4-7. Residents of 38106 within 10 minute walking distance of area parks.

Figure 4-8. Residents of 38106 within a 5 minute walking distance from area transit stops.

Figure 4-9. Residents of 38109 within 10 minute walking distance from area community centers.

Figure 4-10. Residents of 38109 within 5 minute walking distance from area convenience stores.

Figure 4-11. Residents of 38109 within 10 minute walking distance to area grocery stores.

Figure 4-12. Residents of 38109 within 15 minute walking distance from area health facilities. 
Figure 4-13. Residents of 38109 within 10 minute walking distance of area parks........106

Figure 4-14. Residents of 38109 within 5 minute walking distance from transit stops. .109

Figure 5-1. Potential determinants of physical activity and health maintenance within the study population.....................................................................118 


\section{CHAPTER 1. INTRODUCTION AND OVERVIEW}

Obesity and type 2 diabetes are rapidly increasing health concerns in the United States, particularly among women and minorities. A vast quantity of research has illuminated the health disparities that exist among African American women, especially as the disparities relate to obesity and type 2 diabetes. Zhang, Wang, \& Huang (2009) found that racial and ethnic disparities in diabetes prevalence have become more pronounced among normal and overweight groups as well. The literature relating health disparities to social context also posits that individuals (regardless of race) living in dissimilar neighborhoods experience different health risks. Research suggests that race differences in health may arise from race differences in social and environmental exposures driven by residential segregation (Talen \& Shah, 2007).

The Consortium for Health Education, Economic Empowerment, and Research in Memphis, TN (2010) published a map (shown in Figure 1-1) of diabetes hospitalization rates in Shelby County, TN. It was shown that the ZIP codes 38109 and 38106 have among the highest rates of diabetes hospitalizations in Shelby County, suggesting that aggregated factors relating to the proximal environment may have an influence on the health disparities that exist in these areas. Additionally, as seen within these ZIP codes, it has been readily demonstrated that areas with high crime rates, high rates of poverty, and predominantly African American communities generally exhibit poorer health outcomes.

Poor perception of and the presence of neighborhood issues potentially poses severe restrictions on individuals with diabetes, among whom clinical management is required to maintain adequate glycemic control, blood pressure, and lipid control. These management activities require substantial self-regulation including a healthy diet, regular physical activity, self-monitoring of blood glucose, and adherence to medications (Renders et al, 2001). Neighborhoods with environmental issues and barriers including crime and lack of access to resources can obstruct an individual from actively engaging in healthy behaviors. Additionally, lack of access to public transportation could potentially affect participation in appropriate diabetes care by making it difficult to reach appropriate healthcare resources. In several studies, perceived neighborhood problems were associated with more physical inactivity and symptoms and risk of functional loss among older adults (Balfour \& Kaplan, 2002). Even with these known factors, the question remains - despite environmental barriers to adequate health maintenance that may be present in high poverty areas, are there factors that nevertheless can encourage physical activity and weight loss?

Quantitative studies have been conducted that have measured the strength of association between subjective neighborhood measures and behavior. One crosssectional analysis of diabetic adults measured perception of neighborhood problems and associations with health behaviors and outcomes using a summary score of participants' ratings of crime, trash, litter, lighting at night, and access to exercise facilities, transportation, and supermarkets; this study found that neighborhood problems were strongly associated with more smoking and higher blood pressure (Gary et al, 2008). 


\section{Legend}

Sheby Courty Inters tale Health Care Facilities Map Diabetes Hosp. Rate/10k $3.55 \cdot 8.04$ 8.05: 11.12

$11.13 \cdot 26.20$

$26.21 \cdot 38.83$ 389.5386

\section{0k}
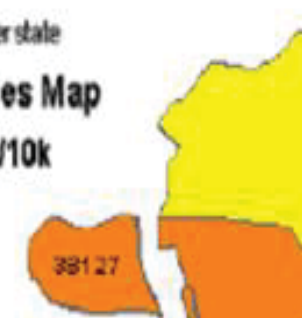

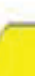

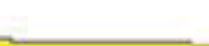

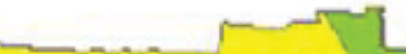

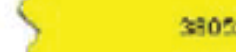

003 8

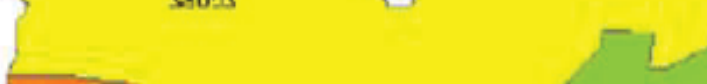

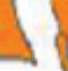

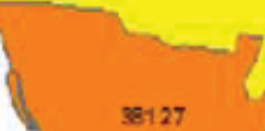

38127
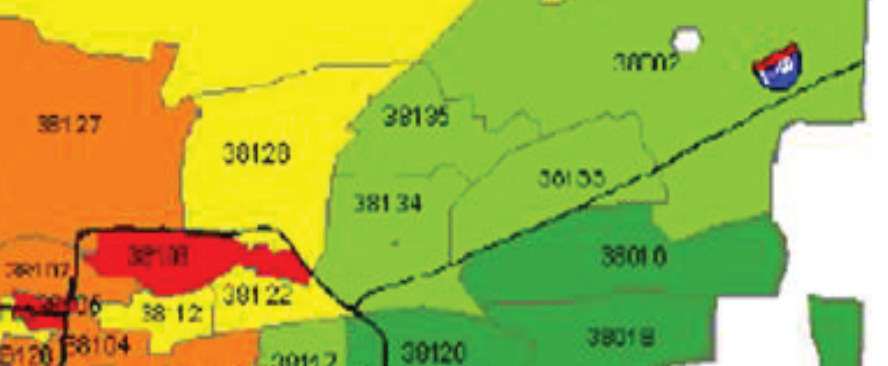

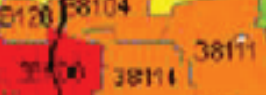

2011?

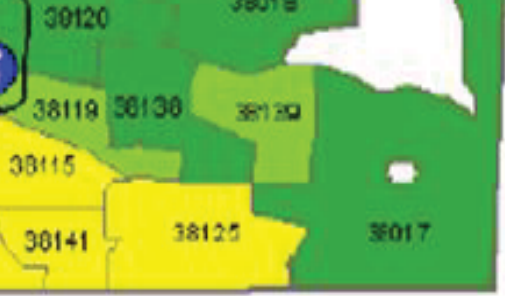

Figure 1-1. Diabetes hospitalization rates in Shelby County, TN.

Reprinted with permission. Consortium for Health Education, Economic Empowerment, and Research. (2010). Diabetes hospitalization rates in Shelby County, TN. Cheerleader, February 2010. 
In the aforementioned study however, neighborhood problems within this study were reported and analyzed as an aggregated measure, prohibiting specificity regarding which neighborhood problems were associated with each outcome. Even with quantitative efforts to measure perceptions of neighborhood and health behaviors, how culture affects individual health behavior may be best answered qualitatively. To more deeply investigate these issues, particularly as they relate to weight loss, physical activity, and health maintenance among impoverished groups, individual considerations of these areas' physical and geographical characteristics, the culture, and resident assumptions that exist are pertinent to creating long-term and sustainable solutions relevant to these specific communities.

\section{The Historical Significance of Poverty, Racial Discrimination, Neighborhood Segregation and its Effects}

According to Sampson and Morenoff (2002), neighborhoods have been defined as a subsection of a larger community - a collection of both people and institutions occupying a spatially defined area influenced by ecological, cultural, and sometimes political forces. They go on to postulate that the local community is best thought of not as single entity, but rather as a grading of progressively more inclusive residential groups (Sampson \& Morenoff, 2002). Most social scientists and virtually all studies of neighborhoods assessed rely on geographic boundaries defined by the Census Bureau or other administrative agencies, but also offer imperfect operational definitions of neighborhoods for research and policy, especially when failing to incorporate the "unspoken" customs of populations (Sampson \& Morenoff, 2002).

There is a substantial body of work regarding the causes of urban poverty from multiple perspectives, including sociology, economic theory, historical discrimination, and even public policy causes. A paper written in 1998 by Michael Teitz and Karren Chapple eloquently outlines eight potential causes of inner-city poverty. They point out that residents living in areas that are isolated, segregated, and crime-ridden are characterized by low labor-market attachment, low educational achievement, and a wide range of socially reproved behaviors, including welfare dependency, drug use, singleparent households, and participation in the underground economy (Teitz \& Chapple, 1998), each of which can ultimately contribute to poor health status. Additionally, sociologists and anthropologists have been effective in capturing the study of life situations of people in poverty, where living entails functional adaption to difficult environmental circumstances (Teitz and Chapple, 1998).

The historical significance of racial segregation within the context of poverty and health outcomes holds noteworthy implications - particularly from the perspective of highlighting that although the circumstances differ, persons in poverty are ultimately no different than the rest of the population. There are psychological implications of discrimination and segregation that far outreach generalized policy interventions and public policy initiatives. In order to not only define the circumstances of the population, but create long-lasting, sustainable solutions, the requirement for a vast reevaluation of 
how persons in poverty are framed is an inevitable course. This alludes to an "insideout" perspective, where connecting to the populations of interest to gain a deeper understanding may generate the most substantive changes. Understanding the community within the context of health outcomes involves its consideration on multiple levels including the culture, concerns, and relationships, which is crucial to developing a comprehensive understanding of the implications of the health outcomes of its residents. Further, according to both American Anthropological Association and the American Association of Physical Anthropologists, race is socially constructed and has little to do with the biological/genetic groupings of classifying human beings (Darity $\mathrm{Jr}$, et al, 2010). This means genetics plays an almost inconsequential role in race health disparity in comparison to how the social and physical environment affects race and health outcomes.

In aiming for a general contextual understanding of how the community may affect the health of women with type 2 diabetes, a picture of the community at a particular point in time can be obtained to provide a framework for individual decisions made, as well as interventions that can be implemented to bring about change (Community Tool Box, 2014). Having the proper background information on community and environmental concerns, especially when evaluating patterns of poor outcomes from an aggregated perspective, can assist in gaining a general idea of the community's strengths and the challenges encountered. This enables the capture of unspoken influential rules and norms, and offers assistance in understanding the character of various areas and the invisible borders that exist among various groups and neighborhoods, which can be extremely important for the physical safety of those working and living in the community (Community Tool Box, 2014). Familiarity with the community can allow policy makers and organizations that wish to design interventions to converse intelligently with residents about community issues. This can serve to establish trust with community members, allowing for effective information dissemination with relevant organizations and coalitions that work in the community to create substantive change.

Important considerations in understanding communities and opportunities present are the existence of stores and other businesses within walking distance of residential areas, considerations of public transportation, as well as topography, infrastructure, open space and greenery, air and water considerations (Community Tool Box, 2014). Additionally, the identification of general policy regarding services to rural and/or poor populations, and whether the layout of the community creates barriers to access and social division can be examined as well. The physical layout of the community provides information and a guide for examining community member responses and references to various areas and neighborhoods, as well as obtaining a sense of the living conditions of the population (Community Tool Box, 2014). 


\section{A Community Snapshot: 38106 and 38109}

Table 1-1 and Table 1-2 depicts a comparison of environmental measures and demographic information for the 38106 and 38109 communities. For the purposes of this study, ZIP code and community will be considered synonymous. Both of these ZIP codes are predominantly African American (95.91\% and 96.16\%, respectively), both have reported high crime indexes, lower than average median household incomes, and relative poverty rates at $25.56 \%$ and $22.44 \%$, respectively. Data from the Center from Community Building and Neighborhood Action (2010) was collected at the neighborhood level and depicts neighborhood resources and assets, social issues, demographic information, housing condition and neighborhood amenities. A direct comparison of these two neighborhoods, also identified as being within ZIP codes demonstrated to have high rates of diabetes hospitalizations, shows generally high rates of blight (poor visual, psychological, and physical effects of living among empty lots, buildings and condemned houses) higher poverty rates, lower median household income, and lower median housing prices when compared to Shelby County. Although the areas are similar in some measures, for other measures the areas differ. The rate of blight is lower in the Westwood/38109 community; the population of 38109 is almost double the population of 38106; the poverty rate in 38109 is slightly lower and the median household income is higher in the 38109 ZIP code.

\section{Overview of Qualitative Research}

Qualitative research is a term with varying meanings in health services research. Green and Thorogood (2004) suggest that the most basic way to characterize qualitative research is that it aims generally to seek answers to questions about the 'what', 'how', or 'why', of a phenomenon, rather than questions about 'how many', or 'how much'. For this proposal's purposes, qualitative research is defined as research devoted to developing an understanding of human systems and perception related to the obesity and type 2 diabetes health states among low-income women and weight loss, expressed by their own experiences and in their own words. Qualitative research typically uses interviews and observations to elucidate such mechanisms, but can also utilize case studies, surveys, and historical and document analysis (Green \& Thorogood, 2004; Rabjee, 2004; Ritchie \& Spencer, 1994). Qualitative methods probe underlying realities of phenomena.

Quantitative methods generalize, categorize, and can, though helpful, eliminate the complexity of the human experience. Qualitative methods allow the identification of promising directions and can lead to the discovery of important information. While the information obtained through qualitative methods is often subjective, it is also often identified as such, and can be examined accordingly (Green \& Thorogood, 2004).

Qualitative research attempts to be open to the subjects' perceptions of "what is"; it should generally be bound by the values and world views of the subjects (Denzin \& Lincoln, 2008). Specifically, use of naturalistic inquiry as a qualitative tool focuses on how people behave when absorbed in genuine life experiences in natural settings (Frey, Boton, \& Kreps, 1999). 
Table 1-1. Demographic information of 38106 and 38109 ZIP codes.

\begin{tabular}{|c|c|c|c|}
\hline Environmental Measures $^{+}$ & 38106 & 38109 & Shelby County \\
\hline Blight rate* & $34.56 \%$ & $24.89 \%$ & $22.06 \%$ \\
\hline Parks/trails & 7 & 12 & ------ \\
\hline $\begin{array}{l}\text { Recreational facilities/commur } \\
\text { centers }\end{array}$ & 3 & 2 & ------- \\
\hline Grocery stores** & 1 & 8 & ------ \\
\hline \multicolumn{4}{|c|}{ General Area Demographic Information } \\
\hline Population (2012) & $\begin{array}{c}\mathbf{4 6 , 5 9 4} \\
\text { Black: } 96.15 \% \\
\text { White: } 2.39 \% \\
\text { Other } 1.22 \%\end{array}$ & $\begin{array}{c}\mathbf{2 7 , 2 2 2} \\
\text { Black: } 95.91 \% \\
\text { White: } 2.09 \% \\
\text { Other: } 1.47 \%\end{array}$ & $\begin{array}{c}\text { 940,763 } \\
\text { Black: } 63.3 \% \\
\text { White: } 29.4 \% \\
\text { Other: } 7.3 \%\end{array}$ \\
\hline Poverty rate $* * *$ & $22.44 \%$ & $25.56 \%$ & $15.95 \%$ \\
\hline Median household income ${ }^{* * *}$ & $\$ 35,733$ & $\$ 26,364$ & $\$ 40,081$ \\
\hline Median housing price $* * *$ & $\$ 54,400$ & $\$ 52,700$ & $\$ 125,250$ \\
\hline
\end{tabular}

Notes: + Use and substantiation of environmental measures in the literature: Kirtland, K.A., Porter, D.E., Addy, C.L, Neet, M.J., Williams, J.E., Sharpe, P.A., et al. (2003). Environmental measures of physical activity supports: Perception versus reality. American Journal of Preventive Medicine, 24, (4), 323-331.

*Information obtained from CBANA Infoworks Memphis Neighborhood Profiles.

$<$ http://cbana.memphis.edu/NeighborhoodProfiles.php $>$.

**Only includes those that provide fresh fruits and vegetables for purchase. Data obtained from "YellowPages -"

$<$ www.yellowpages.com/memphis-tn/>.

***Information obtained from Shelby County via US Census data. (2012) http://www.usa.com/shelby-county-tn.htm. 
Table 1-2. Comparison of crime rate indexes for 38106, 38109, Tennessee, and the United States.

\begin{tabular}{lcccc}
\hline 2010 Crime Risk Index & $\mathbf{3 8 1 0 6}$ & $\mathbf{3 8 1 0 9}$ & Tennessee & $\begin{array}{c}\text { United } \\
\text { States }\end{array}$ \\
& & & & 133 \\
Total Crime Risk & 438 & 445 & 111 & 100 \\
Murder Risk & 544 & 486 & 104 & 100 \\
Rape Risk & 379 & 405 & 115 & 100 \\
Robbery Risk & 730 & 655 & 185 & 100 \\
Assault Risk & 246 & 296 & 144 & 100 \\
Burglary Risk & 417 & 426 & 120 & 100 \\
Larceny Risk & 226 & 169 & 97 & 100 \\
Motor Vehicle Theft Risk & 473 & 530 & & 100 \\
\hline
\end{tabular}

Reprinted with permission. Source: The Federal Bureau of Investigation. (2010) Crime Statistics for 38106, 38109, Tennessee and Shelby County. Accessed April 24, 2014. <http://www.fbi.gov/stats-services/crimestats>.

Notes: "Crime Risk Index (100 = National Average): Index score for an area is compared to the national average of 100 . A score of 200 indicates twice the national average total crime risk, while 50 indicates half the national risk.

Crime Indexes are based on numerous current and historical datasets as well as proprietary modeling algorithms which estimate values at more granular geographic levels when specific data is either unavailable or impractical to aggregate" (Federal Bureau of Investigation, 2010). 
Typical assumptions guiding naturalistic inquiry include the development of context-specific statements about the multiple, constructed realities of key participants. Characteristics of the naturalistic research process include the study of phenomena of interest in situ, researcher placement in context as the primary "instrument" to collect data, and use of an array of interpretive techniques which seek to describe, decode, translate, and come to terms with the meaning of a naturally occurring phenomena (Frey, Boton \& Kreps, 1999). Furthermore, naturalistic inquiry is best when multiple methodological procedures are used to produce various and divergent constructions of reality that exist within a context (Frey, Boton, \& Kreps, 1999). Lincoln and Guba (1985) stress dependability and consistency to reflect what was seen in the data. Reliability of data gathered is evaluated in terms of the researcher's position, sampling method, triangulation, and an audit trail describing the research process in detail.

The research questions in qualitative research often evolve as the study does, because the researcher wants to know "What is happening," and should not want to bias the study by focusing the investigation too narrowly (Denzin \& Lincoln, 2008). Additionally, it is not necessarily assumed that the findings of one study may be generalized easily to other settings (Green \& Thorogood, 2004).

Mixed-methods design (studies that incorporate both quantitative and qualitative attributes in the study design) is noted to be directly related to classic quantitative methods (Ritchie \& Spencer, 1994), but also is noted to take pure qualitative methods out of its natural element. The 'exploration' vs. 'confirmation' nature of the mixed-methods approach is a tentative recent development of qualitative inquiry, as suggested by Denzin and Lincoln (2008); however, it does bring forth the opportunity to explore weight loss among low-income women from both the quantitative and qualitative perspectives. Furthermore, triangulation is particularly relevant to naturalistic inquiry design, because it provides various and divergent constructions of reality that exist within a context of study (Frey, Boton, \& Kreps (1999). Triangulation mixed-methods designs are generally used to converge multiple types of data; to transform data; to validate quantitative data; and to explore multilevel topics (Creswell \& Plano-Clark, 2007). Generally, triangulation involves concurrent data collection, where weighting of both qualitative and quantitative components are equal, and data is merged during the interpretation or analysis (Creswell $\&$ Plano-Clark, 2007). Silverman (2008) further suggests that using qualitative research to explore a particular topic in order to set up a quantitative study is one main way to effectively combine quantitative and qualitative research. This study seeks to first explore low-income women's descriptions of weight loss independent of environmental reflections using individual interviewing qualitative techniques, and then to potentially link weight loss and physical activity effort to proximal resources and neighborhood environmental considerations using Geographic Information Systems.

The interview is the most widely utilized methods of producing data in qualitative research (Green \& Thorogood, 2004). In-depth interviewing is a major investigative strategy used in naturalistic inquiry that involves conducting intensive individual interviews with a small number of respondents to explore their perspectives on a particular phenomenon of interest. In-depth interviews are useful to discover detailed 
information about a person's thoughts and behaviors, or to explore newly identified issues in depth. Interviews are often used to provide context to other data (such as outcome data), offering a more complete picture of what may be happening in quantitative data and why (Cuádraz \& Uttal, 1999). The key feature of the in-depth interview is that it is intended to combine structure with flexibility (Farrell, Murphy, \& Schneider, 2002). It involves conducting intensive individual interviews with a small number of respondents to explore their perspectives (Boyce \& Neale, 2006).

Naturalistic inquiry research uses nonrandom, purposive sampling techniques, where a set of criteria or list of attributes are created to select information-rich cases (Frey, Boton, \& Kreps, 1999). Using these criteria, informants provide unique insight into a culture; emergent design introduces, or changes, procedures during the research process; and member checks occur when informants are given a synopsis of research findings for feedback (Frey, Boton, \& Kreps, 1999). There are several methods of purposeful sampling, including maximum variation sampling, homogenous sampling, typical case sampling, extreme (deviant) sampling, critical case sampling, total population sampling, and expert sampling (Lincoln \& Guba, 1985; Frey, Boton, \& Kreps, 1999).

\section{Overview of Geographic Information Systems}

A geographic information system (GIS) is a system designed to capture, store, manipulate, analyze, manage, and present all types of geographical data (Bleich, et al., 2010). GIS is the "merging of cartography, statistical analysis, and database technology, digitally creating and "manipulates" spatial areas that may be jurisdictional, purpose, or application-oriented" (Hoefer, Hoefer, \& Tobias, 1994).

Neighborhoods form the physical and social environment within which people conduct their daily activities and therefore neighborhood quality has a direct and significant impact on the quality of life of its residents (Talen \& Shah, 2007). One example of the construction and use of a neighborhood GIS is found for the city of Urbana, Illinois as a prototype (Talen \& Shah, 2007). Participants of this study were encouraged to interact with the GIS along with study coordinators, and to respond to several questions about their neighborhood, thus facilitating with the creation of the GIS map (Hoefer, et al., 1994; Talen \& Shah, 2007).

GIS can be used to illustrate location specific data in a visual and convenient format. One study used paper maps to visualize the impact of poverty, education, crime rates, and unemployment for the city of Rockford, IL; the authors remarked that this was a cumbersome task, could have been made more manageable with the use of GIS (Hoefer, et al., 1994). Along with the GIS' technical advantages, GIS is being increasingly used as a policy and decision making tool for community planning (Ammerman, 1997). Efforts to strengthen the link between neighborhood environmental characteristics in Memphis, neighborhood residents' access to health resources, and health outcomes takes GIS beyond its conventional role in planning to develop a 
'neighborhood GIS' map, potentially aiding in the visualization, description, and evaluation of the local environment in relation to the proposal purpose and policy development. Papas et al. (2007) found that proximity may serve as a surrogate measure of influence with respect to the built environment; aspects of the built environment closest to a residence have a stronger influence than those farther away. Connectivity is also a measure that can be gauged through GIS, and that gives a measure of how effectively residents can walk around their neighborhoods (Tresidder, 2005).

Talen and Shah (2007) conducted a study that connected residents' perceptions of the surrounding environment to actual environmental characteristics to build a neighborhood GIS model. This study found that there was a positive response of the residents who participated in the neighborhood GIS exercise in Urbana, and the enthusiasm of the city officials, suggesting that GIS can be effectively used for enhancing community participation and resource utilization. Though the literature is scarce regarding community and neighborhood-level applications, these studies show that exposure to GIS can potentially enlighten both policy makers and community members of resources otherwise unknown, and bring community members into the planning process, facilitating better outcomes.

\section{Problem Statement}

The socioecologic conceptual model describes a hierarchy of influences that affect health outcomes (Northridge, Sclar, \& Biswas, 2003). The hierarchy includes macro-level central influences such as racial/ethnic segregation and education disparities that affect intermediary factors such as land use, transportation systems, and the availability of parks, shopping, recreational, or healthcare facilities. These in turn may impact more proximate factors, such as environmental stressors (crime and safety), health behaviors (nutrition, physical activity), and social integration or support systems. The last level in the hierarchy is at the individual level, where health outcomes such as type 2 diabetes and obesity are ultimately affected by all factors from the higher levels of the socioecologic hierarchy (Mobley, Root, Finkelstein, Khavjou, Farris, \& Will, 2004).

Urban form denotes physical features of neighborhoods, such as suburban sprawl (pockets of homogenous housing and long commutes to inner cities) or inner cities (a mix of dwellings, restaurants, shops, and businesses) (Mobley, Root, Finkelstein, Khavjou, Farris, \& Will, 2004). One study evaluated the hypothesis that that mixed-use neighborhoods have more destinations worth walking (of higher quality) to than do more homogenous neighborhoods, and thus, it follows that populations in such environments may get more exercise, become less obese, and thus better manage the diabetes health state (Mobley, Root, Finkelstein, Khavjou, Farris, \& Will, 2004). This study also suggested that factors of the built environment, such as the availability of fitness centers or healthy foods, may assist in the understanding of lower obesity rates in mixed urban environments (Mobley, Root, Finkelstein, Khavjou, Farris, \& Will, 2004). 
The interaction between women's perceptions of their health; their environment; perceptions of cultural and economic influences; and how these perceptions influence utilization of the environment with regard to physical activity has not be thoroughly explored in the literature. Furthermore, quantitative studies that have attempted to explore relationships between physical activity and perceived/objectively measured proximity to resources have produced inconclusive results. A mixed-methods approach (a combination of individual interviews, and objective quantification of participant neighborhoods) has been selected to guide this proposal for two specific reasons: 1) Nationally representative, quantitative data have not completely accounted for environmental variables influencing lifestyle behaviors, and particularly, obesity disparities and obesity and diabetes management considerations among low-income women. The qualitative component of this study seeks to understand the rationale and motivation of individual behavior, as well as how low-income African American women in these particular environments operate with regard to weight loss. 2) The environment has been implicated as a key issue and is thus being evaluated; supplementing subjective qualitative data with objective environmental data may be useful in stressing environmental areas of improvement. The purpose of this mixed-methods study is to describe perceptions of weight loss experiences in relation to neighborhoods and environment among low-income African American Memphis women with type 2 diabetes.

Just as there are factors relating to individuals that may contribute to or help evaluate diabetes management issues within 38106 and 38109, there are also factors within the community environment that may do the same. These factors could potentially include the availability or lack of services, information, and other support; the degree of accessibility and barriers to, and opportunities for information, services, and other forms of support; the financial, social, and other costs and benefits of change; and such allencompassing factors as poverty, community living conditions, or economic and official policy conditions (Community Tool Box, 2014).

The long-term goal of this study is to gain a deeper understanding of the 38106 and 38109 communities with respect to diabetes, and to provide context for the decisionmaking process of women with type 2 diabetes. Additionally, studies have repeatedly shown that community design has a significant impact on health behaviors, levels of physical activity and social cohesion. Communities have individual needs and assets, as well as an individual social structure and culture (Community Tool Box, 2014).

Attempting to address the poor health outcomes of populations without fully considering the day-to-day context of these individuals, their proximal environment, and decisionmaking rationale can contribute to implementation failure. Failure in recognizing and utilizing community resources not only represents tackling community problems without employing all of the tools available; this represents missing an opportunity to increase communities' capacity to resolve its own difficulties and self-produce change.

Further, exploration of the validity of perceived neighborhood environments among individuals living within the same neighborhoods and how those perceptions influence behavior choices such as physical activity and diet is an important 
consideration in understanding and introducing potential solutions. This study seeks to serve not only to uncover perceptions of participants communities with regard to physical activity, as well as available resources that encourage activity and health maintenance, but to uncover underlying cultural and social structures present to assist in the understanding of how to address the community's needs and utilize its resources. This consideration can be the first step in communities learning to use available resources to solve problems and improve community life.

Weight loss, exercise, and proper nutrition are important considerations in the management of obesity and type 2 diabetes, particularly among women; however, the difficulty that obese individuals have losing weight may be exacerbated by limited economic circumstances and environmental resources. Additionally, two ZIP codes in Memphis, TN (38106 and 38109) have been demonstrated to have substantially higher diabetes hospitalization rates than surrounding areas (CHEER, 2010). These areas also demonstrate high rates poverty and feature a large African American population. As it is postulated that rates of obesity are also high concurrent to high rates of diabetes hospitalizations, an examination of cultural, social, economic, and environmental factors that may lead to inadequate diabetes management, along with an investigation of land-use and factors of the built environment in these two areas is needed. This study was embarked upon with the intention of shedding light on factors that encourage and discourage weight loss and health maintenance, to inform future research directions in similar circumstances and populations, and to inform and identify efficient intervention strategies and potential courses of action that are effective and can be sustained over the long-term.

\section{Study Purpose}

This study serves as a mixed-methods attempt to describe culturally relevant perceptions of the circumstances of southern African American overweight/obese women who have been diagnosed with type 2 diabetes, as well as their perceptions of environmental conditions that may promote or inhibit weight loss efforts. The secondary purpose of this study was to examine specific attributes of the built environments of 38109 and 38106 that may promote or inhibit weight loss, health maintenance, and physical activity efforts.

\section{Research Questions}

This study sought to answer the following research questions:

1. What are methods of weight loss, including forms of environmental utilization, employed by low-income, African American women with type 2 diabetes mellitus in the 38106 and 38109 ZIP codes of Memphis, TN? 
2. What are the sociocultural, economic, and environmental influences that encourage or discourage successful weight loss, health maintenance, and physical activity among low-income, African American women with type 2 diabetes mellitus in the 38106 and 38109 ZIP codes of Memphis, TN?

3. What are objective environmental barriers to achieving weight loss that can be gauged through Geographic Information Systems?

\section{Study Objectives}

This mixed-methods study addressed weight loss and health outcomes among overweight and obese low-income African American women with type 2 diabetes, and more specifically aimed to:

1. Assess methods of weight loss among low-income African American women with type 2 diabetes in the two areas in Memphis, TN, identified as having high rates of diabetes hospitalizations (38109 and 38106) in comparison to surrounding areas.

2. Uncover and describe low-income African American women's perceptions of the specific sociocultural, economic, and environmental factors that influence or prohibit successful weight loss while possessing type 2 diabetes, utilizing individual interviews, who reside in the selected areas of 38109 and 38106.

3. Discover possible objective environmental barriers to achieving weight loss through use of Geographic Information Systems (GIS) in 38109 and 38106, by assessing the proximal environment for mixed-use or homogeneity, examining the availability of area resources, and quantifying walkability to selected resources in the identified areas.

\section{Assumptions}

The design and conduct of this study were based on the following assumptions:

1. The phenomena of interest (weight loss and health maintenance in type diabetes) will be studied in a naturalistic manner; that is, the phenomena is be assumed to occur in its natural context and possesses the ability to be assessed through indepth interviews

2. The phenomena will be examined without preconceived notions or a priori expectations 
3. Because of the interpretive nature of the study, the researcher, while trying to see the situation from the point of view of those being studied, cannot escape from providing a personal interpretation of the situation

4. All information obtained via Geographic Information Systems, including US Census data and the addresses of all locations of interest, are presumed to be accurate and up-to-date

\section{Limitations}

The following limitations were identified prior to study initiation:

1. Purposive samples, regardless of the type of purposive sampling used, can be highly prone to bias

2. The subjectivity and non-probability based nature of unit selection in purposive sampling means that it can be difficult to defend the representativeness of the sample

3. A literature review was conducted prior to study initiation and may also subject the researcher to interpretive bias

4. The interview procedures may subject participants to recall bias, and participants may not be able to readily recall phenomena which directly pertain to the question

5. Imprecise information delivery by study participants may misrepresent the true meaning of material received by the researcher

6. Replication of a similar study may not yield similar results, largely because human behavior and situations are highly changeable.

\section{Organization of the Study}

Chapter 1 introduced the problem, weight loss among type 2 diabetic women who are low-income, provides background information on the study areas and the context of the study, details an overview of the methodological philosophy, and identifies the study purpose, research objectives, a priori assumptions and limitations. The chapter describes how difficulties in weight loss may stem from environmental concerns, exacerbated by being low-income and possessing type 2 diabetes. Chapter 2 provides a detailed description of the context of study and includes a review of the literature related to the major and related concepts in the conceptual framework. Chapter 3 describes the methods used in the study including the research design, participant identification, data collection instruments and procedures, Geographic Information Systems procedures, and data analysis techniques. Chapter 4 presents the analysis of study findings. Chapter 5 
discuses and interprets findings, draws conclusions, and makes recommendations to inform policy and future research.

\section{Glossary of Terms}

\section{Active transportation}

\section{Built environment}

Community

\section{Connectivity}

\section{Health}

Land use

Land-use mix

Mixed-use

Physical activity

Proximal environment

Resource
Any form of human-powered transportation, including walking

Comprises urban design, land use, and the transportation system, and encompasses patterns of human activity within the physical environment

Synonymous with ZIP Code and ZCTA, this term includes the residential area and constitutes housing, schools, recreation, socialization and open space areas. Ideally, convenient access to public transportation, options for safe travel, and resources are provided to sustain individual living are included within this context

The directness of travel to destinations

Refers to physical, mental, and social well-being

The distribution of activities and resources across space, including the location and density of different activities, where activities are grouped into relatively course categories such as residential, commercial, office, industrial, and others

Diversity or variety of land uses

Development that combines two or more of the types of development: residential, commercial, office, industrial, or institutional

Any body movement that results in energy expenditure and can include both leisure and nonleisure physical activity

Refers to the areas closest to a point of reference

An identified business, area, space, or entity that can be utilized to contribute to the well-being of an individual or population 
Walkability

\section{ZIP Code}

ZCTA
The extent to which the built environment is friendly to the presence of people living, shopping, visiting, enjoying, or spending time in the area. Factors affecting walkability include, but are not limited to: land use mix; street connectivity, residential density (residential units per area of residential use); places to go near residences. Major infrastructural factors include access to mass transit, present and quality sidewalks, buffers to moving traffic, pedestrian crossings, aesthetics, nearby local destinations, air quality, shade or sun in appropriate seasons, and traffic volume and speed. The diversity of people, especially the presence of children, seniors, and people with disabilities, denotes the quality, completeness, and wholesomeness of a walkable space.

Synonymous with community and ZCTA

ZIP code tabulation area; this term represents a statistical entity developed by the United States Census Bureau for tabulating summary statistics, and is a generalized area representation of United States Postal Service ZIP code service areas; for the purposes of this examination, the term will be synonymous with ZIP code and community 


\section{CHAPTER 2. LITERATURE REVIEW}

This literature review will be presented in three parts. The first section will discuss pertinent literature that includes the following topics: obesity, type 2 diabetes, and economic considerations; obesity and associated comorbidities with emphasis on type 2 diabetes; type 2 diabetes and its association with African Americans, women, and poverty; and weight loss implications among diabetics in the literature. The second section will provide an overview of physical activity and obesity outcomes, and the associations between physical activity, environment, community design, and health outcomes in the literature. The final and third section will discuss the socioecological model and its applications.

All initial literature searches were conducted in 2012 using PubMed (National Center for Biotechnology Information et al.) and Science Direct (2012). Later literature searches conducted in March 2014 also utilized Scopus (Elselvier Science Publishers, 2004) to identify new literature pertaining to the topics of interest.

\section{Part 1: Obesity Considerations}

\section{Obesity and type 2 diabetes demographic information and economic considerations}

Obesity has been recognized as a growing epidemiological, societal, and economic problem in the United States. According to the CDC, during the past 20 years there has been a dramatic increase in the prevalence of obesity in the United States (Centers for Disease Control and Prevention, 2013). Thirty-three states have a prevalence equal to or greater than 25\%; nine of these states (Alabama, Arkansas, Kentucky, Louisiana, Mississippi, Missouri, Oklahoma, Tennessee, and West Virginia) have prevalence of obesity equal to or greater than $30 \%$. Obesity in adults is defined as a body mass index (BMI) greater than $30 \mathrm{~kg} / \mathrm{m}^{2}$ (Seidell, 1995a, 1995b). Obesity has reached dramatic proportions in the United States, with young adults having a particularly higher risk (Karnehed, Rasmussen, \& Kark, 2007; Pereira et al., 2002; Whitaker, Wright, Pepe, Seidel, \& Dietz, 1997). Some studies suggest that young adulthood is associated with an average weight gain of 1-2 pounds per year, with the largest gains occurring during the early to mid-twenties. Data also indicate that more than half of the individuals between the age of 20 and 39 years are already overweight or obese (Clarke, O'Malley, Johnston, Schulenberg, \& Lantz, 2009; Karnehed, et al., 2007). Similarly, the obesity epidemic is rapidly becoming a worldwide problem as well (Cecchini, Sassi, Lauer, Lee, GuajardoBarron, \& Chisholm, 2010). Rates have doubled or tripled in many countries, with Mexico's population being one of the most affected (Cecchini, Sassi, Lauer, Lee, Guajardo-Barron, \& Chisholm, 2010). Additionally, overweight and obesity are now the prominent features of malnutrition in South Africa, where a third of women and a tenth of men are obese, with highest rates in black women and white men. Referencing political and economic transition, rates of obesity have also risen in Russia, where now, one in 
four women and one in ten men are obese; rates are projected to grow rapidly in the coming years (Cecchini, Sassi, Lauer, Lee, Guajardo-Barron, \& Chisholm, 2010).

Obese individuals are more likely to develop several chronic conditions, including diabetes, cardiovascular disease, hypertension, and various forms of cancer when compared to nonobese counterparts (Dixon, 2010). Additionally, obesity is also associated with impaired quality of life, psychosocial disturbance, limitations in socioeconomic success, and limited access to quality care. It is estimated that there are more than 300,000 annual deaths attributed to overweight and obesity in the USA and $80 \%$ of these are in subjects with a BMI greater than 30 (Allison, Fontaine, Manson, Stevens, \& VanItallie, 1999; Dixon, 2010; Finkelstein, Fiebelkorn, \& Wang, 2004; Must et al., 1999).

The economic burden of obesity has been and continues to be a significant strain to the United States healthcare system. In 1995 it was estimated that overweight, obesity, and inactivity generated $9.4 \%$ of direct health costs in the US (Dixon, 2010). In 2000, the total financial burden of obesity on the United States was estimated to be around $\$ 117$ billion, accounting for up to $7.0 \%$ of national health expenditures (Finkelstein, et al., 2004; Must, et al., 1999; Thompson \& Wolf, 2001). Estimates of the average per-person annual health care costs for obesity-related conditions compared with people who have normal weight have ranged from \$395 to \$732 in the literature (Allison, Zannolli, \& Narayan, 1999; Finkelstein, Fiebelkorn, \& Wang, 2003).

Startling projection models show that by the year 2030, approximately $90 \%$ (86.3\%) of all American adults would become overweight or obese and $51.1 \%$ of them would be obese (Wang, Beydoun, Liang, Caballero, \& Kumanyika, 2008). By 2018, all states are projected to have a midpoint of no less than $\$ 1,000$ spent on obesity per capita with the exceptions of Colorado and the District of Columbia. Black women (combined prevalence 96.9\%) and Mexican American men (91.1\%) would be the groups most affected. In children and adolescents, prevalence of overweight would increase 1.6-fold (to $\sim 30 \%$ ) by 2030 . Mexican American young boys and black adolescent girls would have the highest prevalence (both $41.1 \%$ ), a level that would be 10 percentage points higher than the national average. Further, the prevalence in MA adolescents will increase by twofold and among African American teens, by 1.8-fold, the largest increases (Wang, et al., 2008). Health officials, scientists and the public should be increasingly concerned about the obesity epidemic, its rates of progression and prevalence, and the accompanying morbidity, mortality, health care costs, and loss of quality of life.

In 2010, type 2 diabetes accounted for more than $95 \%$ of the 25 million patients with diabetes in the US, and an additional 79 million individuals were estimated to be pre-diabetic (National Diabetes Statistics, 2011; Diabetes, 2011). In 2007, the annual economic burden of diabetes was estimated at \$174 billion, with $\$ 116$ billion in excess medical expenditures and $\$ 58$ billion in reduced productivity (National Diabetes Statistics, 2011). Diabetes also takes a toll on the health care system, as 1 in 10 health care dollars is attributed to it (Diabetes, 2011). Average medical expenses for patients with diabetes are nearly twice as high as those for patients who do not have diabetes. 
Additionally, type 2 diabetes is more prevalent in minority races and ethnicities, as evidenced by Hispanics having a $66 \%$ greater risk, and non-Hispanic blacks having a $77 \%$ greater risk for developing it than non-Hispanic whites (National diabetes Statistics, 2011).

The rates of obesity and type 2 diabetes in the United States tend to follow a socioeconomic gradient, such that the burden of disease falls disproportionately on people with limited resources, racial-ethnic minorities, and the poor (Clarke, et al., 2009; McLaren, 2007; Mobley et al., 2006). As shown in Table 2-1, obesity prevalence among any stratified adult group is highest among African American women.

Also among women, higher obesity rates tend to be associated with low incomes and low education levels (Mobley, et al., 2006). There has been a consistently inverse association with respect to obesity for women in developed societies, where obesity exhibits a higher likelihood of occurrence among women in lower socioeconomic strata. In contrast, the relationship for men and children in developed societies has historically been inconsistent (McLaren, 2007), but in developing societies, there is a strong direct relationship that has been observed for women, men, and children, with a higher likelihood of obesity among persons in higher socioeconomic strata (McLaren, 2007). Because of the increasing prevalence of obesity in several countries, including the United States, and well documented disparities in the use of health care, continued monitoring of the socioeconomic patterning of weight is important.

\section{Obesity, type 2 diabetes, and hypertension}

Type 2 diabetes has historically been strongly associated with obesity, both causally and by sharing common etiological factors in susceptible individuals (Lean, Powrie, Anderson, \& Garthwaite, 1990). It is also frequently suggested that obesity contributes to the accelerated ischemic vascular disease and mortality of patients with type 2 diabetes; therefore, weight loss is heavily advocated for obese diabetic individuals. One study estimates that $80 \%$ of type 2 diabetes cases can be attributed to obesity (Cornier et al., 2005); another study places this estimate closer to between $60 \%$ and $90 \%$ of occurrences (Rana, Li, Manson, \& Hu, 2007). Obesity in patients with diabetes is also associated with increased morbidity and mortality (Cornier et al., 2005). Increasing obesity in patients with diabetes is associated with increased prevalence of other cardiovascular risk factors and adverse cardiovascular outcomes. Some studies have indicated high effectiveness of intentional weight loss in reducing dependence on diabetes, hypertension, and lipid-lowering medications (F. X. Pi-Sunyer, 2005), however, the prevalence of diabetes in the United States continues to reach epidemic proportions (Cornier, et al., 2005). An additional, commonly associated comorbidity of obesity includes hypertension. There also appears to be an added effect of race on the obesityhypertension relationship (Burt et al., 1995). African Americans (non-Hispanic blacks) have the highest prevalence of hypertension across gender and age, with the prevalence of hypertension in Mexican Americans somewhat less than Caucasians (non-Hispanic Caucasians). 
Table 2-1. Table depicting adult obesity rates by ethnicity - NHANES 2011-2012.

\begin{tabular}{lcc}
\hline Ethnicity & Women \% & Men \% \\
\hline Non-Hispanic white & 32.8 & 32.4 \\
African American & 56.6 & 37.1 \\
Hispanic & 41.4 & 40.1 \\
\hline
\end{tabular}

Reprinted with permission. Source: Ogden C. L., Carroll, M. D., Kit, B.K., \& Flegal K. M. (2014). Prevalence of childhood and adult obesity in the United States, 2011-2012. Journal of the American Medical Association, 311(8), 806-814. 
The prevalence of hypertension increases with age in all three racial groups, and in all three racial groups there is a gender difference in hypertension rates. In the younger age strata, the rates of hypertension are greater in men compared with women (Burt, et al., 1995). With increasing age, the gender difference in prevalence disappears after 60 years of age for Mexican Americans and Caucasians. For African Americans, however, the prevalence of hypertension is equivalent in men and women by age 50-59 and becomes higher in African American women compared with African American men (Burt, et al., 1995). The extent to which a greater prevalence and degree of obesity explains the higher prevalence of hypertension in older African American women has not been determined. With respect to metabolic syndrome and cholesterol level, compared with Caucasians, HDL cholesterol levels are higher in African Americans (Ford, Giles, \& Dietz, 2002). The higher HDL cholesterol levels among African Americans cannot be explained by racial differences in BMI or other factors that have an effect on plasma HDL cholesterol concentration, such as alcohol intake, physical activity, or smoking (Ford, Giles, \& Dietz, 2002).

Colditz and colleagues (Colditz, Willett, Rotnitzky, \& Manson, 1995) from the Nurses' Health Study assessed the influence of three different factors on risk for developing diabetes in women: (1) BMI at age 18 years, an assessment of adolescent obesity, (2) weight gain after age 18, and (3) attained BMI at 30 to 55 years of age, assessing adolescent obesity as well as weight gain. The age-adjusted risk for developing diabetes over 14 years of age increased from a standardized risk of 1.0 for a BMI of 22 at age 18 to a relative risk (RR) of 93.2 for an attained BMI of 35 at 30 to 55 years of age (Colditz, et al., 1995)," Egede and Dagogo-Jack (Egede \& Dagogo-Jack, 2005) list additional risk factors for type 2 diabetes as being 45 years of age, overweight, possessing a family history of diabetes, habitual physical inactivity, and being a member of a racial minority group. In addition, women who have polycystic ovary syndrome also possess a higher likelihood of developing type 2 diabetes (Dagogo-Jack, 2003).

To date, routine interventions aimed at weight loss in patients with type 2 diabetes have not been particularly effective. Although it is possible for patients with diabetes to successfully lose weight, it appears to be more difficult for them, especially when longterm success is measured (Cornier, et al., 2005). Pi-Sunyer (F. X. Pi-Sunyer, 2005) suggests that reasons for this include negative biological responses to weight loss, psychological fatigue from a long history of weight loss intervention attempts that have failed, and weight gain with pharmacotherapy. Persons with limited resources may have an even more difficult time losing weight needed to effectively manage their diabetes.

\section{Obesity, type 2 diabetes, African American women in the context of poverty, and sociocultural implications}

Efforts to explain the disparity in obesity prevalence among women have generally focused on individual-level factors, including sociodemographic characteristics, health status, and lifestyle and behavior measures (Bleich, et al., 2010). These factors, however, only partially explain measured obesity disparities. Sociocultural factors, 
particularly with regard to type 2 diabetes, has been very minimally explored in the literature. Cultural determinants can shape health behaviors and serve as a lens for perceiving and interpreting experiences (BeLue et al., 2009). Further, culture is the dynamic and evolving socially constructed reality that exists in the minds of social group members. It is the shared set of values, ideas, concepts, and rules of behavior that allow a social group to function and perpetuate itself (Hudelson, 2004). The combination of social and cultural influences that produce individual perception of reality is a powerful consideration. One significant cultural influence within the African American community that has been highlighted in the literature is dietary behavior (Kumanyika et al., 1996). In this study, focus group interviews were used to explore cultural aspects of eating patterns among low- and middle-income African Americans recruited from an urban community in Pennsylvania. Participants identified eating practices commonly attributed to African Americans and felt that these were largely independent of socioeconomic status. Although participants were uncertain about links between African American eating patterns and African origins, they were clear about influences of slavery and economic disadvantage. An acknowledgement of sociocultural values within the African American community lends insight into an understanding of type 2 diabetes risk within these communities, as well as a more full perception of minority health disparities overall.

Additionally, Bleich et al examined whether differences in race disparities existed among black and white women living in the same social context and found that disparities in obesity are eliminated when comparing black women and white women in their specific dataset, which examined persons living in low-income racially integrated neighborhoods and with similar socioeconomic means (Bleich, et al., 2010). This study showed that racial segregation is not the primary driving factor in health disparities among black and white women; rather, economic means and environmental characteristics may play a larger role in predicting obesity risk than race. Racially segregated neighborhoods tend to have more limited resources when compared to more racially integrated neighborhoods. This also is consistent with a growing body of research relating obesity prevalence to the built environment (Papas et al., 2007).

Another study examined relationships between the built environment and cardiac risk or obesity in a population of low-income women of all races/ethnicities and varied education levels, and found that independent effects were found of built environment measures and other socioecologic characteristics such as crime rate, area affluence, income inequality, and racial/ethnic segregation (Mobley, et al., 2006). Poorer neighborhoods tend to be more densely populated by fast-food restaurants, and limited availability of fitness facilities and outdoor recreation (Ball, Mishra, \& Crawford, 2002; Mobley, et al., 2006).

Several studies have referenced the varied impact of obesity, hypertension, and diabetes in African Americans when compared to other races and ethnicities (Douglas et al., 2003; Stevens, 2000). Relative rates of type 2 diabetes are also greater in African Americans compared with Caucasians. Brancati et al (Brancati, Kao, Folsom, Watson, \& Szklo, 2000) reported that African American women were more than twice as likely, and 
African American men were 1.5 times as likely to develop type 2 diabetes than their Caucasian counterparts. This association remained statistically significant even after adjustment for age, BMI, waist to hip ratio and other risk factors, indicating that obesity alone could not account for the entire race disparity in prevalence of type 2 diabetes. Studies have suggested that many of the estimated $30.5 \%$ of all adults, and $54.8 \%$ of adults with diabetes, who have a body mass index (BMI) of 30 or greater do not have a diagnosis of obesity. The records of 265 people with diabetes in the Racial and Ethnic Approaches to Community Health (REACH) 2010 Charleston community were audited for race, sex, BMI, and a documentation of obesity, to determine the likelihood of a diagnosis of obesity for people with BMI of 30 or greater, based on race and sex (Racial and Ethnic Approaches to Community Health, 2012). Significant differences in diagnosis were observed by race, with three times as many records of obese White people with diabetes containing a diagnosis of obesity as of diabetic African Americans. Disparities in prevalence of obesity based on a BMI of 30 or greater were observed as well, with a higher proportion of African Americans meeting the criteria. Studies suggest that these disparities may contribute to the increased burden of disease experienced by African Americans with diabetes (Jenkins et al., 2004). Dagogo-Jack (Dagogo-Jack, 2003) published an article evaluating disparities in type 2 diabetes as well as implications for its prevention and management, and found that there may be a number of reasons why ethnic disparities exist, which include genetic and environmental factors. However, when diagnosed, affected persons, regardless of ethnicity, respond well to modified dietary approaches, pharmacotherapy, and aggressive diabetes management protocol (DagogoJack, 2003). Researchers further report that racial differences in potentially modifiable risk factors, particularly adiposity, accounted for $47.8 \%$ of the excess risk in African American women (Hedley, et al., 2004).

As noted before, the literature suggests that African American women of low socioeconomic means are most vulnerable group to becoming obese and developing type 2 diabetes. Several studies suggest that this may be due to systematic delivery of suboptimal care. Another posited correlation is due to prices of foods and incomes, which may affect food choices, dietary habits, and diet quality (Duncan, et al., 2011). Food insecurity and obesity are also linked, with food insecurity being defined as "limited or uncertain availability of nutritionally acceptable or safe foods"; women reporting food insufficiency typically have lower quality diets (Drewnowski \& Specter, 2004). A behavioral model has been confirmed in which household members faced with diminishing incomes first consumed less expensive foods to main energy intakes at a lower cost. The resulting low-cost of energy-dense foods may in turn promote overconsumption of such foods (Drewnowski \& Specter, 2004). This study also indicated that women in food-insufficient households consume the same amount of energy as did women in food-sufficient households (1959 compared with $1868 \mathrm{kcal} / \mathrm{d}$ ).

Other studies suggest that among some low-income women, there may psychosocial and familial factors that play a role in obesity development. A crosssectional study that examined African American women in ambulatory care facilities suggested that contributing factors to obesity incidence among this group include previous childhood obesity; overweight parents and siblings; and higher parity, compared 
to normal and overweight women. Further contributing factors include having sedentary lifestyles, higher caloric intake, earlier menarche, and earlier age of first childbirth (Rowe, 2010). In addition, nutrition programs designed in the past were primarily intended to help avert nutritional inadequacies. However, there has been evidence to suggest that Food Stamp Program (FSP) participation and overweight may also be associated with obesity among low-income women, potentially due the majority of FSP resources being spent on less expensive, more energy dense, high calorie foods to conserve FSP resources for longer periods of time (Frongillo, 2003). Furthermore, controlled clinical trials revealed that African American women's risk factors for obesity were associated with a clustering of metabolic disorders (i.e., non-insulin-dependent diabetes mellitus, hypertension, lipid abnormalities, atherosclerotic cardiovascular disease (Rowe, 2010). In this study there were also significant associations of African American obese women having higher anxiety levels, poorer perceptions of physical health and more psychosocial problems in the family of origin than the normal or overweight women (Davis, Rovi, \& Johnson, 2005).

With regard to the implications of barriers perceived faced by women in terms of physical activity and healthy eating, it is suggested that these may vary in young women according to their social and personal circumstances (Andajani-Sutjahjo, Ball, Warren, Inglis, \& Crawford, 2004). Influences including as having children, lower socioeconomic status, lack of access to good quality, low-cost foods (Havas, Treiman, Langenberg, et al., 1998), limited knowledge (Lopez-Axpiazu, Martinez-Gonzales, Kearney, Dibney, \& Martinez, 1999) and being overweight itself are demonstrated to be likely to impact women's ability to adopt healthy habits. Andajani-Sutjahjo, Ball, Warren, Inglis, \& Crawford (2004) conducted a quantitative study that assessed the perceived barriers to physical activity and found that motivation, time, cost comprised the majority of the responses. Perceived barriers to healthy eating included lack of motivation, lack of time due to work, and cost of food items (Andajani-Sutjahjo, Ball, Warren, Inglis, \& Crawford, 2004). Normal weight individuals have been found to engage in significantly more physical activity than overweight individuals, who engage in) more physical activity than obese individuals (Blanchard, McGannon, Spence, Rhodes, Nehl, Baker, \& Bostwick, 2005). Additionally, the presence support partners when attempting to lose weight has also shown to be of benefit to obesity treatment (Gorin, Phelan, Tate, Sherwood, Jeffery, \& Wing, 2005) demonstrating the efficacy of social support.

Studies on gender-related and race-related disparities of ideal body weight and perception of body weight are prevalent (Carr \& Friedman, 2005; Connor-Greene, 1988; Faith, Flint, Fairburn, Goodwin, \& Allison, 2001; Johnston, Reilly, \& Kremer, 2004; Muth \& Cash, 1997; Pliner, Chaiken, \& Flett, 1990). Estimates indicate that more than $35 \%$ of adult women in the United States are overweight (Hedley et al., 2004). Of that figure, $48 \%$ of African American women between the ages of 25 and 49 are overweight, compared with 26\% of European American women (Hedley, et al., 2004). Further disparities exist between women of various cultural backgrounds with respect to perception of body image and ideals, suggesting that African American women are less likely to perceive obesity as a problem. Furthermore, African American women are also less likely to recognize that they are overweight or obese (Duncan et al., 2011; Mama et 
al.,2011; Sivalingam et al., 2011). Like the high rates of obese and overweight found in African American women, an increased likelihood of being exposed to overweight mothers and significant other adults as children has been postulated to encourage a higher tolerance for obesity in the African American community (Wilson, Saargent,\& Dias, 1994; Gipson et al., 2005). In one study of African American and White women's experiences with weight loss methods, many African American women recalled being pressured by their families to accept being overweight (Davis et al, 2005, 1540). With a higher tolerance for heavier weight found among families and social communities in African Americans, ranges of healthy body images can therefore be influenced to accept larger and sometimes unhealthy body sizes (Kronenfield et al., 2010). An additional study demonstrated that among severely overweight African American women who had tried to lose weight, only $36 \%$ reported that their husband or boyfriend thought that they were overweight. In the same study, when asked if being overweight or obese had ever caused them difficulty in personal or family relationships, or getting a job, $81 \%$ and $96 \%$ respectively of the women responded "not to my knowledge". (Kumanyika et al., 1993).

Even with this research, studies have also established that cultural definitions of weight perception and weight related health complications are not so simple. Although several studies have reported that heavier weight is more highly tolerated within the African American community, another study suggested that many African American women recognized that they were obese and overweight, and knew of their weight related health complications (Stevens, Kumanyika, \& Keil, 1994). However, even though heavier weights were identified as increasing risk for health complications, many of the women still reported being satisfied with their weights (Stevens, Kumanyika, \& Keil, 1994; Neff, 1997; Lovejoy, 2001). Similarly, another study showed that in a group of African American adolescent girls, many reported that their ideal weight or body size was influenced more by self-satisfaction then actual body size. Body weight, for them, had more do with being comfortable with your weight than being of an "acceptable" size (Boyington, 2008). Body weight satisfaction and weight loss for African American women, it has been said, has more to do with subjective cues including sexual attractiveness, how clothes fit and how they view themselves in the mirror rather than health reasons alone (Melynk \&Weinstein, 1994). Further, Melnyk and Weinstein suggest that African American women may place less value on the cosmetic aspects of body weight, compared to White women, as their family and social roles change during their lifetimes.

The Thrifty Gene Theory states that non-European minorities, such as African Americans, have survivor genes that allow for food conservation and energy storage during natural disasters and famines or during food shortage. They hypothesize that the modern food rich environment may set off the genes, increasing the risk for obesity and type 2 diabetes (Egede \& Dagogo-Jack, 2006). Though this is a plausible theory, another study suggests that minority health outcomes have been demonstrated to enhance preconceptions of genetically based racial inferiority views and lead to generalizations that can have a negative effect on understanding and addressing the health disparity found in African American health outcomes (Darity Jr. et al., 2010,). 


\section{Weight loss outcomes in type 2 diabetes}

Several studies have explored the hypothesis that weight loss improves survival in obese type 2 diabetes patients. One study indicated that each $1 \mathrm{~kg}$ weight loss over the first year from diagnosis was associated with about 3-4 months increased survival (Lean, et al., 1990). One behavioral faith-based diabetes prevention program study found that using screenings of church participants, there was a high possibility that subjects would achieve a clinically significant degree of weight loss (Dodani \& Fields, 2010). Another study conducted in 2007 found that obesity and physical inactivity independently contribute to the development of type 2 diabetes; however, the magnitude of risk contributed by obesity is much greater than that imparted by lack of physical activity (Colditz, et al., 1995).

Additionally, several studies report that modest changes in lifestyle can protect high-risk individuals from developing diabetes (Knowler et al., 2002; Pan et al., 1997; Tuomilehto et al., 2001). Other studies indicate that weight loss and increased physical activity does seem to slow the development of type 2 diabetes in high-risk individuals. Restriction of fat intake augmented with complex carbohydrate and dietary fiber, is shown to enhance insulin sensitivity (Egede \& Dagogo-Jack, 2005), and has been shown to decrease hemoglobin $\mathrm{A}_{1 \mathrm{c}}$ levels, as well as the need for insulin (Egede \& Dagogo-Jack, 2005). The Finnish Diabetes Study (Tuomilehto, et al., 2001) demonstrated that the risk for developing diabetes was reduced by approximately $58 \%$ over a six-year period through an intervention encouraging weight loss and increased physical activity. Although Finland is not necessarily categorized as "high risk" by U.S. standards, persons with diabetes, regardless of ethnicity, respond well to interventions such as modified dietary approaches, pharmacotherapy, and aggressive diabetes management protocol (Dagogo-Jack, 2003).

Challenges in weight management for diabetic individuals have encouraged studies that explored utilizing total fasting or very-low-energy diets (VLED) over short periods of time. One study found a significant correlation between glucose change and weight loss (Ritchie \& Spencer, 1994). A 10-kg weight loss over 12 weeks was shown to decrease fasting plasma glucose values by $25 \%$. Wing and colleagues (Green \& Thorogood, 2004) also noted that greater weight loss was correlated with greater improvement in glycemia. Persons with greater weight loss had greater improvements in all their risk factors than persons with lesser weight loss (Green \& Thorogood, 2004). Greater amounts of weight lost in obese diabetic individuals is also associated with significant increases in HDL-cholesterol levels (Green \& Thorogood, 2004).

Obesity is difficult to manage in type 2 diabetic and non-diabetic individuals. Diabetic individuals tend to have more difficulty losing weight than non-diabetic individuals because of factors including genetic or metabolic differences, fear of hypoglycemia, anti-diabetes medications, other medications, limited physical activity or diet fatigue (Tuomilehto, et al., 2001). Perceived dietary restrictions, such as limitation of carbohydrate intake, may limit therapeutic choices for diabetic individuals. Health care professionals may be reluctant to recommend meal replacements, widely used by non- 
diabetic individuals. Additionally, some pharmacologic used to treat obesity are relatively contraindicated in diabetic individuals because of their cardiovascular effects (Rabjee, 2004). Weight loss recommendations for non-diabetic individuals have not been clearly translated into guidelines for diabetic individuals; furthermore, the barriers to weight loss among persons of lower socioeconomic status may further limit options and exacerbate difficulty. Expert panels have not yet come to an agreement regarding ideal weight ranges for diabetic individuals; all of these factors contribute to lack of strong directives to treat obesity in persons with diabetes. The Evidence Report for Treatment of Obesity in Adults outlines these approaches to treatment of obesity: dietary therapy, physical activity, behavioral therapy, combined therapy (to include the first three modalities), pharmacotherapy and surgery. Individuals with diabetes typically receive nutrition counseling from a dietitian or nutrition counselor. Many are instructed in low-energydiets (LEDs) and encouraged to increase their physical activity. These diets are moderately successful for non-diabetic individuals with weight losses at one year averaging about $7.2 \%$ of initial body weight (X. Pi-Sunyer et al., 2007). However, less information is available for weight ranges after one year for diabetic individuals. Weight losses in this range offer significant benefits-i.e., reductions in fasting plasma glucose values of $25 \%$ and significant reductions in serum triglycerides - but they often do not lead to "desirable" or non-obese weights. According to the Look AHEAD Trial (X. PiSunyer, et al., 2007), optimal cardiovascular-protective effects appear related to achieving non-obese weights.

With regard to factors that impact weight loss and weight management among overweight and obese African American women, the literature finds that social support is an important component of successful lifestyle-focused weight management interventions (Jeffery et al., 2000; Wolfe, 2004; Young, Gittelsohn, Charleston, Felix-Aaron, \& Appel, 2001). Other factors that may promote long term weight loss for African American women include a change of eating patterns to including eating more fruits and vegetables, changing food preparation methods (broiling and baking instead of frying), watching portion sizes, and reducing red meat consumption. Sanderson, Littleton, and Pulley (2002) identified barriers to physical activity among African American women to include the lack of convenient opportunities for exercise, motivation, perceived health, and environmental factors; Gans and colleagues (2003) add the element of hair care as an important barrier to physical activity in African American women. Further, Wilcox and colleagues (2003) found that education, marital status, self-efficacy, perceived stress, social support, and perceived neighborhood safety were positively associated with physical activity; age, depressive symptoms, environmental factors, quality and health provider interaction and traffic were associated negatively with physical activity among African American women. Physical activity and diet play a significant role in regulating and preventing risk for type 2 diabetes. Research shows that African Americans do want to participate in preventative health behaviors however and report having tried to lose weight in the past (Davis, et al., 2005).

Several studies have examined and developed culturally tailored weight loss interventions specifically for African American women (Banks-Wallace \& Conn, 2002; Bopp et al., 2006; Bronner \& Boyington, 2002; Taylor, Baranowski, \& Young, 1998; 
Wilbur, Michaels Miller, Chandler, \& McDevitt, 2003; Young \& Stewart, 2006). More advanced proposed strategies to further the successful implementation of programs that facilitate weight loss have included more rigorous experimental designs, theoretically based interventions, and validated assessment instruments to detect physical activity change. However, these proposed strategies may be expensive to implement, and even where available, may be poorly used by those for whom attendance at classes is inconvenient or infeasible.

Weight loss perceptions and methods among obese type 2 diabetic women, especially among those who are African American, has been demonstrated ineffective to extremely modest at best, due to complex interactions between numbers of factors. Because studies that have evaluated such interventions typically occur over very short periods of time, there are minimal amounts of research that have examined the effectiveness of such programs longitudinally. Additionally, the impact of historical health disparities and economic and environmental inequities in communities on weight loss efforts has only been minimally explored. Uncovering descriptions of culturally specific practices that prevent or inhibit sustaining behavioral changes, motivation, and weight loss among primarily low-income African American women, particularly within the context of having type 2 diabetes, may provide insight into processes that encourage the maintenance of health among this group.

\section{Part 2: Physical Activity, Obesity and Considerations of Environmental Circumstance}

\section{Physical activity and obesity}

Physical activity has been considered as an important modifiable risk factor for obesity in the public health literature (Fogelholm \& Kukkonen-Harjula, 2000; PHAC and CIHI, 2011), but the empirical evidence on the relationship between changes in physical activity and changes in obesity is mixed in the existing literature. On one hand, minor increases in physical activity and sustained weight losses of $5 \%$ of initial body weight have been shown to reduce risk for developing diabetes by $58 \%$.(Prochaska \& Velicer, 1997). Intensive weight loss programs may facilitate weight loss of $7-10 \%$ in obese diabetes individuals, as well as the reduction of fasting plasma glucose values by $25 \%$ with improvement in lipoprotein and blood pressure levels. However, the public health literature also suggests a negative association between some measures of physical activity and obesity based on cross-sectional studies (Fogelholm \& Kukkonen-Harjula, 2000; PHAC and CIHI, 2011; Fox \& Hillsdon, 2007). Review of the literature reveals that a higher level of causal inference comes from studies where activity and weight change are measured in individuals rather than populations (Wareham, 2007). A systematic review conducted in 2000 describing data from observational cohort studies on physical activity and weight gain in adults concluded that there was inconsistent evidence of the predictive effect of baseline physical activity on subsequent weight gain (Fogelholm \& Kukkonnen-Harjula, 2000). However, they observed that the association 
between weight gain and change in activity or activity at follow-up was stronger, although still modest. The authors suggest that these results can potentially interpreted in three ways:

1. Physical activity is an important factor in preventing weight gain but the true association is not detectable because of measurement error;

2. Less weight gain leads to better exercise adherence - a reverse causality argument;

3. The self-reported physical activity may be a proxy for a general healthy lifestyle a confounding argument (Fogelholm \& Kukkonnen-Harjula, 2000; Wareham, 2007)

Several observational studies on physical activity and weight gain in adults have been performed in the literature. Schmitz, Jacobs, Leon, Schreiner, \& Sternfeld found that changes in physical activity were inversely associated with changes in body weight, and also found that increases in physical activity was associated with attenuation of weight gain. Several studies have generated evidence supporting the benefits of physical activity in slowing weight gain as well (Ball, Brown, \& Crawford, 2002; Hu, Li, Colditz, Willett, \& Manson, 2003; Schmitz, Jacobs, Leon, Schreiner, \& Sternfeld, 2000; Sherwood, Jeffrey, French, Hannan, \& Murray, 2000; Wenche, Kruger, Holmen, \& Midthiell, 2004).

Physical inactivity is associated with increased risk of overall mortality and several common diseases and disorders, including coronary heart disease, stroke, diabetes, some cancers and some mental health problems such as depression (Wareham, 2007). Even in the case of serious overweight and obesity, physical activity substantially reduces disease risk with a potential saving of lives and healthcare costs (Haskell, Lee, Pate, Powell, \& Blair, 2007). Additionally, it is possible to be overweight and also physically fit at the same time, and this reduces risk.

\section{The connection between physical activity and environment}

Several studies have highlighted that environmental factors, broadly defined, may have played a key role in rising obesity rates (Black \& Macinko, 2008; Booth et al., 2005; Feng et al., 2010; Papas et al., 2007). Urban sprawl, defined as the expansion of human populations away from central urban areas into previously remote and rural areas, resulting in low-density communities reliant upon heavy automobile usage, has been implicated in rising obesity in several studies; additionally, it has been linked to physical inactivity, lack of social capital, chronic disease, and private-vehicle commute distances and times (Cho et al., 2006; Doyle et al., 2006; Ewing et al., 2003; James et al., 2013; Joshua et al., 2008; Kelly-Schwartz et al., 2004, Kim et al., 2006; Kostova, 2011; Lee et al., 2009; Nguyen, 2010; Plantinga \& Bernell, 2007; Sturm \& Cohen, 2004). Literature suggests that residents of active communities that promote exercise and activity are 
generally healthier; two key elements of such environments are walkability and safety (Doyle, Kelly-Schwartz, Schlossberg, \& Stockard, 2007). One study examined data from the National Health and Nutrition Examination Survey 2I, 1988-1994 and using a multilevel analysis, found that individuals who live in counties that are more walkable and have lower crime rates tended to walk more and to have lower body mass indices (BMIs) than people in less walkable and more crime-prone areas, even after controlling for a variety of individual variables related to health (Doyle, Kelly-Schwartz, Schlossberg, \& Stockard, 2007). In this same study, among lifelong residents of an area, decreased walkability and more crime were also associated with respondents reporting weight-related chronic illness and lower ratings of their own health (Doyle, KellySchwartz, Schlossberg, \& Stockard, 2007). Additionally, it was demonstrated that the effect of high crime rates was substantially stronger for women than for men, and taking this interaction into account eliminated gender differences in walking, BMI, weightrelated chronic conditions, and self-reported poor health.

African Americans in studies have been found to be less likely to engage in physical activity as a means for type 2 diabetes prevention. It has been demonstrated that African Americans adolescents are more likely to watch television and engage in sedentary behaviors while eating, increasing risk for weight gain and by extension risk for type 2 diabetes (Kimm et al., 2001; Wolf et al., 1993). However, lack of exercise facilities, preference for social exercise regimes, and culturally based attitudes towards exercise that differ from those of White women have been cited as reasons for the lower levels of physical activity in African American women (Kumanyika et al., 1992; Lasco et al, 1989). Additionally, many women cite that if they feel that their daily work involves a significant amount of exercise, they may not be more likely to engage in further physical exercise (Melnyk and Weinstein, 1994).

Considerable research indicates that socioeconomic status (SES) at the neighborhood-level is related to obesity, physical activity, and other health-related behaviors (Gordon-Larsen, Nelson, Rage, \& Popkin, 2006). Additionally, a limited body of research has highlighted that higher accessibility to community facilities is positively associated with physical activity levels. Gordon-Larsen, Nelson, Rage, \& Popkin (2006) found that in a sample of 20,000 adolescents, major categories of physical activity-related resource were distributed inequitably. They go on to discuss that inequitable distribution is significantly associated with subsequent disparities in health-related behaviors and obesity measured at the individual level (Gordon-Larsen, Nelson, Rage, \& Popkin, 2006) In the literature, it has been acknowledged that that environmental attributes are among the least understood of known influences on physical activity (Humpel, Owen, \& Leslie, 2002). Several quantitative studies have attempted to assess the effects of environmental factors on physical activity (Humpel, Owen, \& Leslie, 2002; Mobley, Root, Finkelstein, Khavjou, Farris, \& Will, 2006). Several studies have suggested that poorer neighborhoods have been shown to be less likely to have healthy food stores, and more likely to possess fast food restaurants and higher rates of obesity (Block, Scribner, \& DeSalvo, 2004; Jeffrey, Forster, Folsom, Luepker, Jacobs, \& Blackburn, 1989; McMurray, Harrel, Deng., Bradley, Cox, \& Bangdiwala, 2000; Reidpath, Burns, Carrard, Mahoney, Townsend, 2002; Sandquise \& Johansson, 1998). Additionally, higher crime 
rates and fear for personal safety may potentially discourage women in urban environments from exercise (Ainsworth, Wilcox, Thompson et al, 2003; Eyler, MaisonKoffman, Young, et al, 2003). Ball et al. (2001) found that less aesthetic and less convenient environment was correlated with not walking; additionally, Booth et al (2000) found that footpaths safe for walking and access to local facilities was associated with being active. The CDC reported in 1999 that unsafe neighborhoods were associated with inactivity (CDC, 1999). King et al (2000) reported an interesting association between unattended dogs, hills, enjoyable scenery, and physical activity. Sallis et al. (1997) examined perceptions of neighborhood aesthetics. They found that a neighborhood environment scale, which is comprised of three separate components (neighborhood features, perceived safety, and neighborhood character), was not related to any measure of physical activity. They hypothesized that the lack of association may have been because if the neighborhood is not perceived safe, convenient, and enjoyable for physical activity, then people may be active in other environments, away from the local neighborhood. Even with these examples, the associations of environmental attributes with physical activity have thus far been evaluated in a limited number of studies, with ambiguous results (Humpel, Owen, \& Leslie, 2002). There is considerable variability in the type of intervention, duration of intervention and follow-up, the outcome measures used, and how the data are presented (Stephens, Cobiac, \& Veerman, 2014). In a review performed by Stephens, Cobiac, and Veerman (2014) where they aimed to examine the available evidence on the effectiveness of interventions targeting the treatment or prevention of overweight and obesity and those targeting improving physical activity levels across all ages, they suggest that the majority of reviews evaluating the association between obesity and physical activity presented favorable outcomes; however, not all of these outcomes reached statistical significance and many demonstrated only modest effect sizes. Dietary interventions appear to have the greatest effect on overweight or obesity, while physical activity interventions continue to be highly variable, and workplace and technology or internet-based interventions demonstrated less favorable outcomes. They also suggest that interventions that achieve a small effect in the total population, such as policy interventions, may achieve a greater overall health impact than a highly effective intervention for which only a small part of the population is eligible, such as bariatric surgery or pharmaceuticals (Stephens, Cobiac, \& Veerman, 2014). Additionally, limitations identified included publication and reporting biases, as it has been demonstrated that studies reporting positive outcomes are more likely to be published than those demonstrating negative outcomes (Stephens, Cobiac, \& Veerman, 2014).

Methodologically, one study indicated that investigators of environmental effects on health have chosen to characterize the built environment of individuals at the neighborhood scale, whether in terms of census tracts, block groups, or small buffers around individuals' homes (Ewing, Meakins, Hamidi, \& Nelson, 2014). There has been an implicit assumption within many studies evaluating the built environment that walking distance from home is the operative scale at which the built environment affects physical activity, food availability, and ultimately weight (Ewing, Meakins, Hamidi, \& Nelson, 2014). Additionally, to date, only one study has compared the power of neighborhood to predictors of obesity, and found that perceived neighborhood characteristics were more 
important than objectively measured county characteristics (Joshua, Boehmer, \& Brownson, 2008). They suggest that better measures of macro-scale characteristics such as "sprawl" are needed to represent the broad settings that shape people's health-related activities. Bauman, Sallis, \& Owen (as cited in Welk, 2002) reported derived measures that help overcome some of the methodological problems stemming from self-reported environmental measures.

Although it has been demonstrated that environments can be obesogenic by encouraging the consumption of energy dense food or discouraging physical activity or both, there still remains substantial scientific uncertainty regarding the causal relationships and the mechanisms by which environmental components operate (Poortinga, 2014). Additionally, Poortinga (2014) suggests that the literature on environmental influences on physical activity and obesity is still at an early stage of development. An analysis grid for environments linked to obesity (ANGELO) framework has been applied to examine how different economic, physical, sociocultural, and policy aspects of the neighborhood environment are linked with physical activity and obesity (Poortinga, 2014). The ANGELO framework is a grid that comprises two sizes of environment (micro and macro) on one axis and four types of environment on the other (physical, economic, political, and sociocultural) (Swinburn, Egger, \& Raza, 1999). This framework goes on to postulate that individuals interact with the environment in multiple environments, including workplaces, schools, homes, and neighborhoods.

Microenvironmental settings are influenced by broader macroenvironments, thus being less amenable to the control of individuals (Swinburn, Egger, \& Raza, 1999)

\section{Neighborhoods and their effects on health in the literature}

It has been suggested that neighborhood environment is consistently associated with obesity; changes to modifiable aspects of the neighborhood environment may curb the growth of obesity in the US and other developed nations (Michael, Nagel, Gold, \& Hillier, 2014). However, currently the majority of studies are cross-sectional and thus not appropriate for evaluating causality. One study evaluated effect of a neighborhoodchanging intervention on changes in obesity among older women using geocoded residential addresses to link data on land-use mix, public transit access, street connectivity, and access to green space from four time points between 1986 and 2004, with longitudinal data on body mass index (BMI) from a cohort of 2003 communitydwelling women aged 66 years and older (Michael, Nagel, Gold, \& Hillier, 2014). They found that there was no association between neighborhood built environment or change in built environment and BMI. Greater neighborhood socioeconomic status at baseline was independently associated with a healthier BMI at baseline, and protected against an age-related decline in BMI over time. Zhang et al. (2014) found that the link between commuting environment and obesity differed across the regional urbanization levels and suggested that urban and regional planning policies may improve current commuting environment and better support healthy behaviors and healthy community development.

Recent studies have also suggested that adults who perceive having a park, playground, or open space in their neighborhood or report that they are involved in 
neighborhood clubs, associations, or help groups have significantly decreased odds of obesity (Sullivan, Brashear, Broyles, \& Rung, 2014). Race/ethnicity also appears to affect the association between involvements in clubs, associations, or help groups and obesity. Additionally, when stratified by race/ethnicity, report of involvement in clubs, associations, or help groups is significantly associated with a decreased odds of obesity among Non-Hispanic white adults, further showing differences in this association by race/ethnicity. The perceived presence of a park, playground, or open space was only significantly associated with decreased odds of obesity among African Americans in the stratified analysis by race/ethnicity. However, the interaction term of race/ethnicity and perceived presence of a park, playground, or open space was not statistically significant, showing no differences in this association by race/ethnicity. These results imply that providing or increasing perception of parks, playgrounds, or open space may decrease the odds of obesity, especially in ethnically diverse neighborhoods which can be an area of concentration for policies and obesity prevention (Sullivan, Brashear, Broyles, \& Rung, 2014). They also suggest that disparities in neighborhood environments through selfreported (perceived) measures may contribute to increased prevalence of obesity among African Americans. Findings from this study suggest that providing or increasing the perception of parks, playgrounds, or open space may decrease the odds of obesity, especially in ethnically diverse neighborhoods. Additionally, the presence of higher household density, closeness to rail stations, and better bus services have been found to be associated with a lesser likelihood to be obese, which implies that people in a welldesigned transit-oriented neighborhood tend to use active transportation modes to reach their daily activities and access transit services. Increased use of active transportation gives people an opportunity to engage in a moderate-level of exercise, so as to reduce their weight and enhance their health condition (Hu, Cho, Huang, Wen, Choi, Shih, \& Lightstone, 2014)

Conversely, the study conducted by Powell-Wiley and associates indicated that the presence of drug problems in the neighborhood increased the odds of obesity, although the results were not statistically significant, and the presence of crime did not increase the odds of obesity. These results are similar to another study that did not find a significant association between obesity and a perceived measure of neighborhood violence (Powell-Wiley, Brashear, Broyles, \& Rung, 2014). Perceived safety from crime was associated with lower BMI in another study on the influence of the social environment among an Australian adult population (Christian, Giles-Corti, Knuiman, \& Timperio, 2011). These inconsistent findings were speculated to be due to differences in methodologies, construction of composite scores of crime, or differences in constructs regarding crime definitions (Powell-Wiley, Brashear, Broyles, \& Rung, 2014).

Another study conducted in Canada examined the complex relationships between individual demographics and behaviors, and aspects of the local social and physical environments, using a subset of a nationally representative survey linked to neighborhood-level data from the 1991 Canadian Census (Harrington \& Elliott, 2009). This study found substantial area-level variation in body mass index and waist circumference, and discovered an important role for neighborhood-level characteristics independent of individual-level characteristics. The authors suggest that these findings 
provide evidence that the underlying mechanisms driving the increasing prevalence of overweight and obesity may be obesogenic environments that encourage physical inactivity and unhealthy eating (Harrington \& Elliott, 2009).

Supermarkets have been identified as components of the physical environment that may influence food choices, and more distal outcomes like obesity, by increasing the availability of healthy foods. Availability and accessibility to food sources may play a significant role in dietary quality and food choices and so has an important role in regulating risk for disease. Although in the literature, supermarkets are found to offer a greater selection of fresh healthy food items and heart healthy foods (Galvez, et al., 2007) recent evidence regarding their effects is ambiguous, because supermarkets also increase the availability of unhealthy options (Hutchinson, Bodor, Swalm, Rice, \& Rose, 2012). Hutchinson et al. (2012) developed an alternative measure of food environment quality that characterizes urban neighborhoods by the relative amounts of healthy (e.g. fruits and vegetables) to unhealthy foods (e.g. energy-dense snacks) using data from 307 food stores and 1243 telephone interviews with residents in urban southeastern Louisiana. They found that higher quality food environments decreased the risk of obesity and overweight, generating statistically significant results (Hutchinson, Bodor, Swalm, Rice, $\&$ Rose, 2012). While supermarkets have been found to have two times the amount of heart-healthy food than neighborhood grocery stores and four times the number of these foods in convenience stores (Morland et al, 2002), convenience stores have been shown to have very few low-sodium, low fat foods (Sallis, et al., 1986). Low income and predominately African American neighborhoods have been shown to have poor access to healthy food resources (Zenk et al, 2005). In a study of the prevalence of supermarkets by neighborhoods, Morland et al found that four times fewer supermarkets were located in African American neighborhood than in White neighborhoods (Morland, 2002). Similarly, African American neighborhoods were seen to have half as many chain supermarkets as compared to White neighborhoods (Powell, 2007). Reports show that black Americans consume one third more fruits and vegetables for every additional supermarket found in their census tract (Morland et al., 2000).

It was found that, $30 \%$ of the food stores in high poverty, predominantly African American neighborhoods were seen to lack sufficient amounts of fruits and vegetables compared to predominantly White neighborhoods (Sloane, 2006). In a comparable study, nonwhite Albany residents lived in neighborhoods that lacked stores that contained lowfat milk or high-fiber bread (Hosler, 2006). One study found that the nearest supermarket was significantly farther away in predominantly African American neighborhoods compared to a predominantly White neighborhood (Zenk, 2005).

However, unlike supermarkets, fast food restaurants are found frequently in African American neighborhoods. In a study in New Orleans, researchers found that in predominantly African American neighborhoods, there were 2.4 fast food restaurants per square mile compared to 1.5 fast-food restaurants found in White neighborhoods (Block et al., 2004). It has been shown that full-service and fast-food restaurants were $58.2 \%$ and $59.3 \%$, more available in predominantly African American neighborhoods respectively, compared to predominantly White neighborhoods (Powell et al, 2007). Lewis et al found 
that fast-food restaurants in African American communities heavily promoted unhealthy food options to attract residents to eat at their restaurant, and were significantly less likely to promote healthy items (Lewis et al., 2005). Fast food restaurants are more common in African American communities, further suggesting an unhealthy African American food environment.

Socioeconomic status plays a significant role in healthy food stores' accessibility and availability. Distributions of food stores are thought to follow the distribution of race and wealth, creating neighborhood environments with concentration of poor African Americans with insufficient number of supermarkets and high numbers of fast food restaurants (Zenk et al, 2005). Some studies suggest that migration of supermarkets to the suburbs and the lack of transportation available to low-income communities contributes also to the health disparity found in minority communities ("Urban grocery gap", 1992) ("Food Security in the United States", 1987). The decrease in supermarkets are said also force residents to depend on smaller stores such as grocery and convenience stores which have less healthy food choices (Curtis and McClellan, 1995). While there are social interpersonal factors that may apply to the African American food experience such as food preferences, eating habits and knowledge of food nutritional values, without access to healthy food sources, the ability of African Americans to participate in healthy eating behaviors decreases significantly because their lack of access to healthy food sources (Zenk, et al, 2005).

The consistent trend within the body of research evaluating the effects of neighborhood on environment suggest that multiple factors can effectively contribute to, or discourage physical activity and increase options to produce healthy behaviors are correlated with improvements in BMI and health. The walkability of neighborhoods and availability of and proximity to neighborhood amenities, parks, and recreational facilities can affect opportunities for discretionary and incidental physical activity. The proximity to supermarkets and availability of fruits and vegetables affects accessibility to healthy food options. Additionally, social characteristics of the environment such as neighborhood safety, crime, and drug problems can also influence obesity through determinants of physical activity behavior. Inequalities in the built environment and neighborhood social characteristics may contribute to racial/ethnic disparities in the prevalence of obesity and type 2 diabetes (Sullivan, Brashear, Broyles, \& Rung, 2014).

\section{Part 3: The Socio-Ecological Model, a Review of the Literature, Conceptual Framework, and Relevant Applications}

The socioecologic conceptual model describes a chain of influences that may impact health outcomes. The hierarchy includes macro-level fundamental factors such as racial/ethnic segregation and education disparities that affect intermediate factors such as land use, transportation systems, and the availability of parks, shopping, recreational, or healthcare facilities (Mobley, Root, Finkelstein, Khavjou, Faris, \& Will, 2006). These in turn may impact more proximate factors, such as environmental stressors (e.g.: crime, safety), health behaviors (e.g.: dietary practices, physical activity), and social integration 
or support systems. The last level in the hierarchy is at the individual level, where health outcomes such as obesity and cardiac disease are ultimately affected by all factors from the higher levels of the socioecologic hierarchy.

One study utilizing the socioecological conceptual framework examined whether certain characteristics of the built environment were associated with obesity or coronary heart disease (CHD), and found that socioecologic characteristics of financially disadvantaged women were associated with BMI and CHD risk (Schutchfield \& Howard, 2011). Another study evaluated the significance of socioecologic and psychosocial characteristics as predictors of incidents of racial discrimination. The researchers of this study found that as education increased, the likelihood of reporting racial discrimination increased; additionally, urban respondents were more likely to report racial discrimination compared to suburban respondents (Brown \& Davis, 2003). Additional research utilizing the socioecological model has attempted to identify factors perceived as influencing overweight and obesity, finding that cultural norms of overweight and obesity and cultural food preferences among Native American and African American women were identified as perceived contributors (Layton, Parker, Hermann, \& Williams, 2009). The researchers also suggested that identification of cultural differences and perceived needs from the perspective of limited income women may benefit the development of future programs. Furthermore, focusing on environmental factors, as opposed to individual factors, may be more influential in the development of programs to address overweight and obesity (Layton, Parker, Hermann, \& Williams, 2009).

The social ecological perspective suggests that interventions at multiple levels of the model are needed to address obesity, particularly among vulnerable groups. As described by Washington State Partners in Health (n/a), this section gives an overview of the socioecological model and describes factors that researchers have linked to obesity at each level of the model.

\section{Individual level}

- Physiology: genetic predispositions to obesity and other physiological factors that may contribute to obesity, including satiety and resting metabolic rate.

- Individual activity: an individual's level of recreational, occupational and transport activity, and activity patterns.

- Individual psychology: self-esteem, personal stress, and change predisposition

\section{Organizations and public policy level}

- Environment for physical activity: cost of physical activity, perceived safety and walkability. 
- Food consumption: characteristics of the food environment including level of food abundance and variety, quality food items, food energy density, and portion size.

- Food production: environmental food demand, which drives growth and profitability, food market price, and productivity efficiency

\section{Community level}

- Social psychology factors: education, and societal attitudes

Several principles are incorporated in the framework of the socioecological model, including the ability to peform multidimensional analyses, examine differential dynamic interplay, insight into systems theory, recognition of the interdependence of environmental conditions, and the model's inherent interdisciplinarity. As described by Binder, Stokols, and Catalano (1975), the principles of the socioecological framework are listed below:

\section{Principle one: Multiple dimensional analysis}

Environmental settings have multiple dimensions that may influence and affect personal environment interaction.

Principle two: Differential dynamic interplay

Environmental factors may affect people differently depending on factors such as personality, health practices, personal perceptions of control over environmental factors, and personal economic resources.

Principle three: Relevance of systems theory

The whole system may be greater than the sum of its parts, and interventions uniting the whole may be more effective than targeted individual interventions.

Principle four: Interdependence of environmental conditions

Multiple settings affect an individual's well-being and it is important to consider the links between the social and physical aspects of the environment.

Principle five: Inherent interdisciplinarity

Social ecology analyses emphasize the integration of multiple levels of analysis with diverse methodologies.

Stokols (1996) examines three theoretical perspectives on health-promotion and indicates that the environment is assumed to function not only as a potential source of 
pathogens, toxins, and safety hazards, but also as a provider of health-promotive information and social support that can enable people to achieve higher levels of wellbeing than implied by the term "health-promotion". The author also suggests that the theoretical underpinnings of social ecology are relevant to several practical issues that arise in attempts to design effective community interventions (Stokols, 1996). However, research has also indicated that principles of the socioecological model are difficult to translate efficiently into action (Stokols, 1996). Based on ecological principles, interventions require the combination of interdisciplinary knowledge, requiring substantial coordination among community members and stakeholders (Stokols, 1996). Stokols (1996) also suggests that combining active and passive interventions for health promotion and incorporating multi-level, multi-method assessments of program outcomes can be expensive and logistically complex; this raises important questions about the potential over-inclusiveness and utility of ecologically oriented health promotion programs. Practically, if ecological models are interpreted as completely encompassing conceivable health variables relating to the environment, their utility for intervention and research basis may be substantially reduced (Stokols, 1996). In other words, targeting specific interventions based on the health state of interest, the determinants found among those specific individuals, and the specifics of that particular environment may be a more effective strategy that generates significant results.

This study addresses many aspects found within the socioecological framework. A pertinent and perhaps distinguishing quality of this work incorporates a quantification of the walkability of the study area, along with perceptions regarding social psychology, individual psychological factors, food availability, and individual physiological characteristics. Walkability is a measure of the degree to which the built environment is friendly to the presence of people living, shopping, visiting, enjoying, or spending time in the area (Abley, 2005). Features of the environment affecting walkability include, but are not limited to land-use mix; how connected neighborhood streets are, residential density (residential units per area of residential use), and places to go near identified residential communities (Daniel \& Perrotta, 2009). Infrastructural factors that potentially affect walkability include access to mass transit, availability and quality of sidewalks, buffers to moving traffic, pedestrian crossings, aesthetic characteristics, and proximal local destinations, air quality, the presence of shade in appropriate seasons, and traffic (both speed and volume) (Abley, 2005). It also includes diversity of people, especially the presence of children, seniors, and people with disabilities, and denotes the quality of a walkable space (Abley, 2005). These factors have been shown to influence whether or not residents' proximal neighborhoods are used for physical activity. Rattan, Campese, and Eden (2012) automated an analysis procedure where walkability was quantified. Their methods depicted how three factors (density, diversity, and design) can provide valuable information regarding an area's walkability. They also found that assessing these walkability maps provided a context for determining whether areas required better design to increase access and walkability, or marketing to promote walking in highly walkable areas (Rattan, Campese, \& Eden, 2012).

However, it is important to mention that neighborhood walkability is only one aspect of a very complex issue. Neighborhood effects, or concentration effects, can occur 
as poverty is increasingly concentrated in certain neighborhoods (Teitz \& Chapple, 1998). As the incomes of neighborhood residents decline to levels unable to support a viable retail sector or to maintain housing, a spiral of abandonment and decay can occur (Teitz \& Chapple, 1998). When the area becomes a high-poverty neighborhood, average educational attainment levels decrease, and teenage pregnancy and high school dropout rates may increase dramatically (Teitz \& Chapple, 1998). Additionally, segregation itself can cause poverty and propagation of misinformation, also exacerbating negative health status. 


\section{CHAPTER 3. METHODS}

\section{Study Design}

This research study was guided by Lincoln and Guba's naturalistic inquiry methodology (Lincoln \& Guba, 1985) using an interpretive approach to describe, analyze, and understand weight loss and health maintenance in the context of environmental factors amongst low-income African American women with type 2 diabetes. To increase the strength and rigor of this qualitative investigation, three types of triangulation were employed, outlined in Figure 3-1: methodological (semi-structured interviews and document analysis), Geographic Information Systems, and field notes from direct environmental observation to inform the assessment of resource quality. Geographic Information Systems was used to quantify walkability, proximity of resources in the ZIP codes 38109 and 38106, and to assess land-use patterns and availability of institutional resources. Concurrent with this data collection, qualitative individual interviews using a form of naturalistic inquiry explored participant perceptions of their health, their weight loss experiences, their perceptions of the proximal environment, and how their perceptions may affect environmental utilization for physical activity.

\section{Study Sample Composition}

The study sample consisted of 20 women (11 from 38106; 9 from 38109) who were recruited to participate in individual interviews to reach thematic saturation. The 3549 age group was selected because at this age, women have a more difficult time losing weight; before this age a different set of circumstances and weight loss intentions may apply; after this age, generally hormonal and health changes also present a different set of circumstances for women.

In the qualitative portion of the study, purposeful/selective sampling was used, where a specific set of criteria was used to identify and recruit participants of interest. Eligibility questionnaires were conducted over the phone; in-depth interviews were conducted in person; member checks were conducted over the phone. Three women from 38106 and three women from 38109 initially interviewed were contacted to conduct follow-up member checks. In-depth interviews were approximately 20 - 45 minutes, with 3 interviews lasting approximately one hour. Participants were composed of people identified as residing in the ZIP codes 38109 and 38106, recruited from the Endocrinology, Diabetes, and Metabolism clinic at The Medplex Outpatient Center. This recruitment site was chosen because The Medplex Outpatient Center typically services vulnerable populations that are low-income, as well as its general proximity to the ZIP codes of interest. Recruitment consisted of flyers placed at clinic sites; database recruitment; screening questions; the researcher completing an eligibility questionnaire for each participant to indicate eligibility; and participant consent of study participation. 


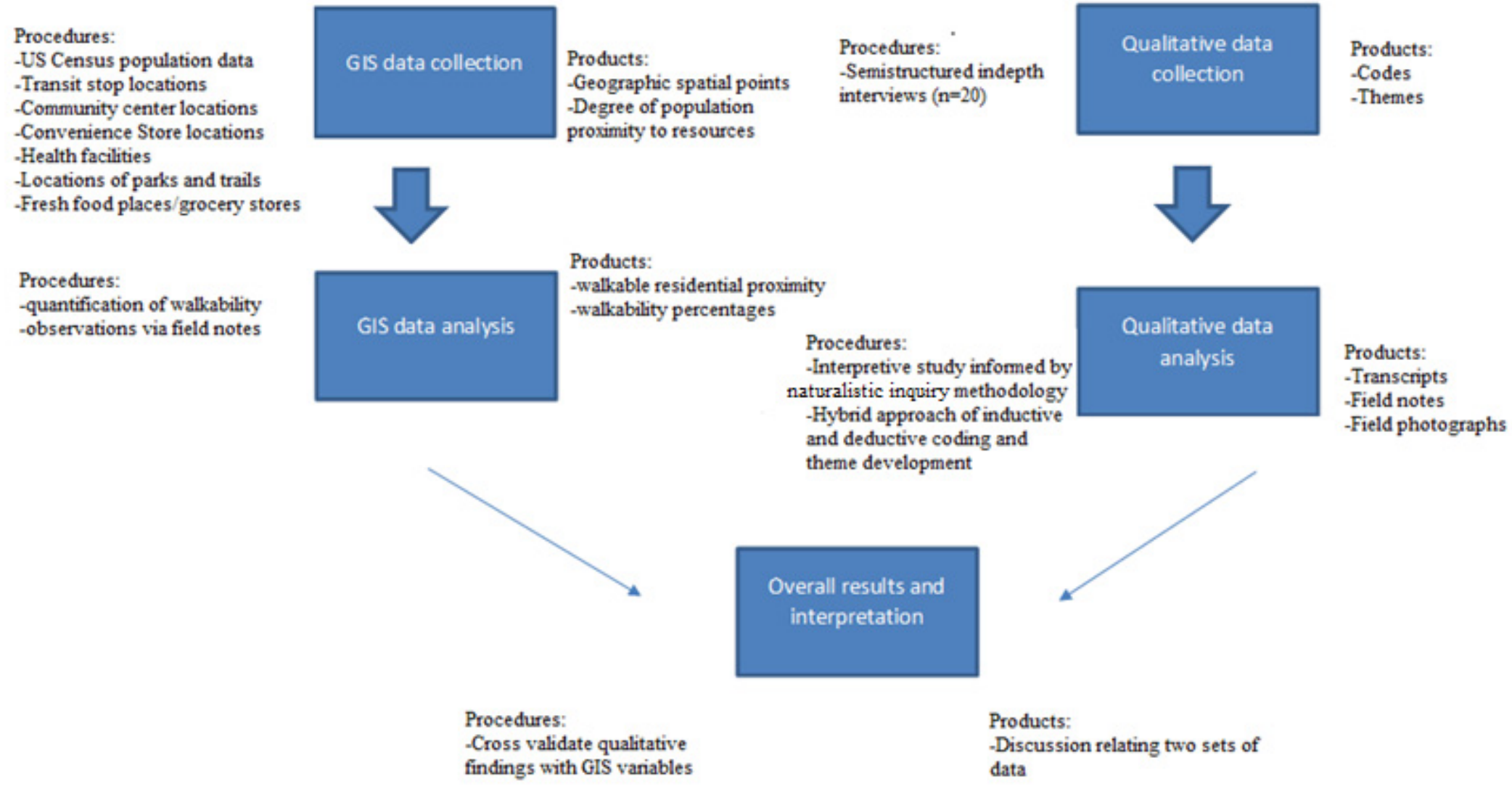

Figure 3-1. Interview and GIS data triangulation. 
Patient lists were obtained from The Medplex Outpatient Center subsequent to IRB consent. Phone numbers were acquired through database search, after database access requests to in-hospital systems were approved by The Medplex Outpatient Center. From the patient criteria listed below, patient lists consisting of 130 potential participants from 38109 and 107 potential participants from 38106 were generated.

Inclusion criteria of purposeful sample:

1. Women, whose income level was determined via questionnaire and is $125-200 \%$ of the federal poverty level (FPL);

2. African American;

3. 35 to 49 years of age;

4. Identified residents or previous residents of ZIP codes 38109 and 38106, with confirmation via eligibility questionnaire;

5. A diagnosis of type 2 diabetes. IRB consent was obtained prior to recruitment methods and was verbally received from each participant who agreed to participate; and

6. Any visit or inpatient admission into The Medplex Outpatient Center, occurring anytime over a 5 year period, beginning in 2008 .

\section{Data Collection and Instrumentation}

Women were interviewed under conditions that were as authentic as possible. Most often, women were interviewed face-to-face in their households to not only aid in their comfort, but to observe their home and proximal daily environments. Women were interviewed during August 2013 and January/April 2014. Interview/observation sessions ranged from thirty to sixty minutes and during that time, qualitative data about participants' experiences were gathered through interview questions. All interviews were tape-recorded and conducted by the same interviewer using a semi-structured questionnaire. The determination of eligibility, verbal informed consent, and interview procedures are detailed in Figure 3-2.

The semi-structured questionnaire consisted of 9 items, inquiring about participants' experiences of diabetes, weight loss importance (including goals set, successes or failures, difficulties in health maintenance), length of time in neighborhood and perception of neighborhood, perception of active neighbors, and utilization of neighborhood for physical activity (walking, running, exercise with neighbors).

The development of the survey instrument was evaluated and validated by the student's dissertation committee. Additionally, after the initial interviews were 


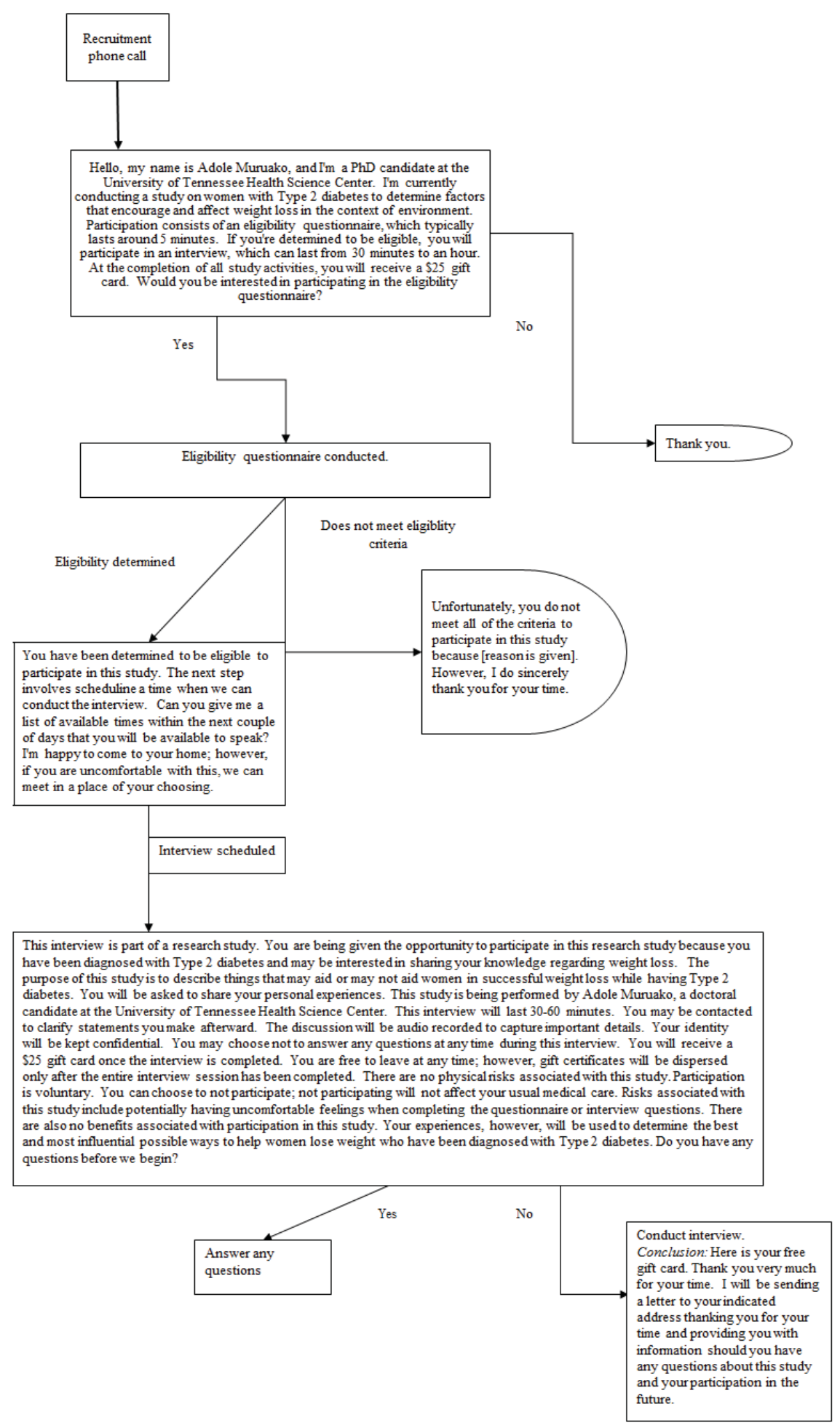

Figure 3-2. Eligibility determination, informed consent, and interviewing procedures. 
conducted, the scope of data collection was refined using constant comparative analysis. Categories arising from this method are noted to take on two forms - those that are derived from the participants' customs and language, and those that the researcher identifies as significant to the project's focus-of-inquiry; the goal of the former "is to reconstruct the categories used by subjects to conceptualize their own experiences and world view", the goal of the latter is to assist the researcher in developing theoretical insights into the social processes pertinent to the phenomenon. Lincoln and Guba (1985) further suggest that "the process of constant comparison stimulates thought that leads to both descriptive and explanatory categories" (pp 334-341). Categories undergo content and definition changes as units and incidents are compared and categorized, and as understandings of the properties of categories and the relationships between categories are developed and refined over the course of the analytical process. Three distinct versions of the interview guide were developed as a result of the constant comparison technique.

Three women from 38106 and three women from 38109 were contacted in April/May 2014 for member checks. Each member check was conducted over the phone. The selected women were informed of the data analysis results and were asked to verify whether the information accurately reflected their experiences. Each woman did verify that in their opinion, the analysis results were accurate in depicting the experiences shared among the study population.

\section{Data Analysis}

\section{Generating codes and behavior domains from interview responses, phase 1 coding guide}

The ultimate goal of the interviews was to identify emerging themes in order to analyze and describe the experiences of African American women who possess type 2 diabetes. More specifically explored were perceptions of their health; their perceptions of their environment; whether their perception of the environment affects their utilization of the environment; and whether objective environmental measures are reflected through their perceptions. Further explored was the presence of substantial environmental barriers to weight loss, physical activity, and health maintenance.

To begin the coding, transcripts were reviewed, and salient, essence-capturing elements of interview responses were developed comprising each code. Codes serve as the "bones" of analysis, ultimately assisting in the reduction and integration of conceptual information into patterns held within responses. One of the goals of the coding process was to capture patterns of similarity among respondent population, as well as patterns of difference with respect to environmental perceptions between the 2 groups. The following behavior domains were derived from the research questions, the initial and secondary coding process of interview responses, and comprised the categorization of the data during thematic development: 
1. Economic Factors:

i. Encouraging physical activity, weight loss, health maintenance

ii. Discouraging physical activity, weight loss, health maintenance

2. Environmental Factors:

i. Encouraging physical activity, weight loss, health maintenance

ii. Discouraging physical activity, weight loss, health maintenance

3. Sociocultural Factors:

i. Encouraging physical activity, weight loss, health maintenance

ii. Discouraging physical activity, weight loss, health maintenance

4. Methods of weight loss

5. Mental considerations

6. Education/knowledge considerations

The coding process was cyclical in nature, reflecting the emergent design of naturalistic inquiry; as interviews were conducted, coding and recoding responses also refined the interview questions.

Once a response that related to a domain was identified, that particular response was marked using the code created for that domain. Discussion amongst the researcher and the experienced committee member regarding thought process during coding procedures has been indicated in qualitative methodology as vital to the analysis process. After the entire document was coded during Phase I of coding, codes were reviewed to ensure that the researcher and committee member working on the analysis were in agreement with the coding results. Once no new codes or behavior domains were identified, Phase 2 of the coding guide commenced. The process was as follows:

1. Each interview response was evaluated and an essence-capturing phrase was assigned to pertinent concepts.

2. Responses were recoded as participants were recruited and interviewed

3. Behavior domains were developed.

4. Responses were cyclically categorized according to behavior domains

5. Codes and concepts generated during interviewing were discussed.

6. Recoding produced no new codes or concepts, initiation of Phase 2 of the coding guide occurred. 


\section{Categorizing codes into themes using behavior domains, phase 2 coding guide}

During thematic development, behavior domains were created on posters, and each generated code was cut and pasted from the transcript onto the identified corresponding behavior domain, taking care to continuously refer to the research questions as the data was evaluated. Ultimately, this process was to assess visually how each code linked to each domain as the analysis procedure proceeded.

Using the Phase I portion of the coding guide, responses to each question were read through, overarching codes were assigned to ideas and perceptions, and through this process emerging categories and patterns as they related to the perception of health and connections of these perceptions with weight loss, health maintenance, utilization of the environment for physical activity were identified. Follow-up interviews and member checks were performed to ensure conceptual accuracy, to assess trustworthiness of accounts, and to check interpretations. Analytic memos during member checks and analysis served to maintain reflexivity throughout the collection and analysis process. Response frequency identified major and minor inductive categories generated in the analysis process. This process is listed below:

1. Using the Phase I portion of the coding guide, the responses to each question were evaluated, looking for emerging categories or patterns as they related to the behavior/content domains.

2. Member checks were performed to ensure and validate interpretation accuracy.

3. Analytic memos were developed to encourage reflexivity.

4. Major and minor inductive categories were generated during the analysis process using response frequency.

\section{Data Interpretation}

Following the development of categories within each identified behavioral domain, explicit links between the data and the interview objectives were made, using patterns among participants' responses. More specifically, themes were developed from major inductive categories. Throughout the analysis process, hypotheses were generated based on data observation, where a search of all responses was undertaken to locate any negative or contradictory cases. In addition, identification of any distinctive or unusual views, ideas or opinions in relation to the factors that influence behavior among study participants was noted. The development of inductive categories allowed grounding of these categories to the data from which they derived, thematic development, and ultimately, theory.

The following questions guided data interpretation: 
1. "What was known and then confirmed or contested" by the interview data?

2. "What was suspected and then confirmed or contested" by the interview data? Were any of the suspicions regarding potential factors influencing the behavior of the target population identified during the interviews? Were any of the suspicions refuted?

3. "What was new that wasn't previously suspected?"

Following the flow of naturalistic inquiry, qualitative results were reported in a thematic, case-study format and presented in Chapter 4.

\section{Geographic Information Systems Methods}

The purpose of the Geographic Information System (GIS) methods was to triangulate participant responses with environmental data, staying true to the process of naturalistic inquiry. A secondary purpose of this analysis was to highlight the impact of community design on levels of physical activity and social cohesion, and identify areas of potential improvement. The GIS methods were adapted directly from the methods of Rattan, Campese, and Eden (2012) in which walkability was quantified. As indicated in these methods, data collected to build the models included the 38109 and 38106 ZIP code tabulation areas as polygons; streets corresponding to these areas were obtained as lines; residential locations with population information were gathered as points and polygons; and area resources of interest were collected to be depicted as points.

ZIP code tabulation areas were collected from the US Census (US Census Bureau); street lines were collected from the US Census as TIGER/Line shapefiles; population information by block was obtained from US Census information via American Factfinder; transit stop location information was obtained from the Memphis Area Transit Authority; resources of interest in these areas included grocery stores, fresh food places (farmers markets), convenience stores, parks and trails, community centers, health facilities, and transit stops; most of these were gathered through internet searches, telephone book verification, and resident accounts. A geodatabase containing addresses of grocery stores, fresh food places, convenience stores, schools, parks and trails was built using Microsoft Excel and imported into ArcCatalog. After using the Shelby County streets layer to create an address locator, the ArcGIS geocoding tool converted all resource addresses into spatial points.

The next steps involved determining the percentage of residents in 38109 and 38106 who live a walkable distance from resources of interest. Each resource was input as a parameter to determine walkable distance, depending on the resource of focus. The workflow steps included:

1. Calculating and assigning the total residential population for 38109 and 38106 ; 
2. Calculating the service area for each resource of interest using the Network Analyst tool;

3. Selecting blocks that intersect the service area for each resource of interest;

4. Calculating the percentage of residents in 38109 and 38106 with walkable access to each resource of interest;

5. Classifying and visualizing walkability for each ZIP code by block.

In calculating the total residential population, US census files obtained by American Factfinder were generated to depict population information by block. This information was linked to the block shapefile, also obtained by the US Census according to each block's unique geographic identifier. Census tract information was required to generate the link between the ZCTA shapefile and the block shapefile. Block polygons were converted to block points using the Feature to Point tool to aid in spatially joining the ZCTA and blocks. Modelbuilder was used to calculate the total sum of the residents that reside within the ZCTA, generated by adding together all blocks present within the ZCTA. This model is depicted in Figure 3-3. The resulting shapefile included the total population of the ZCTA within the attribute table and was labeled ZCTA_pop.

In calculating the service area for resources of interest, walking distance criteria was based on criteria established in the methods of Rattan, Campese, and Eden (2012) and is depicted in Table 3-1. More specifically, 400 meters approximately represents a 5 minute walk; 800 meter represents a 10 minute walk, etc. ModelBuilder within ArcGIS 10.1 was used to design an algorithm that calculated the service area for each resource, using the Shelby County street network. In ArcCatalog, the Shelby County street layer was used to create a new network dataset for network analysis. After the service area was calculated it was exported as a polygon layer. The model used to calculate the service area polygons is represented in Figure 3-4. The 'Select Facility' parameter was used to specify the resource for which each service area was to be calculated. Created service area polygons were merged and trimmed to 30 meters. According to the aforementioned reference, this distance is determined to be the best for ensuring that only residential parcels identified as walkable to the resource were selected. To select for residential parcels that intersect the service area for each resource, selected parcels are converted to residential parcel points to prevent walkable parcels that fall on ZCTA borders. This prevented borders from being included in multiple ZCTAs. The percentage of residents in each ZCTA with walkable access to resources of interest are calculated by dividing the proportion of residents within the service area by the total population within the ZCTA. This was accomplished within the Calculate Field tool. Additional GIS methods included demonstrating ZIP code connectivity by creating lines depicting sidewalk access. 


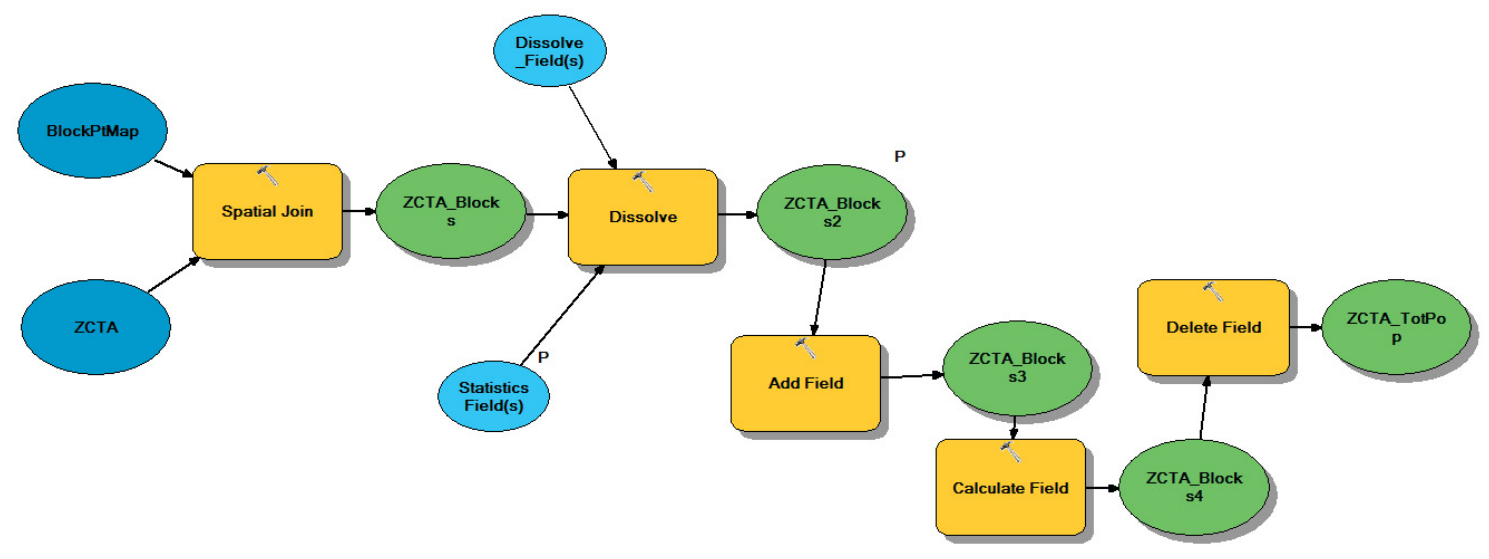

Figure 3-3. Model used to generate total ZIP code population.

Table 3-1. Resources of interest and associated walking distance criteria.

\begin{tabular}{ll}
\hline Resource of interest & $\begin{array}{c}\text { Walking } \\
\text { distance criteria }\end{array}$ \\
\hline Transit stops & $\leq 400$ meters \\
Grocery stores & $\leq 800$ meters \\
Fresh food places & $\leq 800$ meters \\
Convenience stores & $\leq 400$ meters \\
Parks/Trails & $\leq 800$ meters \\
Health care facilities & $\leq 1200$ meters \\
Community centers & $\leq 800$ meters \\
\hline
\end{tabular}




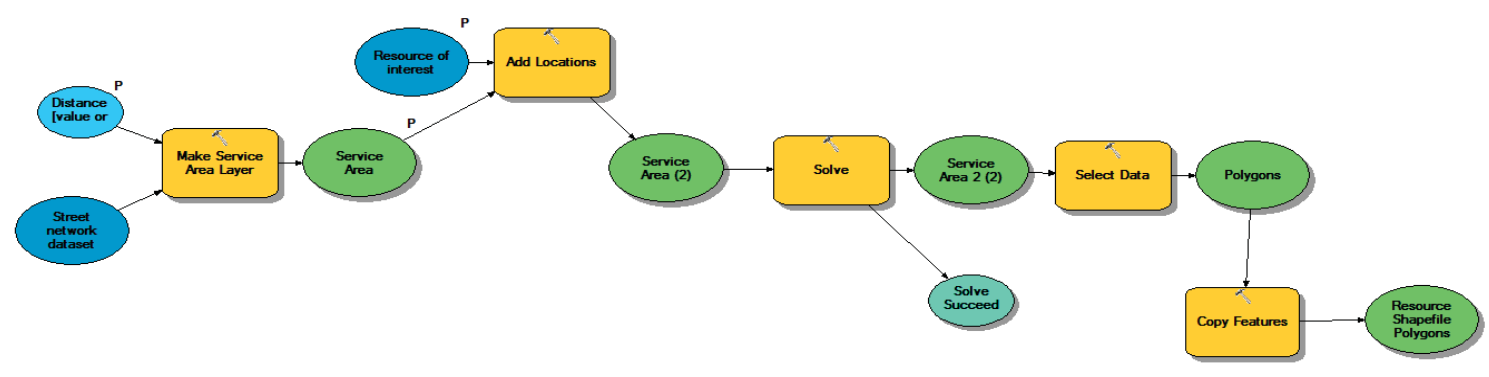

Figure 3-4. Model used to generate service area polygons. 


\section{CHAPTER 4. RESULTS}

The findings of this study, which examined cultural, social, economic, and environmental influences that encourage or discourage successful weight loss and physical activity, action strategies used by the respondents to achieve potential weight loss, and objective components of the physical environment using Geographic Information Systems are presented in this chapter. The findings also consist of the respondent's demographic information and the data analysis, presented in four parts.

The units of analyses for this study consist of the research questions presented in Chapter 1:

1. What are methods of weight loss, including forms of environmental utilization, employed by low-income, African American women with type 2 diabetes mellitus in the 38106 and 38109 ZIP codes of Memphis, TN?

2. What are the sociocultural, economic, and environmental influences that encourage or discourage successful weight loss, health maintenance, and physical activity among low-income, African American women with type 2 diabetes mellitus in the 38106 and 38109 ZIP codes of Memphis, TN?

3. What are objective environmental barriers to achieving weight loss that can be gauged through Geographic Information Systems?

Analysis of the interview data as presented in this chapter was achieved by the use of a coding guide, which served as a data management tool for organizing portions of related text and to assist in interpretation. The use of a guide provided a clear trail of evidence for the credibility of the study. Additionally, triangulation of the interviews, GIS maps, field notes and photographs sought to answer the research questions. The semi-structured interview data analysis answered questions one and two. GIS maps, field notes, and photographs answered question three.

Relevant block quotations to substantiate thematic identification will be used, following the guidelines provided by the $6^{\text {th }}$ edition of the American Psychological Association Citation Style Manual. To protect the privacy of respondents, a numeric code, labeled as the Participant ID, was assigned to each participant, and will be used to identify the source of quotations.

\section{Demographic Information}

Of 237 women determined to fit the inclusion criteria of the purposeful sample, each potential participant was contacted via phone to introduce to and inform of the project, to inquire about interest in study participation and to potentially conduct initial eligibility questionnaires. Of potential participants in both 38109 and 38106, 59 women 
did not answer either on the primary call or on callback, 66 contact numbers were disconnected, there were 6 wrong numbers, and 49 women declined to participate in the study. Eligibility questionnaires were conducted on 37 potential participants. Of these, 6 were ineligible because their ZIP code had been misrepresented in the database file, and 1 was deemed ineligible because her daughter indicated that she had recently passed away.

Ten women who were eligible to participate and who agreed to a scheduled time either later declined to participate, had unforeseen scheduling conflicts, or there was a nonresponse to follow up which resulted in an incomplete. A total of 20 respondents consisting of 11 women from 38106 and 9 women from 38109 agreed to participate in face-to-face semi-structured interviews, including an eligibility questionnaire conducted via phone, and comprise the final study sample. Tables 4-1, 4-2, 4-3, and 4-4 summarize the demographic information of the 20 participants of the study.

The average participant age in the study sample was 43 years. Average length of time that each participant lived in their corresponding residential neighborhoods was 10.25 years. The most commonly indicated comorbidity was hypertension, with 12 women noting this condition. Amount of weight loss/gain since diagnosis was gauged via eligibility questionnaire. Most women (16) indicated that they had lost weight since being diagnosed with diabetes, with the average reported weight loss among participants to be approximately 21 pounds. Only 4 participants indicated weight gain. Although many participants indicated that they had lost weight since diagnosis, several of the participants remained overweight/obese despite reporting weight loss.

Comparing the two study areas, the average age of study participants in 38109 was slightly higher at 45 years compared to 42 years among 38106 participants. Additionally, the average reported BMI among 38109 participants was slightly higher. Statistically, participants from 38106 were residents of the ZIP code for a longer period of time at an average of 12 years, when compared to participants from the 38109 ZIP code, who reported an average of 6 years residence. Residents from 38106 also reported substantially greater amounts of weight loss since diagnosis, at approximately 30 pounds, when compared to women from 38109, who lost an average of approximately 10 pounds since diagnosis.

Figure 4-1 presents the constant comparison workflow of the data collection process. During constant comparison, the interview guide was initially refined, reflecting version 2, after 4 women were interviewed to best reflect the quality of responses generated from participants, and to obtain the most relevant and information-rich responses. Additionally, potential emergent thematic elements were initially identified from the first round of interview responses to determine whether any major themes were present. A third version of the interview guide was developed, along with emergent thematic and inductive analysis, after additional interviews from women from both the 38106 and 38109 ZIP codes were obtained. 
Table 4-1. Demographic characteristics and related variables of study respondents.

\begin{tabular}{|c|c|c|c|c|c|c|c|c|}
\hline $\begin{array}{l}\text { Participant } \\
\text { ID }\end{array}$ & Age & $\begin{array}{c}\text { Indicated } \\
\text { weight, lbs. } \\
\text { (height) }\end{array}$ & $\begin{array}{c}\text { Year of } \\
\text { diagnosis }\end{array}$ & $\begin{array}{l}\text { Body } \\
\text { Mass } \\
\text { Index }\end{array}$ & $\begin{array}{c}\text { ZIP } \\
\text { Code }\end{array}$ & $\begin{array}{l}\text { Length of } \\
\text { time in ZIP }\end{array}$ & $\begin{array}{c}\text { Cumulative } \\
\text { weight } \\
\text { loss/gain (lbs.) } \\
\text { since diagnosis }\end{array}$ & $\begin{array}{l}\text { Comorbidities } \\
\text { indicated }\end{array}$ \\
\hline 001 & 40 & 159 (5'1”) & 2008 & 30 & 38106 & 2 years & +60 & Bipolar disorder \\
\hline 002 & 48 & 162 (5'3’') & 2001 & 28.7 & 38106 & 48 years & -90 & Pancreatitis, thyroid \\
\hline 003 & 47 & 215 (5'6’) & 2006 & 34.7 & 38106 & 17 years & -10 & $\begin{array}{l}\text { Hypertension, sleep } \\
\text { apnea }\end{array}$ \\
\hline 004 & 41 & 239 (5'4’) & 2004 & 41 & 38106 & 22 years & -65 & $\begin{array}{l}\text { Thyroid, Graves, } \\
\text { hypertension }\end{array}$ \\
\hline 005 & 39 & $180\left(5^{\prime} 6^{\prime \prime}\right)$ & 2006 & 29 & 38106 & 7 years & -30 & $\begin{array}{l}\text { Hypertension, high } \\
\text { cholesterol }\end{array}$ \\
\hline 006 & 40 & 342 (5'4’') & 2001 & 58.7 & 38106 & 4 years & -30 & None \\
\hline 007 & 38 & 158 (5'4’') & 2010 & 27.1 & 38106 & 3 years & -7 & Oral cancer \\
\hline 008 & 46 & 165 (5'7'’) & 2010 & 25.8 & 38106 & 1 year & -50 & High cholesterol, \\
\hline 009 & 41 & 293 (5'4’') & 2002 & 50.3 & 38106 & 9 years & -10 & Hypertension \\
\hline 010 & 46 & 400 (5'3') & 2005 & 70.8 & 38109 & 13 years & -18 & Hypertension \\
\hline 011 & 40 & 270 (5'8') & 2011 & 41 & 38109 & 7 years & +70 & Hypertension \\
\hline 012 & 42 & $237\left(5^{\prime} 6^{\prime \prime}\right)$ & 2000 & 38.2 & 38109 & 3 years & +33 & $\begin{array}{l}\text { Hypertension, nerve } \\
\text { damage }\end{array}$ \\
\hline 013 & 47 & 197 (5’9’') & 2006 & 29.1 & 38109 & 2 years & +10 & Hypertension \\
\hline 014 & 47 & 254 (5'6”) & 2005 & 41 & 38109 & 15 years & -10 & $\begin{array}{l}\text { Hypertension, heart } \\
\text { disease }\end{array}$ \\
\hline 015 & 38 & 143 (5'3’') & 2009 & 25.3 & 38106 & 18 years & -85 & High cholesterol \\
\hline 016 & 48 & $193\left(5^{\prime} 5^{\prime \prime}\right)$ & 2008 & 32.1 & 38106 & 3 years & -9 & Bipolar disorder \\
\hline 017 & 44 & 212 (6'1"') & 2005 & 28 & 38109 & 3 years & -28 & Heart disease \\
\hline 018 & 45 & 220 (5'6”) & 2000 & 35.5 & 38109 & 8 years & -30 & Neuropathy \\
\hline 019 & 42 & 227 (5'8’') & 2001 & 34.5 & 38109 & 15 years & -30 & $\begin{array}{l}\text { Hypertension, high } \\
\text { cholesterol }\end{array}$ \\
\hline 020 & 48 & 195 (5’2”) & 2003 & 35.7 & 38109 & 5 years & -85 & Hypertension \\
\hline
\end{tabular}


Table 4-2. Study population statistics, overall sample.

\begin{tabular}{lcccc}
\hline $\begin{array}{l}\text { Average age in } \\
\text { years (SD) }\end{array}$ & $\begin{array}{c}\text { Average BMI } \\
\text { (SD) }\end{array}$ & $\begin{array}{c}\text { Average length of } \\
\text { time in ZIP in } \\
\text { years (SD) }\end{array}$ & $\begin{array}{c}\text { Average } \\
\text { cumulative weight } \\
\text { loss/gain in lbs. } \\
\text { (SD) }\end{array}$ & $\begin{array}{c}\text { Average time } \\
\text { since diagnosis } \\
\text { (SD) }\end{array}$ \\
\hline $43.35( \pm 3.6)$ & $36.82( \pm 11.6)$ & $10.25( \pm 10.8)$ & $-20.7( \pm 43.2)$ & $8.95( \pm 3.43)$ \\
\hline
\end{tabular}

Table 4-3. 38106 study population statistics.

\begin{tabular}{lcccc}
\hline $\begin{array}{l}\text { Average age in } \\
\text { years (SD) }\end{array}$ & $\begin{array}{c}\text { Average BMI } \\
\text { (SD) }\end{array}$ & $\begin{array}{c}\text { Average length of } \\
\text { time in ZIP in } \\
\text { years (SD) }\end{array}$ & $\begin{array}{c}\text { Average } \\
\text { cumulative weight } \\
\text { loss/gain in lbs. } \\
\text { (SD) }\end{array}$ & $\begin{array}{c}\text { Average time } \\
\text { since diagnosis } \\
\text { (SD) }\end{array}$ \\
\hline $42.4( \pm 3.8)$ & $34.8( \pm 10.4)$ & $12.2( \pm 13.3)$ & $-29.6( \pm 40.5)$ & $8.1( \pm 3.29)$ \\
\hline
\end{tabular}

Table 4-4. 38109 study population statistics.

\begin{tabular}{lcccc}
\hline $\begin{array}{l}\text { Average age in } \\
\text { years (SD) }\end{array}$ & Average BMI (SD) & $\begin{array}{c}\text { Average length of } \\
\text { time in ZIP in } \\
\text { years (SD) }\end{array}$ & $\begin{array}{c}\text { Average } \\
\text { cumulative weight } \\
\text { loss/gain in lbs. } \\
\text { (SD) }\end{array}$ & $\begin{array}{c}\text { Average time } \\
\text { since diagnosis in } \\
\text { years (SD) }\end{array}$ \\
\hline $44.5( \pm 2.59)$ & $39.3( \pm 10.36)$ & $7.89( \pm 4.93)$ & $-9.78( \pm 41.44)$ & $10( \pm 3.29)$
\end{tabular}




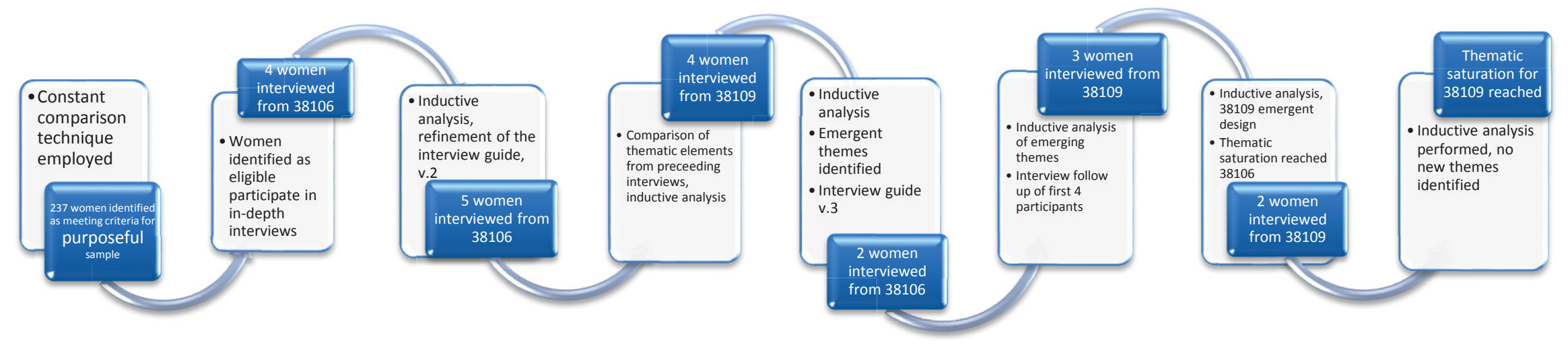

Figure 4-1. Workflow of recruitment and constant comparison throughout the data collection process. 
Thematic saturation was reached for women in 38106 after 11 interviews; saturation of thematic elements was reached after 9 interviews from women in 38109.

This Chapter will be divided into four parts. Part 1 will provide an overview of general themes found in the data as identified by Tables 4-5 and 4-6; Part 2 will address the research questions specifically, and provide an analysis of each theme in the context of questions 1 and 2. Part 3 will address research question 3 and analyze GIS data within the context of relative themes identified in Part 1. Part 4 will analyze identified differences as observed between residents and environments of the 38106 and 38109 ZIP codes to better understand the dynamics occurring between similar demographic areas with different environmental circumstances.

\section{Part 1: Identified Major Factors Affecting Weight Loss, Physical Activity, and Health Maintenance}

Part 1 of this results section details several themes identified among the study population (in both zip codes) as perceived encouraging and discouraging factors toward successful and maintained weight loss, positive health maintenance, and physical activity. A summary of factors identified by category are listed in Tables 4-5 and 4-6. With regard to identified behavioral and environmental positive influences on weight loss and health maintenance, overarching main themes identified among the overall study population included (1) nutrition; (2) medications; (3) physical activity and exercise; (4) community and aspects of social environment, and; (5) community and aspects of the physical and built environment. Several dimensions are associated with each overarching theme. Major negative influences regarding weight loss, physical activity, and health maintenance included (1) nutrition; (2) factors related to medications; (3) inconsistent physical activity and exercise; (4) community and aspects of social environment; (5) community and aspects of the built and physical environment; (6) psychological influences; and (7) economic factors. Similarly, the main factors identified incorporate several associated dimensions.

In part 2, the research questions are specifically addressed. Many pertinent themes were identified in the analysis that were not the focus of the research questions; these themes were discussed in Part 1 of the Results section. Themes that have been discussed in the previous section will not be restated in Part 2 of this reporting; however, attention to these items will be called upon as appropriate.

A major thematic element not addressed specifically by the research questions, but was found to be relevant and substantially affected factors relating to weight loss, health maintenance, and physical activity among the women of both ZIP codes included psychological considerations of the women interviewed. Psychological influences, defined as how participants mentally oriented themselves and coped with their diabetes circumstances, were identified as affecting weight loss and health maintenance and were indirectly gauged from participant responses. 
Table 4-5. Factors that positively influence health status and weight loss, and dimensions.

\section{Factors identified as positively influencing health status and weight loss}

Nutrition

Medications

Physical activity and exercise

Community and aspects of social environment

Community and aspects of the built and physical environment

Factors associated with individual outlook

Other factors
Dimensions identified

Decreases in fried food consumption

Increases in water, juice, milk, etc. consumption

Increases meal frequency and the adoption of smaller portion sizes

Increases in vegetable consumption

Decreases in carbohydrate consumption (e.g. spaghetti, potatoes)

Increases in baked poultry and pork consumption

Attributions of metformin contributing to accountability to nutrition modifications

Consumption of diet pills attributing to weight loss

Walking

Chores

Utilization of fitness facilities

Aerobics and group fitness classes

Walking within the home

Fitness DVDs

Close knit neighbors within the neighborhood

Neighborhood watch

Law enforcement presence

Other's presence during engaging in physical activity

Older neighbors within the neighborhood

Mobile farmer's market

Farmer's market

Parks

Proactive/positive/disciplined attitude regarding habit change

Fear of amputation and side effects via observations of others Attending diabetes management classes 
Table 4-6. Factors that negatively influence health status and weight loss, and dimensions identified.

Factors identified as negatively influencing health status and weight loss

Nutrition

Factors related to medications

Inconsistent physical activity and exercise

Community and aspects of social environment
Dimensions identified

Snacking with easy access foods (e.g. potato chips)

Soda and Kool-Aid consumption

Continued consumption of carbohydrates despite healthcare advice otherwise (macaroni and cheese, cake)

Continued consumption of high fat content containing foods despite advice otherwise (e.g. fried chicken, fast food hamburgers)

Inability to sufficiently plan healthy meals due to life responsibilities; perception that family will not enjoy nutrition modifications

Nonadherence to metformin

Nonadherence to insulin

Nonadherence to medications related to additional comorbidities indicated (e.g. hypertension medication)

Perception of insulin contributing to weight gain

Difficulty exercising due to comorbid conditions

Work and job responsibilities

Poor patient/provider relationship

Presence of neighborhood crime (youth crime, prostitution, drugs, gangs, murder rates, safety concerns)

Generational diabetes

Perceived lack of family support

Inactive neighbors

Perceived social norms (e.g. nutrition) 
Table 4-6. Continued.

Factors identified as negatively influencing health Dimensions identified status and weight loss

\section{Community and aspects of the built and} physical environment

Individual outlook considerations

Economic factors

Other factors

\author{
High numbers of corner stores \\ Inadequate grocery store merchandise \\ Lack of sidewalks \\ Limited access to fitness facilities \\ Perceived unsafe parks \\ Limited access to quality produce \\ Lack of diabetes targeted and education resources
}

Present orientation - present takes precedence over future Effects of stress on health considerations and consistency Management disappointments leading to frustration and resignation

Perception of the effects of aging

Perceiving weight loss to be difficult

Depression

Perceiving change to be difficult

Lack of motivation

Medications and medication equipment costs

Lack of health insurance and perception of poor quality providers

Limited financial resources to obtain recommended diet

Limits in residential options

Lack of education and knowledge regarding diabetes

Weather

Substantial presence of stray animals that deter walking and outdoor physical activity 
These considerations included (1) a present "today" orientation rather than a "tomorrow" perspective; (2) substantial stress levels due to financial and social considerations; (3) management disappointments that discouraged further attempts to lose weight, and the perception of weight loss being difficult; (4) a general lack of education regarding how to lose weight and maintain health adequately; (5) depression and resignation, and; (6) the perception of "change" being difficult. Many of these circumstances appeared to interact with other factors that affected the ability to lose weight, including degree of encouragement available through community and social support, as well as economic factors. Additionally, a few women who reported success appeared to have a more positive and proactive undertone in reporting their experiences. Perceived positive experiences were observed to be associated with more perceived control over the experiences:

"I feel great about [losing weight] because I'm putting my mind to something that I wanna do on my own, and nobody is forcing me or telling me how, you know, I take my time doing it. I feel great about it." - Participant 002

Another woman stated:

"Well I went to the doctor yesterday and I weigh 230 now, because I've lost 9 pounds, so I'm sticking to my diet and I'm exercising like I'm supposed to now. At first it's kind of hard because you don't want nobody telling you nothing." Participant 004

A third woman discusses how although healthcare advice suggests that she diet and exercise, she has to be motivated enough to want to follow the advice and take care of her health:

"I gotta motivate myself. I mean, [not] just you telling me to do something, I have to want to do it myself...I just try to maintain, and do what I gotta do to make myself feel better. Like I said, can't nobody make me - motivate me, the doctor can tell me all day long you need to exercise, you need to do this here, but exercising, and dieting and all this, it ain't no easy job." - Participant 009

A common sentiment expressed among the women was how difficult it can be to plan for the future when the present seems to be most important. Particularly, several women expressed that they had present circumstances that required attention at that time, especially when framed from an economic perspective, or from a perspective that characterized stress, depression, and resignation to circumstances. For example, one woman from 38109 stated:

"I got a lot going on right now...I'm consumed with debt and a lot of other stuff... [my health priorities] are not where it should be, but I do it, but it's not where it should be." -Participant 011 
Generally, the more stressed or resigned women were to their circumstances (circumstances including environmental, familial, personal health, and residential conditions), the less likely she appeared to be consistent in establishing a regimen geared toward weight loss and health maintenance. Depression, resignation, and stress characterized the frame through which many of the participants indicated their difficulties in losing weight. One participant characterized the difficulty she had following a healthy regimen through the stress. She said:

"I could eat what I wanted to eat but, it was like, lessening up on my carbs and stuff like that, knowing how to eat my carbs, and I did it for a while, and I did lose weight, and I kind of got stressed out a little bit, a little depressed, and I kind of gave up...it was to a point where I was like I'm gonna eat what I want to eat, I'm not gonna worry about it, you know, I was basically giving up. But then I noticed that after I was trying to give up it was still affecting me... I stopped because I said I'm not just going to worry about it. I was giving up, I was just tired. I was like if I eat it I eat it, it was like whatever." -Participant 009

One participant in the study was particularly proactive about her health. Previously living in 38106 for 18 years, she had since moved to a new location that she perceived as more safe, more of her neighbors were actively engaged in physical activity, and as a result she had become more health-aware. She also was able to frame her diagnosis as a positive experience:

"So really, [being diagnosed with type 2 diabetes] came to me as a negative in all, it's a blessing, because I'm reaching my weight goal, I'm more cautious about what I eat and how I take care of myself, and I see things, like for instance fruit, I pick better choices. Now some days I'll have that white powdered donut episode and I may buy a big bag, and I'll eat 4 and to keep myself [from overindulging] I'll give them away. But I learned that you know what, I'm not that old where I can't change my eating habits, so it helps. -Participant 015

Counter to what was typically reported by women who have trouble with nutrition accountability during preparation of family meals, this particular participant refused to cook separate meals and instead, encouraged her husband to eat what she ate. She acknowledged a sentiment that may underlie women preparing two meals for themselves and their families. She reported that women may believe that eventually, they can return to previous nutrition habits once the diabetes condition is adequately managed; however, this participant acknowledged that this is an inappropriate mindset:

“...when I officially became a diabetic, I didn't cook two separate meals. I didn't cook a meal for him and a meal for me. Because that's going back into I can relapse to that. You ate what I was going to eat. He would complain about salt and I said put your own salt on that. We're sticking to the plan. And I'm not about to kill myself trying to make you happy. If you wanna eat fast food, you eat it, brush your teeth, and come back, but you're not gonna set me up where I cook two separate meals. And that's the wrong mindset because you're teaching 
yourself that eventually, you're going to go back to eating what they eat." Participant 015

She also talks about her healthcare experience and taking control of her care, which further demonstrates her positive psychological framing in managing her diabetic condition:

"Like I went to the Medplex, and I took off my shoes, and my socks, because you're supposed to examine my feet. So this resident doctor came in and he said what are you taking your socks off for? And your shoes? I said because I need you to look at my feet. He said what's wrong with them? I put my feet in his hand, and I said open up your hand, put those gloves on and open up your hand, I laid my feet there. He said, you got rings on your toes, and I said I sure do, and I paint my toenails...I'm a diabetic, he's looking at me like okay...I said you make sure I do not have any cuts or sores on my feet. You're supposed to be telling me this. So when the doctor came in, my feet were still there, and he said well I see you're getting good care. I said no, I'm not! I told him to sit down, put some gloves on, and I put both my feet [in his hands], you're gonna check my feet. And he said why? And I said because if I'm numb in that foot, and I go out here and hurt myself, you're gonna - they're gonna cut my toes off...up to this day, that doctor checks my feet! When I got to the doctor every three months, I'm so excited when he's gonna tell me he's gonna take me off of something." Participant 015

When asked what prompted her to become more aware of her health, she indicated that her diagnosis with type 2 diabetes was a turning point for her. After diagnosis, she moved into a new environment and became more aware of healthy habits, and fostering a healthier lifestyle.

Psychological influences, particularly stress, depression, and resignation, appear to complexly interact with many of the other factors that affect weight loss, physical activity, and health maintenance. However, amongst the women, social support appears to have a diminishing effect on the amount of stress observed among the women, which appears to increase health maintenance consistency and weight loss effort. For example, one woman states the following about the effects social support has on her stress levels, contributing to her motivation:

"Because I have people to motivate me and keep me on the right track with that. Because sometimes you get thrown off, and you get frustrated from people try to help you, and you don't realize that you're running your help off when they're here to help you, but sometimes you get frustrated because you get tired, you don't want to do it, but I get frustrated sometimes when I really don't want to, but I still have to keep myself motivated and keep on going." - Participant 004

Overall, several factors were identified that were perceived to both encourage and discourage physical activity and health maintenance among the study participants. There 
were generally more discouraging factors reported by women within the context of this study; however, encouraging factors including park availability and the importance of social support were reported to be key to general consistency despite external and internal circumstances.

\section{Part 2: An Analysis of Research Questions 1 and 2 in the Context of Identified Themes}

The following presents research questions 1 and 2:

1. What are the methods weight loss employed by low-income, African American women with type 2 diabetes mellitus in the 38106 and 38109 ZIP codes of Memphis, TN?

2. What are the sociocultural, economic, and environmental influences that encourage or discourage successful weight loss, health maintenance, and physical activity among low-income, African American women with type 2 diabetes mellitus in the 38106 and 38109 ZIP codes of Memphis, TN?

\section{Weight Loss Strategies Employed by the Study Population}

Women in both 38106 and 38109 expressed using similar strategies in their attempts to lose weight. The main categories identified in weight loss and health maintenance attempts were (1) nutrition modifications; (2) physical activity and exercise; (3) medication utilization, and; (4) diabetes management classes.

\section{Weight loss strategies - Nutrition}

With regard to nutrition, important overall factors reported to contribute to successful weight loss and health maintenance included (1) decreases in fried food consumption; (2) increases in water, juice, and milk consumption; (3) increases in meal frequency and adopting smaller portion sizes; (4) increases in vegetable consumption; decreases in carbohydrate consumption, and; (5) increases in baked poultry and pork consumption.

Nutrition was identified by all of the women in the study to contribute to weight loss and health maintenance; every participant in the study mentioned that they have, at some point, attempted to change their habits and eat more healthily since type $2 \mathrm{DM}$ diagnosis. Perceived consistency in these behavior changes were demonstrated to contribute to having engaged in successful weight loss efforts among the participants. Even when no formal diabetes education classes were taken, the study population generally reported an understanding that consuming carbohydrates was not conducive to 
health and weight loss goals. Many women acknowledged that this advice most often was given by their health care provider.

One participant related making dietary changes, including baked chicken and pork. She also attempted to eat low carbohydrate containing chips, yet initially there was no effect. However, with additional modifications, including eliminating Kool-Aid and soda, and consuming more water, she lost weight and is maintaining the weight lost:

“... well it's when I give up all my fried foods and I started to eat baked chicken, baked pork chops, just baked food, but I'd still be snacking on the side, like chips and I would try to get the low fat, low carb chips (inaudible) but still I could not lose weight... I had a breakthrough a few months ago and I just started drinking more water and not drinking kool-aid and diet pop, even though diet pops don't have sugar in it, still - to me it's still calories I don't know, so I started drinking more water, and I finally had a breakthrough at the beginning of this year, and I'm - now I'm not losing weight, I had lost 10 pounds, but I'm maintaining the same weight now, I'm not gaining any, I'm not losing any, but I'm not gaining any either." - Participant 001

Although nutrition was cited as contributing to weight loss among many of the women, most also indicated that making these nutrition modifications consistently was considerably difficult to accomplish. Continued snacking and soda consumption were mentioned frequently as difficult to limit within their daily lives. Additionally, limiting carbohydrates, which were reported by the women as perceived as contributing the most to weight gain, particularly seemed to be difficult. Some described this struggle as being one where they simply had to know what to eat and what not to eat; however, more often than not this was easier said than done. Progression in eliminating poor choices and establishing good habits was stepwise - once one result had been established, others were easier to implement. Establishing nutrition habits without reverting to previous habits was a concern among many of the women.

One study participant summarized this overall sentiment:

“...I actually used to eat a lot, and then after that, once I started eating the small meals, it was like my stomach adjusted to them. I started getting full faster so I would want nothing but small meals. And basically then I cut out soda, I cut sodas out, I basically just drank water, and some juices or milk or something, and I cut down on my bread intake, but basically I just started eating smaller portions... I was choosing better, because I knew that, well I wasn't a big sweet eater no way, but I found that diabetes didn't come from sweets, it came from starches turn into sugar, fat, (sic) so I cut out a lot of that too because I did used to like spaghetti, I loved creamed potatoes, so I cut out some of those types of foods, that turned to sugar fast, so. I ate more vegetables and fruits, stuff like that." Participant 008 
Nutrition modification difficulties were also connected to many other factors that were reported to negatively influence weight loss and sufficient health maintenance, including social support, difficulties in planning and eating the "right" foods due to life circumstances (right foods being defined as fruits, vegetables, carbohydrate limited foods, and consuming water, and low-sugar beverages), economic considerations, stress, accessibility to healthy foods, and self-discipline. One dimension of social support related to nutrition was relayed by some women as the inability to sufficiently plan healthy meals due to the perception that their families didn't enjoy the nutrition modifications made. As a result, women would either have to prepare two separate meals, forgo preparing meals for their families, or make one meal that adequately addressed their nutrition concerns with complaints from their family. This was implied to be a sacrifice to their own nutrition requirements, as they would cook foods that were perceived as nutritionally insufficient to maintain their health in order to keep their families happy. One woman's sentiments regarding cooking for her family had undertones that seemed to imply that the nutrition requirements which could assist in controlling her diabetic condition were undesirable for both her and her family:

"Yeah they eat the same things I eat. That's probably another reason why, because I try to cook them, not trying to feed them all the stuff I know I need to eat but, I don't know."- Participant 012

Also relating to the social support dimension of nutrition, one woman described how she made necessary changes to her diet and she also extended these changes in her cooking with her family. However, her family generally does not support the changes she has made in her prepared meals for them, and although she has attempted to maintain these changes, pressure from her family makes sustaining these changes more difficult for her: The respondent also highlights how modifying family nutrition to incorporate healthier options can be difficult:

“...my husband, he just wasn't gung-ho about it, trying to change eating habits, or lifestyle, not using grease or oil, and using you know, olive oil, because they think the food tastes different, and funny...I had to persuade them, but my daughters, they go to my mama's house, who believes in putting fatback meat in a pan, and putting it on top of the refrigerator and letting it sit for months, so that the meat can - so that the sauce and soak up into the meat and put it in her greens and it goes through her greens that she already salted with salt, they love that...they think I deprive them of food. But even with my mother she says you grew up eating like this, and my whole family ate like this, and [I say] your whole family has had high blood pressure... or are fighting diabetes. You know, I have an aunt, the same aunt I was referring to earlier that also has diabetes, she - before she cooks her meal on Sunday or whenever she's cooking, and the cake will be cooked first. She's probably eating it by herself." - Participant 017

The previous quote specifically shows how engrained nutrition habits can be within families, contributing to nutrition's cultural influence on health maintenance, 
difficulties with losing weight within the African American community, and potentially contributing to generational diabetes.

Another dimension of nutrition modifications and difficulties was expressed predominantly among women who had demanding work schedules that did not allow them to eat frequently or in a healthy manner. An example of a rigorous meal schedule that produced positive results for one woman is listed below, although she relates that she has been very inconsistent with keeping this schedule:

"When I would eat baked food, I had a little schedule going on, like for instance, I ride around, I'm a CNA, so as I ride around...I would get me a grilled six inch chicken sandwich, a Dr. Pepper, and I might get a bag of chips to go with it. So I would go to a restaurant on like - like morning time, I would try to eat my breakfast in the morning. I would do like my little wheat toast or the bagel or I used to eat the turkey bacon and one egg, I'd try to make sure that everything was in the right amount of carbs. Then morning I'd get [home] between 9 and 10 and I'd eat something like the peanut butter crackers or peanuts or something in my car that I could nibble on until lunchtime. I was doing all of that and I lost weight and everything!" - Participant 009

Although nutrition was commonly indicated as a crucial key to weight loss, at times it seemed to undermine most women's ability to lose weight, particularly relating to their willpower, social support received, and breaking established poor habits. Some women indicated that this may be due to what was commonly observed of their peers (relating to social influences), as well as limitations as to what is readily available for consumption in the community. One woman discussed the difficulty she has taking care of herself because of what she observes of her neighbors' consumption habits, and how being around people who consume counterproductive foods for her condition can have a detrimental effect:

"[It's difficult to take care of myself] because they don't eat nothing but junk around here...I have to shop for myself [in a healthy way] because the people that I live around, they don't even think [about] fruits and vegetables and stuff like that. It's all about chips and cookies. So, I have to keep myself motivated on that, I have to pray about it and keep myself on track. Because being around people that don't do it, it'll have a big effect on you." - Participant 004

Overall, nutrition appears to both negatively and positively affect weight loss and health among the study population. Although generally, women report that they can and have made necessary modifications to aid in weight loss, it is reported to most often be difficult mentally, financially, and logistically to maintain nutrition and dietary changes that are conducive to effectively managing diabetes and losing weight consistently. 


\section{Weight loss strategies - Exercise}

Exercise was another primary strategy employed by women, through various means. Methods used in physical activity encompassed both home and outdoor physical environments and included (1) exercise via walking around the neighborhood; (2) walking in parks; (3) chores; (4) aerobics and exercise classes, and; (5) exercising within the home. Women in 38109 perceived lack of sidewalks as a barrier for physical activity and thus tended to employ means of physical activity that did not include walking around the neighborhood. Employing methods of physical activity other than walking in the neighborhood was also associated with the perception of an unsafe neighborhood, along with the perceptions of the absence of sidewalks in the neighborhood. Modes of physical activity employed are relayed in the following excerpts:

"Well, I walk every day, and I know I can't get too - too fat, I know I have to try to watch what I eat and keep my weight to a, you know - I try to stay between 140 and 150 anyway, but really, I...the doctor told me I need to be between 127 and 130 for my height...I got pumped up to go to the gym, you know, riding that bicycle thing that [rides] in place and stuff..." - Participant 001

One woman describes her experience with aerobics:

"...now I do aerobics, low-impact aerobics...I have [an aerobics] tape at the house...sometimes I'd walk, some days I walked, like if it's nice I'll walk and if not I'd do aerobics..." - Participant 008

Another study participant relays her strategy of weight loss through chores:

“And if I didn't walk, I would cut the grass because they would help me boost up my energy. I cut grass [because] when I talked to the doctor he said that helps out too. It works on your heart." -Participant 009

An additional dimension of a process utilized to engage in physical activity included physical activity at work, with one woman reporting the following:

"I do a lot of walking at work, at my night job, I climb stairs, and I try to do a little workout in the morning-time. It's a DVD." - Participant 019

\section{Weight loss strategies - Medications and effects on weight loss and health maintenance}

Factors related to medications taken by study participants were perceived to be influential in both providing accountability aiding in weight loss, and contributing to difficulties with losing weight. Metformin was the most frequently referenced medication taken for type $2 \mathrm{DM}$ by study participants. Most women who took this medication suggested that to a certain extent, the medication held them accountable 
nutritionally although it remained difficult to lose weight. Women report that one of the effects of metformin is stomach upset, especially when high fat-containing foods are consumed. One woman states:

"Okay, with me dieting, everything was digesting and everything was doing the right thing. But once when I still was taking [metformin], still eating what I want, it still was making me still be big and I still was feeling nauseated, I was very nauseated when I wasn't eating the right foods, and then I still took the medication." -Participant 008

Insulin was another commonly referenced medication taken by the study participants. One woman who reported mental fatigue with the process of maintaining her health and difficulty with nutrition modifications indicated that she preferred to take insulin to keep her blood sugar under control, despite failing to make necessary diet changes. She was not necessarily concerned with losing weight, although she did state that ideally she would like to. She stated that rather than change her diet, she preferred to take insulin instead. This particular respondent also had perceived limited social support, was going through a divorce, and experienced substantial familial diabetes. She states:

"I even tried to get back on insulin, they wouldn't let me get on it. They told me just take my pills, and eat right, check my sugars and but, they won't let me get back on it. I wanted to, because my sugar was just out of control. And no matter what I ate - I guess I wasn't eating the right things I probably was eating stuff I wasn't supposed to eat, like spaghetti, and a lot of starch, because I like macaroni and cheese, and mashed potatoes, and [sigh] they probably try to tell me to cut back but it's hard." - Participant 012

Some women indicated, however, that taking insulin made losing weight significantly more difficult because they perceived that it may have contributed to weight gain. This perception, along with a poor health provider relationship, was noted to be a significant determinant of weight loss success for one participant. Concern for regulating blood sugar levels, along with extending seemingly contradictory advice to lose weight can lead to frustration, which was a factor seen among many women in the study. One woman, who eventually took her health into her own hands and began to become proactive with her healthcare provider, shared her experience on figuring out why she couldn't lose weight, and her perception that insulin was counterproductive to health goals:

"And I said [to my doctor] y'all tell people they have diabetes, lose weight, lose weight, lose weight, but y'all do not understand that the insulin is what makes some people gain the weight, which it was making me gain weight. That was my biggest problem. I was on two different kinds of insulin, I was taking NovoLog during the day and I was taking Levemir at night. The insulin was blowing me up." - Participant 020 
In addition to taking metformin and insulin for blood sugar control and taking metformin for weight loss, four women indicated that they also took diet pills to aid in losing weight. However, all of the women that reported this also indicated that they were inconsistent, and that when consumption of pills stopped they gained any weight back that was lost. One woman describes her experience with weight loss using diet pills:

"The only way that I would really gain my weight back, and say I just don't care, is when I don't. See, it's a difference. When you take these right here [points to metformin], you don't eat that much. But then when you miss them, that's when the weight comes back." - Participant 006

Although medications prescribed for insulin are generally targeted toward better A1C level maintenance, women reported difficulty losing weight while taking insulin. This was perceived to be counterproductive to weight loss by the women, which in turn contributed to communication breakdowns between health care providers and the women. One woman relayed how extensive the communication breakdown was between her and her healthcare provider:

"You know what [the doctor] told me? He said if you ever have to go to the hospital, do not tell them that I'm your doctor... because he felt like I wasn't doing what he was telling me to do, and I was [doing what he asked]." Participant 020

This was especially relevant when there is limited knowledge regarding healthy and productive behaviors, along with frustrations concerning lack of progress.

\section{Weight loss strategies - Attending diabetes management classes}

Study participants also reported attending diabetes management classes as a way to maintain their health and gain knowledge to more consistently control blood sugar levels. Women who took management classes usually indicated that it helped them learn how to better manage their nutrition, helping them lose weight more effectively. Classes were referenced as not only low in cost, but also contributing to accountability.

Although several women indicated that they had taken management classes, one woman shared how taking classes has allowed her to improve various facets of her life:

"It's a program for people that [have] diabetes or for anyone [that has] obesity and wants to lose weight, it's right here on Madison. It's like an exercise class, they have diabetic classes for eating, they'll show you how to eat and everything. Yeah, I started trying to lose weight, I signed up for Hope and Healing and they told me to start going to the classes, start going to the food classes where I can learn to cook for myself, so since I've been doing that I've been doing a lot better, I sleep better at night, I rest better, well, one time they said I was have, like, I was 
losing my breath when I sleep at night, so I started losing weight and stuff and she said I don't even have to go through that class anymore..." -Participant 004

Generally, due to logistic and economic factors, the women in the study tended to utilize means of weight loss that were both low cost and easily accessible. Nutrition modifications were the easiest and most accessible method of weight loss reported by the women, facilitated by diabetes management classes. However, nutrition modification was also the most consistently reported to be the most difficult change to make. Difficulties encountered with engaging in regular physical activity included work schedule, a lack of places around the neighborhood to engage in regular physical activity, perceived unsafe environments, and the absence of accountability. When these difficulties were encountered among the study population, women reported being more inconsistent with physical activity effort. Similarly, difficulties were typically met with resignation or reduced concern for health maintenance.

\section{Sociocultural Factors that Encourage and Discourage Weight Loss and Health Maintenance}

The goal of examining sociocultural influences was to assess the perception of established lifestyle patterns, social, and cultural influences, and how these potentially affect health maintenance, physical activity, and weight loss among low-income African American women from two identified ZIP codes in Shelby County. Two key themes were identified as pertaining to sociocultural influences that encouraged the perception of weight loss and engagement in physical activity among the participants. These include (1) modification of established nutrition patterns and (2) generationally/socially passed information. Discouraging factors identified within the study population that incorporated aspects of sociocultural influences included (1) generational diabetes, (2) nutrition patterns, and (3) area crime.

\section{Sociocultural factors - Nutrition}

Nutrition effects on the study population is a multidimensional and complex factor that was intricately woven into many aspects of participant lives. It was identified as dominant sociocultural factor that was potentially negatively affecting adequate health maintenance among the study participants. Specifically, nutrition was acknowledged as a factor that may contribute to social cohesion within the community and family; however, the knowledge of foods consumed and their effects on adequately managing their diabetes was limited. Most women demonstrated that they generally understood that fruit and vegetable consumption should increase, as conveyed to them by their healthcare providers; additionally, many women also had knowledge about the effects of carbohydrates such as spaghetti, and its effects on blood sugar management. However, when asked about methods of change and how they maintained consistency with healthcare provider recommendations, most women demonstrated some difficulty. For 
example, one woman demonstrated the importance of eating framed in a sociocultural and psychological context:

"I was the type of person that would eat until I go to bed. Eat 'cause I'm depressed. Eat because somebody mad at me. Eat because it's a cake. Eat because it's Christmas. Eat because it's Thanksgiving. Eat because someone having a baby. Eat because somebody got married. It don't matter what you eat, just eat!" - Participant 017

\section{Sociocultural factors - Generational diabetes}

Once knowledge was acquired from varying sources (including healthcare providers and management classes), consistency was a limiting factor in whether or not this group of women was successful in losing weight. Consistency in maintaining health changes was primarily reported to be through social accountability, including family members who also possess diabetes. Reported extensive histories of familial diabetes, socially observed behaviors, and passed-down information was identified both an encouraging and discouraging factor in weight loss and physical activity patterns; however, reported perceptions were primarily more discouraging in nature. One example of an extensive family history of diabetes was recounted by one participant:

"My whole family is diabetic. My mom, my grandmom, my auntie...my mom is a bad diabetic...my grandma's sisters had it, she had it, both my aunts have it, my sister has it, my cousins have it, not all of them, but you know...basically my mom is the one that encourages me...It's probably our eating habits too because my great great grandma had [diabetes], and she didn't know that back then, they didn't diagnose that, one of her legs stayed swollen all the time, and that's one of the things with diabetes, your limbs swell, so your sugar is raised, so I think she had it, but she didn't know she was sick like that... I'm trying to lose this weight. I'm trying to lose 50 pounds by the end of summer or the beginning of winter, and I'm going to try to keep that off, and try to still maintain - I don't think that once the weight, I don't think that sugar's gonna go away. My mom is so little and it hasn't went away. So I'm just gonna try to keep it under control so I can have my legs and my feet and my toes. And my eyes and my kidneys - I just wanna keep those until I become an old woman..." -Participant 019

Positive external accountability was mentioned by eight women as contributing to their weight loss and health maintenance efforts. Many women reported family members, neighbors, parents, and friends holding them accountable in terms of nutrition modifications. However, most of these accountability persons were also people who themselves possessed diabetes. For example, one woman states:

"So [my family] sits down and kind of discuss about the food products, and the exercise and, what they talk about to they doctors (sic), and what I talk about to 
my doctor and I'm like he told me the same thing, [they're like] yeah they told me to do the same thing." - Participant 002

The extensive generational effects of diabetes observed among the respondents included passed down poor health behaviors, observation of family experiences with diabetes, and counterproductive expectations of diabetes effects due to observations of family members. Only 4 women in the study population reported that they did not have familial diabetes references to their knowledge. This dimension of socio-environmental influences was identified as discouraging in the perception of weight loss, health maintenance, and physical activity patterns. Several women indicated extensive familial and community social ties who also were affected by diabetes, and where more often than not, family members and friends did not adequately and effectively manage their health. Women cited that most of their friends were diabetic; some had both parents possessing the condition; some had siblings, aunts, uncles, and cousins, who had the condition. Many had parents who died from the disease, who had significant complications, including amputation, and this gave them access to firsthand knowledge of its potential consequences. Even though women understood this to be the case, many women still found it difficult to maintain their health. One woman discussed how having family and friends extensively affected by diabetes contributes to her perception of the difficulty of losing weight:

“...Most of my friends are diabetics too so, I have a brother-in-law, he's a diabetic too so basically I see how it really feels to be like that, losing all the weight kind of hurt, but I'm dealing with it...it's hard to lose weight." -Participant 002

In some ways, expectations of the disease preceding diagnosis, and expectations after diagnosis due to familial references undermined efforts to maintain health, particularly if family members were unable to consistently do so. Also observed in women's reports of generational diabetes was with expectation of diagnosis and disease seemed to come a degree of resignation and expectations of failure in regards to attempting to alter the course of the disease. Additionally, the more extensive the proximal diabetes influences, the more likely women were to report perceiving not being in control of their circumstances.

One woman also discussed how it had been difficult for her to maintain her health because of a general lack of information that had been generationally established. Further, she perceived that weight loss cannot necessarily resolve the condition reported losing weight for other reasons that included maintaining her eyesight, reducing the amount of comorbidities that she possesses, and general mobility. She states:

"It's probably our [family's] eating habits too because my great great grandma had [diabetes], and she didn't know that back then, they didn't diagnose that, one of her legs stayed swollen all the time, and that's one of the things with diabetes, your limbs swell, so your sugar is raised, so I think she had it, but she didn't know she was sick like that... I'm trying to lose this weight. I'm trying to lose 50 
pounds by the end of summer or the beginning of winter, and I'm going to try to keep that off, and try to still maintain - I don't think that once the weight [comes off], I don't think that sugar's gonna go away. My mom is so little and it hasn't went away. So I'm just gonna try to keep it under control so I can have my legs and my feet and my toes. And my eyes and my kidneys - I just wanna keep those until I become an old woman." -Participant 019

An interesting dynamic occurred between women's knowledge about the disease and its effects, and their willingness to circumvent the effects through present changes. Many women, as indicated previously, have extensive family histories of diabetes. Many report observing the deleterious effects of diabetes on family members, including amputations, strokes, and death. However, many women also indicate that although they are aware of this dynamic, they are still unable to make consistent health changes. For example, one woman indicated that although her father recently had died from diabetes complications, this was not sufficient enough for her to consistently change her diet; this characterizes many of the responses giving reasons why consistent changes are difficult to make. Additionally, this respondent had an expectation that she would get the disease before she was diagnosed with it. She states:

"Well in a way I kind of knew that it was gonna come because my dad died from it, so, me and my oldest sister got it, so um, once we turned over 40, go in our 40s that's when it happened, but I deal with it it's okay, I can deal with it." Participant 003

\section{Sociocultural factors - Crime}

Neighborhood crime was a sociocultural-related discouraging factor particularly among the 38106 resident participants (although the perception was expressed among 38109 residents as well) and was associated with discomfort with engaging in physical activity within their neighborhood. Women were concerned for their safety. Women particularly in 38106 acknowledged the social culture of their environment and were aware that the general crime rate in this area is high. Largely, crime and the threat of bodily harm seemed to overrule engaging physical activity outdoors, particularly if the women were not from the area. The assumptions of area crime were expressed as discomfort with walking alone and was primarily reported by women who had limited ties in their neighborhoods, or had been in their environments for shorter amounts of time. One woman who resided in 38106 for 2 years said:

“...I'm not gonna walk the streets, I'm not gonna walk, you know, that's not a good thing because I live in a bad neighborhood, so I wouldn't walk the streets, that's not a healthy thing for a female to do anyway... I live in South Memphis, and it's drug infested, prostitutes walk up and down the street, drug addicts walk up and down the street, I have to keep a reality because I don't see people doing this, but I mean, you know, you know when you see people, you know the crime rate is high in the area, I mean, I don't actually see people using drugs doing 
down the street, but I mean, you can pretty much tell. You know, I just use my judgment on it you know." -Participant 001

Another woman, who reported being comfortable in her neighborhood and therefore was comfortable with an area park, suggested that her children were also familiar with the area crime and were uncomfortable with her walking alone:

"Nope. I used to, we got a park here right down the street, I used to, I can't do it. I can't do it, my boys are not gonna let me do it. I used to get up early in the morning we got like - it's a thing where you can walk around it, they call it the walk park - I can't go up there they won't let me do it...[My sons] say I can't go [to the park] by myself anymore. They say I can't do it. I used to have a walking partner, but my walking partner don't live out here no more. I would feel safe, I would feel alright [walking], but they don't." - 003

Sociocultural aspects of participant lives and their environments were found to be highly significant in encouraging, or discouraging, consistency and health maintenance within this group of women, based on their responses. Additionally, social support was very important in establishing patterns of consistency and accountability for physical activity, thus contributing to weight loss. Without social support, many women defaulted to either going outside of their residential neighborhoods to walk, finding other forms of activity to participate in, or engaging in no activity at all.

\section{Sociocultural factors - The effects of community and social support on health, weight loss, and physical activity}

Social support, in the form of neighbors within residential neighborhoods and family, was indicated as extremely important, as well as the most consistent factor among the study participants, in facilitating long-term physical activity and health maintenance, ultimately leading to weight loss. In most cases where women reported sustained physical activity and positive habit change, there was either a substantial supportive family presence, an indication of a close knit neighborhood and substantial neighborhood social ties, the presence of others while engaging in physical activity, accountability in the form of observing the effects of generational diabetes, which was also substantial among the study participants, or perceived community safety.

Although there were several factors that were intimately related to sustained and continual health maintenance, social support and substantial social ties appeared to be the common thread among women who also reported consistent physical activity within their residential neighborhoods. Dimensions identified relating to positive influences on weight loss, health maintenance, and physical activity included (1) close knit neighbors within the residential neighborhood; (2) neighborhood watch; (3) law enforcement presence; (4) others' presence during engaging in physical activity, and; (5) the perception of living near older neighbors. 
Accountability through children's reminders of what was right and wrong, a desire to remain healthy for grandchildren, discussions of food consumed, sharing healthcare provider conversations amongst family and peers, and even neighbors joining in exercise were reported to help reinforce necessary changes of diet and exercise patterns.

One woman described the frustration that can come with social support and accountability, even though it is helpful:

"My mom, she stays on me all the time, and matter of fact, she gets on my nerves, because I try to tell her, you don't know how I feel you can't tell me how I feel unless you've walked in my shoes before. It's been moments that I just get so frustrated because I have to stick myself every day, I have to [have] 4 shots a day, I check my sugar 4 times a day, you know, my fingertips are sore, I'm ashy, and you know, my chest crawls, I break out, my face breaks out, you know, I've been - I've went through it but my family has been right there, right there." Participant 005

In addition, positive external accountability was mentioned by 8 women as contributing to their weight loss and health maintenance efforts. Family support served as an encouraging factor in engaging in physical activity and weight loss.

With regard to physical activity, established roots and ties to neighbors was the biggest contributor to physical activity engagement within the residential neighborhood. With increases in social ties and length of time in residential neighborhoods, women knew their neighbors and felt safe, regardless of condition of the social environment, where crime was readily noted to be high. Women who expressed this sentiment had no problem walking, sometimes even walking with children in the neighborhood, or walking to other family residences as their form of physical activity. Neighborhood watch, the perception of a substantial law enforcement presence, and older neighbors also contributed to the perception of safety and encouraged physical activity. Two of the participants discuss walking in the context of comfort and having significant social ties:

“...if I walk to my sister's house from my house, it may be about 20 minutes. They say I'm crazy, because I do it all the time but it might take me 15 minutes it depends on how I feel that day, it might take me 30 minutes, sometimes it takes me 15 minutes to get there...yeah, I'm from this neighborhood, well, I [grew up partly in] Midsouth Memphis, I grew up partly over here and partly in Orange Mound, so everybody knows me over here. I mean, everybody. I've been over here for 17 years I done fed children (sic), I done clothed children, I done wiped snot, I done did it all."- Participant 003

An additional participant reports:

"I feel good about walking around the neighborhood because for one thing, I have been driving lately, and a lot of this medicine that they've got me on, it says do 
not operate a machinery or whatever, sometimes I'm dizzy, so it does me good, just to be right here, around my home...we got a good community here, we've got neighborhood watch, all of our neighbors are close knit, all of our neighbors, we look out for each other. I can walk at nighttime it doesn't bother me. You know what, everywhere you go it's something going on anyway, but you know, around here, they don't - people don't really bother you because like I said mostly everybody that's over here has been here for years." - Participant 005

When women reported either being in the neighborhood for a shorter period of time or a lack of substantial social ties within the residential area, a common sentiment was the perception of being unsafe engaging in outdoor physical activity in the neighborhood. The observed relationship between physical activity and social relationships was integral in many women's decision to engage in physical activity. Limited external accountability encompassed both family and outside social relationships. In this context, women would hesitate to walk by themselves and would opt to either walk in other areas, walk with other people, or not walk at all. This was expressed by women in both zip codes. One woman who lived in the 38106 zip code for 3 years stated:

"Well I never really went by myself, I would also go with my fiancé or his mother, so I would never really go - in another favorite spot, matter of fact this is where I went walking the most. It was on the Riverfront in Harbortown, and I felt very safe there. But it was never by myself, we would go together...I prefer a lot of other people around..." - Participant 007

Another woman who lived in 38109 for 7 years acknowledged that she primarily walked in parks due to the lack of sidewalks in the area. However, she expressed being uncomfortable walking in parks by herself:

"Even now when I walk in the park, if there's not that many people in the park or if there's not any women, I won't walk." - Participant 011

Women who were uncomfortable walking by themselves in 38106 typically appreciated a law enforcement presence, which was noted to play a role in encouraging physical activity. This generally was not expressed by women in 38109 because most women indicated lack of sidewalks as the major deterrent for engaging in physical activity, regardless of a law enforcement presence. One woman from 38106 discussed crime in the area and stated that this is why she preferred to use the park, if anything. She states:

"Because they have a security guard in the gym there where the park is, and they - and it's overseen, I mean they can just - cameras are out there I mean, I just feel safer that way in case something does happen or somebody does try to - rape you or rob you or whatever, it'll be on tape, or film, or something." - Participant 001 
Older neighbors contributing to the perception of safety and potentially encouraging physical activity in the residential neighborhood was a minor theme particularly in 38106. Again, although crime was acknowledged and expressed as a deterrent to physical activity, the presence of older neighbors provided comfort and a perceived "shield" from other perceived dangerous parts of the area. One woman said this of the differences expressed as "pockets" within the ZIP code:

"I mean, I don't ever go there to the store, like on the Bellevue and Parkway, it's kind of rough down there, I wouldn't go down that end of my ZIP code, they [are] kind of - guys hang out there and you know. But in this little area over here, it's kind of older people, you know." - Participant 008

In 38109 , older neighbors also seemed to contribute to perceptions of safety; however, lack of sidewalks tended to overrule this and did not necessarily contribute to physical activity, with one respondent from 38109 noting the following:

"This neighborhood is basically kind of a quiet neighborhood, and people have lived over here for years, and so I live like right on a main street, and if we have people walking, it's probably somebody going somewhere else. As far as the people in the neighborhood, everybody basically has cars and you don't see too many people walking over here." - Participant 018

Counter to aspects of the social environment and social support reported to assist in positively facilitating consistent health maintenance, factors related to social environment were noted to also be particularly discouraging and were reported to potentially negatively influence weight loss, health maintenance, and physical activity. Dimensions related to negative social influences included (1) presence of neighborhood crime; (2) generational diabetes influences; (3) perceived lack of family support; (4) inactive neighbors, and; (5) perceived social norms. Social factors was additionally identified as a dimension of many other main influences that positively and negatively affect health maintenance.

Crime was a major socially-related factor that significantly deterred physical activity, and was expressed by residents of both 38106 and 38109 . However, women of 38106 expressed this concern more consistently and predominantly. In this study population, three women indicated significant weight gain since being diagnosed with type $2 \mathrm{DM}$; each of these ladies expressed considerable concern with physical activity associated with crime in the neighborhood. Generally, concern about crime with regard to physical activity was communicated as discomfort in walking alone, particularly when no substantial social ties had been established. The rationale of not walking relative to crime in the neighborhood was aptly expressed by one participant:

“...I'm not gonna walk the streets, I'm not gonna walk, you know, that's not a good thing because I live in a bad neighborhood, so I wouldn't walk the streets, that's not a healthy thing for a female to do anyway...I live in South Memphis, and it's drug infested, prostitutes walk up and down the street, drug addicts walk 
up and down the street, I have to keep a reality because I don't see people doing this, but I mean, you know, you know when you see people, you know the crime rate is high in the area, I mean, I don't actually see people using drugs going down the street, but I mean, you can pretty much tell. You know, I just use my judgment on it you know." - Participant 001

However, even when substantial social ties had been established, an interesting dynamic occurred with one woman, where there was still expressed discomfort in walking alone, and who also lived in the area her entire life. This particular woman would walk in a group, but preferred not to walk on her own, being affected by her perception of crime in the area. However, she also had many social connections who were also diabetic, which may have played a role in her health maintenance and weight loss. Another respondent succinctly summarizes her perception of the experience of area crime and engaging in exercise in comparison to having diabetes:

"It's a gym right down the street, and community center. I wasn't going down there, they said somebody got killed down there, I wasn't going down there and be a victim. I rather diabetes to take me out than a bullet." - Participant 015

Lack of family support for women to maintain their health was reported by several women as severely discouraging in any attempts made to modify health habits. Although nutrition was one dimension consistently indicated by the study participants, another dimension reported included one woman discussing how she felt her husband was unsupportive of her, contributing to a degree of resignation toward potential health changes until she could leave that situation:

“... my oldest son's dad, he's 22 , so it's a long time, but I'm just tired, fed up. So I guess when I get out this situation, I can do better. He's not a person that, you know how some husbands motivate their wives and be there for them? He's not like that. So if you don't have nobody that can motivate you, I mean, keep you [from] feeling bad and down, you don't need that, and I know, I'm 42, so I know I don't need to be going through that so." - Participant 012

Many women reported that their neighbors typically did not engage in physical activity. Health promoting activities were indicated as not a social norm seen in the residents of the area. In fact, women indicated that many typical behaviors seen were generally counterproductive to healthy habits. Women indicated that neighbors ate "junk" food - food high in fat, low in nutrient content; smoked; consumed alcohol regularly; and diabetes and hypertension were standard conditions seen amongst their social relationships. When asked about active neighbors, one woman stated the following as typical of what was seen in terms of her neighbors engaging in exercise:

"If [neighbors] were going to exercise - let me tell you something. If they were going to exercise, this lady used to walk all the time, I used to think she was exercising. Girl she was walking to get those two 40's [beer] from the store and 
walk back home and sit on the porch. And then it dawned on me." - Participant 015

The quality of relationships established in the community, and the degree of social ties contributing to self-accountability was a theme identified as contributing to successful weight loss and health maintenance. This appeared more often in association with women who reported consistency in their nutrition and physical activity regimen. Additionally, the perceived social environment of the women had a significant effect on efforts taken toward establishing consistency in nutrition modifications, physical activity, and maintaining health though medication adherence. However, the effects of crime, generational diabetes, and perceived lack of family support, along with inactive neighbors and neighborhood social norms, served as a deterrent toward successful health maintenance and weight loss among the study participants.

\section{Influences of the Physical Environment that Encourage and Discourage Weight Loss and Health Maintenance}

Environmental influences within the context of this study were characterized by either perceptions regarding conditions and characteristics of the physical environment and its effects on physical activity and health maintenance; reporting by study participants concerning presence and density of resources available in their ZIP codes; and accessibility and walkability of the environment as perceived by study participants. Encouraging factors frequently mentioned were (1) parks and (2) mobile farmer's markets. Several discouraging influences were identified and included (1) inadequate food options; (2) stray animals; (3) lack of diabetes-related information resources; and (4) lack of sidewalks.

\section{Encouraging environmental influences - Parks}

The availability of nearby parks were perceived to allow women in both zip codes to engage in physical activity and were reported as the most encouraging aspect of the built environment for physical activity. Although many of the study participants chose not to utilize the parks, indicating them as high crime areas, other women suggested that they did not mind walking in parks. One woman states the following about utilization of parks in the 38106 area:

"Yeah, I'm okay with walking because they have a park up here, I go up there, me and the kids and I walk while they're playing I'll walk around the park, yeah. Something to keep me motivated or something, where I take the kids to the park and stuff. I normally walk three times a day, like in the morning, [at] noon, and then 3-4 o'clock when the kids get out of school, I walk and pick the kids up, and we'll walk all the way around and come back." -Participant 004 
In the preceding quote, participant 004 also indicated that she knew many people in the neighborhood, many people knew her, and she was from the neighborhood, so she had no problem walking in the parks despite crime perceptions. Women who indicated that they utilized parks physical activity also indicated connectedness socially, or indicated that the presence of security cameras contributed to comfort in walking in parks.

\section{Encouraging environmental influences - Mobile markets}

Mobile farmers markets, and access to the local farmer's market in the area, provided women in 38106 with varying options and access to fresh fruits and vegetables of higher quality than those found in the area grocery store. Many women reported that they liked this aspect of their neighborhood. One woman stated:

"Well I have to - there's a market man that rides through, he has fruits and vegetables on the truck when he comes through I always buy some from him, keep them in my house for me, so I can have fruit and stuff, the stuff that I need." - Participant 004

In contrast to encouraging influences, there were significantly more discouraging influences reported. One discouraging influence mentioned was the quality of produce in the local grocery store. Many women perceived that the local area grocery store not only carried poor quality produce - they also perceived that because it was located within a minority neighborhood, there was an expectation that this was inevitably so. Most participants from the 38106 area stated that it was necessary for them to leave their residential environments, and travel to other grocery stores farther away that sell better quality produce. One woman describes her perception of the area store:

"We don't have but one grocery store, that's called Save-a-Lot. No ma'am, we in a black neighborhood, so that means what? We don't have good produce over here. So that's why I have to go out the neighborhood, I have to go out the neighborhood to buy my vegetables and different stuff like that. You know, basically my vegetables and fruits, I have to go out the neighborhood to get fresh fruit and stuff." - Participant 003

\section{Discouraging environmental influences - Stray animals}

An additionally frequently mentioned characteristic that was a deterrent to physical activity was the presence of stray animals. Several women indicated that this was a significant problem in both ZIP codes. Some women reported not necessarily being frightened enough to discontinue walking; however, they still acknowledged this as a potential threat to their safety and perceived that this may affect others in their decisions to engage in outdoor activity. Other women suggested that they absolutely do 
not walk because of their fear of a dog attack. One woman said the following regarding her view of stray animals how others may feel as well:

"They should work on fixing all these stray dogs be running around, because you when you're out there walking, and you're walking by yourself, dogs don't care, they will attack you. But I always have a stick when I'm walking so, run them off, yeah. They need to get rid of the dogs and cats that run around here, the stray dogs and cats. And some people's dogs and cats be in their yard but the fence, instead of them keeping their fence locked with the dog in it, they'll have it wide open and the dog will just run out and bite people, and I be trying to tell them y'all need to watch the animals y'all got in the yard because it's more than me walking around here. Because I'm not scared of them doesn't mean anyone else won't be scared of them." - Participant 004

\section{Discouraging environmental influences - Lack of diabetes resources}

A lack of diabetes resources in the 38106 area was mentioned by three respondents. This perception was noted to be a missed opportunity to address much of the misinformation that plague many of the residents of the area. One woman stated the following:

"I just wish we had somewhere over here that's closer that people could go to, you know when they got diabetes and stuff, to talk to somebody, that's what a lot of people say... because you know they closed the clinic down here that we had, they closed the clinic that had been here for years, they closed that clinic up, the only closest clinic... and for us to have so many churches that we got, they don't offer nothing. You know you would hear some churches say they finna do a health fair, or have somebody down there to talk to you or something, none of these churches don't do this over here, but they want you to support them, come to they church, I can't come to your church." -Participant 003

\section{Discouraging environmental influences - Lack of sidewalks in 38109}

Limited availability of sidewalks for use during physical activity was a common sentiment among women who reside in 38109. Most women in this ZIP code suggested that lack of sidewalks discouraged efforts to engage in physical activity. One woman stated:

"We don't have sidewalks. The street is unsafe, and there are no sidewalks, so that in itself is a danger. We have several neighbors including myself, calling and complaining about [the lack of] sidewalks. And there is a park not far away but... if you don't have transportation how would you get there. You have to walk, and it's a very dangerous walk." -Participant 013 
Many environmental circumstances were reported by women; most of these were perceived to be discouraging in nature. Regarding the scope of influence of sociocultural, environmental, and economic factors that discourage physical activity and health maintenance, environmental circumstances were noted to be secondary to sociocultural influences, and particularly, social influences on weight loss, physical activity, and health maintenance.

\section{Economic Factors that Affect Health Maintenance}

Economic and financial considerations were identified as primarily discouraging consistent health maintenance and weight loss. Dimensions identified as economic considerations included (1) cost of medications and medication equipment; (2) lack of health insurance and perception of poor quality providers; (3) limited financial resources to obtain recommended diet; (4) limits in residential options.

Several women interviewed noted that medications, and particularly insulin pumps, were expensive. Two of the women indicated that because they could not afford the medication, they did not take it although they needed it, and this contributed to poor maintenance of the type $2 \mathrm{DM}$ condition. One woman summarized how having diabetes affected her from an economic perspective:

"It plays a part because it's expensive to eat right, and I don't really have a lot of money. Everything has went so expensive and you're trying to work, pay bills, eat right, and buy medication and it's expensive sometimes." -Participant 019

Additionally, many women indicate that they travel outside their proximal environments to obtain better quality foods. Another participant states the following:

"Yeah. I know they [inaudible] probably don't have the transportation and then too, that might not have the money. I don't have the money but I, you know, I get a little food stamps but I still rather go to Kroger or somewhere, I might have to spend a little more but you know, to get the stuff that I know I kind of need for my health." -Participant 008

Two women indicated that having health insurance was associated with positive health maintenance. The very act of possessing health insurance encouraged these particular women to take better control of their health. Once there was no insurance, there was decreased motivation to obtain the healthcare needed to maintain their health, thus discouraging any weight loss attempts. One woman indicated that when she previously lost weight via Weight Watchers, in which participation was funded through her health insurance, she lost significant weight; after her insurance coverage lapsed, she no longer had the resources to continue attending the program:

“...I was losing [weight] because I was eating right and I was doing what the program had to do - I was losing good, but after you stop going, because when I 
was going I was going through my insurance, so when my insurance expired it expired and I never got a chance to get right back up..." - Participant 010

Another woman indicated that she perceived acquiring better health insurance to extend her care options, which she subsequently believed provided access to higher quality providers:

"And then people don't understand they outgrow their doctor. That doctor has seen you for years, has done all that he can do for you, and it's time for you to go to somebody else. So now, at the time, I was going to [name withheld] I really liked him, he do listen, but he was at Christ Community, and at that time, I didn't have any insurance, that's why I was there. But now I have another job and we have insurance so I go to [name withheld] and he listens, and he has a nurse practitioner and she listens. And I love both of them. And I am doing fine." Participant 020

Economically, some women's perception of health care quality received was tied to them having health insurance. With the perception of seeing a poorer quality health care provider, particularly when women did not have insurance, came the perception of poorer health, and considerable frustration was observed to follow in some cases. One woman states:

“...if I had some insurance I would personally get a doctor that I can do some one-on-one with, because the Med, they just give you this medicine and tell you to go ahead on, you have to read up on it and their education class, they have you there so long, like 4 or 5 hours...I pray that God would fix it to give me some type of insurance, you know, that I can go to the better doctor. I'm not saying the Med is not good, because they're gonna take you whether you have insurance or not, but I just feel like if I had some insurance I could get more of one-on-one time, that I need, versus not having any." - Participant 005

Women additionally reported that limited financial resources limits choices in terms of residential neighborhood chosen; women perceived that their limited incomes had an effect on where they could live, and with limited financial means came the perception of limited options in terms of residential environment. Limited options appeared to affect psychological considerations, which could also contribute to the depression and resignation seen among many of the study population. One woman stated the following:

“... I'm here for now, for financial reasons, but I do - I'm looking forward to moving...so I'm staying until [I reach] my [financial] goal." - Participant 013

Overall, economic factors appeared to play a significant role in the perception of how well women felt they could take care of their health and lose weight, given their perceived circumstances. With circumstances reported including limited access to adequate foods, perceived poorer health care quality and lack of insurance, many women 
conveyed that financial circumstances may negatively impact the ability to lose weight and adequately maintain their health. Limited resources introduce another perspective on how women manage their diabetes. One woman indicated that she could not afford the insulin, so she had to manage it through diet and exercise. Another woman indicated that financially she had to shop for groceries on sale and was limited by area resources. In 38106, several women indicated that Save-a-Lot was not a good store, but it was one of the only resources in the area.

\section{Part 3: An Analysis of Environmental and Structural Barriers in Achieving Weight Loss Using Geographic Information Systems - ZIP codes 38106 and 38109}

Part 3 of the analysis addresses research question (3):

3. What are objective environmental and structural barriers present that can affect weight loss and health maintenance in 38106 and 38109 ZIP codes, which can gauged through Geographic Information Systems?

Geographic information systems (GIS) was used to gauge objective environmental barriers that may be present in the 38109 and 38106 areas. The general scope of this analysis encompasses the entire 38106 and 38109 ZIP codes, includes residential populations, and depicts aggregated accessibility to resources based on population saturation in various areas of each ZIP code. Resources of interest depicted in this analysis included (1) community centers, (2) convenience stores, (3) farmers markets, (4) grocery stores, (5) health facilities, (6) parks, and (7) transit stops. Each resource type was chosen based on its potential contribution to weight loss, health maintenance, and physical activity among the study population. Barriers to weight loss and physical activity, for the purposes of this analysis, were classified as low percentages of area walkability to resources based on walking time and proximity, and an assessment of the quality of the listed resources as reported by participants and other accounts through field exploration. Community centers, convenience stores, grocery stores, farmer's markets, and parks were chosen for field exploration. Health facilities cannot be adequately assessed for initial quality through field observation; transit stop locations were generally found to be uniform and standard in quality, with no discernable features precluding use.

\section{Community centers $-\mathbf{3 8 1 0 6}$}

There were three community centers identified in 38106. The degree of walkability within the 38106 ZIP code to the identified community centers is depicted in Figure 4-2. Based on a 10 minute walking distance, it was estimated that approximately $13 \%$ of the population of 38106 are within the specified criteria. Quality of each community center was assessed via conversations with community center directors and formal visits to each facility. The following field note gives insight into internal workings from the director and assistant director from Pine Hill Community Center, and also characterizes the sentiments from the other community centers: 


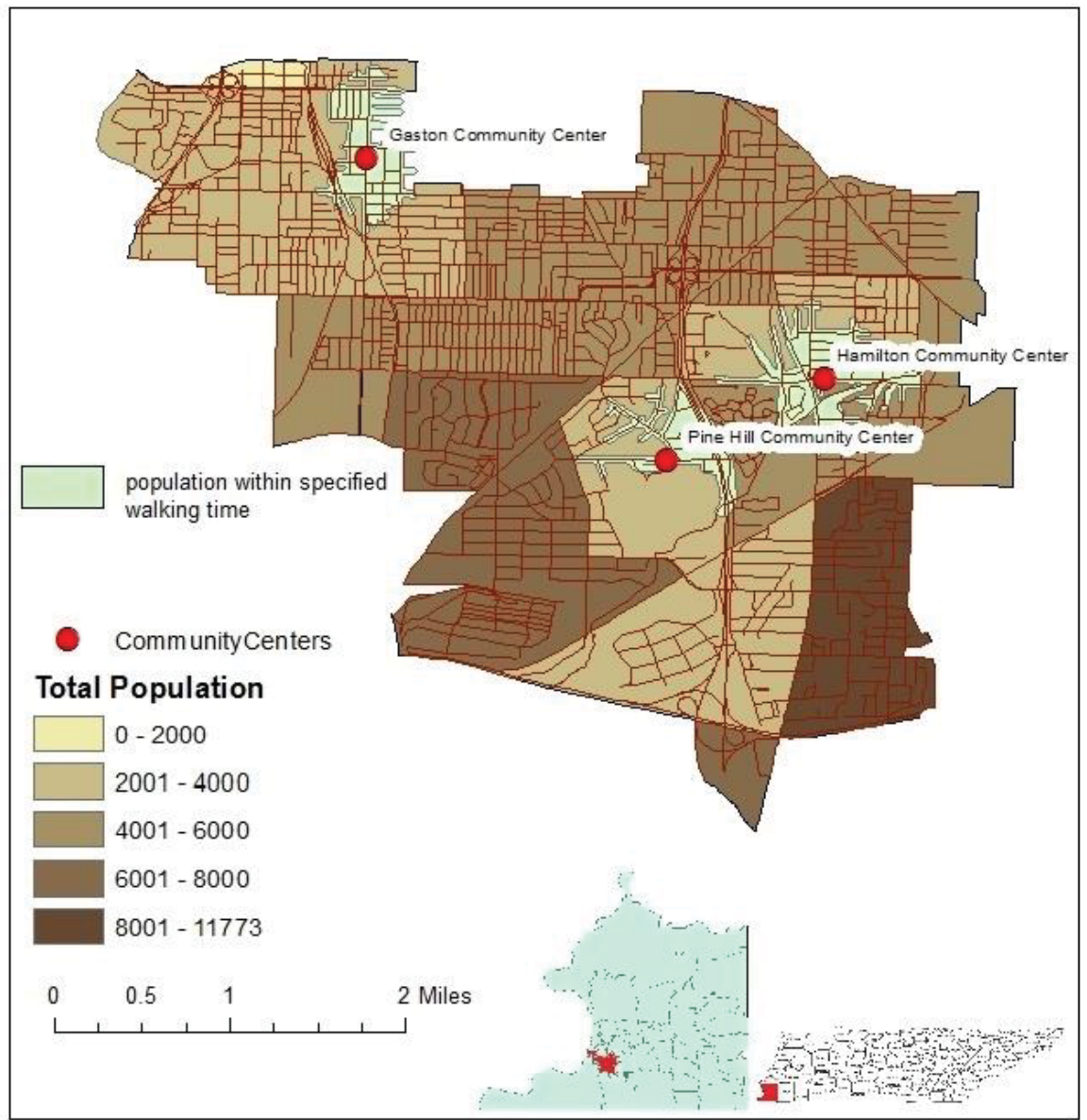

Figure 4-2. Residents in 38106 within 10 minute walking distance to community centers. 
Field note \#3: 3/22/2014 - Location: Pine Hill Community Center

Walking into the community center, it is apparent that the basketball court is the center of activity. In stark contrast to the community center in 38109; there's limited activity. The community center assistant director and director were happy to speak with the researcher. The director indicates that although they desire additional programming within their facility, the City of Memphis keeps strict tabs on all programs held, particularly from a financial standpoint. As a result, they hesitate to host programs because they feel it [the community center] is treated unfairly. The primary concern seems to be financial in nature; they indicate that the City of Memphis takes money that they raise and does not allocate enough funding to their specific facility. They also indicate that most residents hesitate to visit because "gangbangers" frequently play basketball on the premises. They also mention that even basketball tournaments have become violent, which caused cessation of the youth league. They note that there are no consistent programs at the center dedicated to health, although they do have summer programs. They have a knitting group comprised of elderly women that use the facility 3 days a week in the morning, and they also have a volunteer group that serves hot meals every weekday at $5 \mathrm{pm}$. At one point, the City of Memphis threatened to shut down the knitting group, because they expected them to pay to gather. The women rallied and protested, after which the City decided they could host the group there at no charge. The directors also remark that if there were any consistent programs dedicated to resident health, they were skeptical that the residents would be interested. Fitness equipment present is broken and outdated; they indicate that no one ever uses it.

The City of Memphis indicates on the organization website that the mission of community centers is "to provide a safe and honest environment that will offer a wide variety of programs and services which meet the identified needs of the community." They also note that community centers should be "a safe place for all ages to meet new friends, socialize, learn new skills, keep fit, and stay healthy." However, it does appear that not all of the community centers in 38106 meet that description on a consistent basis.

\section{Convenience stores $-\mathbf{3 8 1 0 6}$}

Forty-two convenience stores were identified as available within the 38106 area. The degree of walkability within the 38106 ZIP code to the convenience stores is depicted in Figure 4-3.

Based on a 5 minute walking distance, it was estimated that approximately $27 \%$ of the population of 38106 are within the specified distance criteria. Quality of a sample of convenience stores was assessed via conversations with participants and formal visits to representative locations, with observations of the primary goods offered within settings visited. Insight into representative locations is summarized in the account below: 


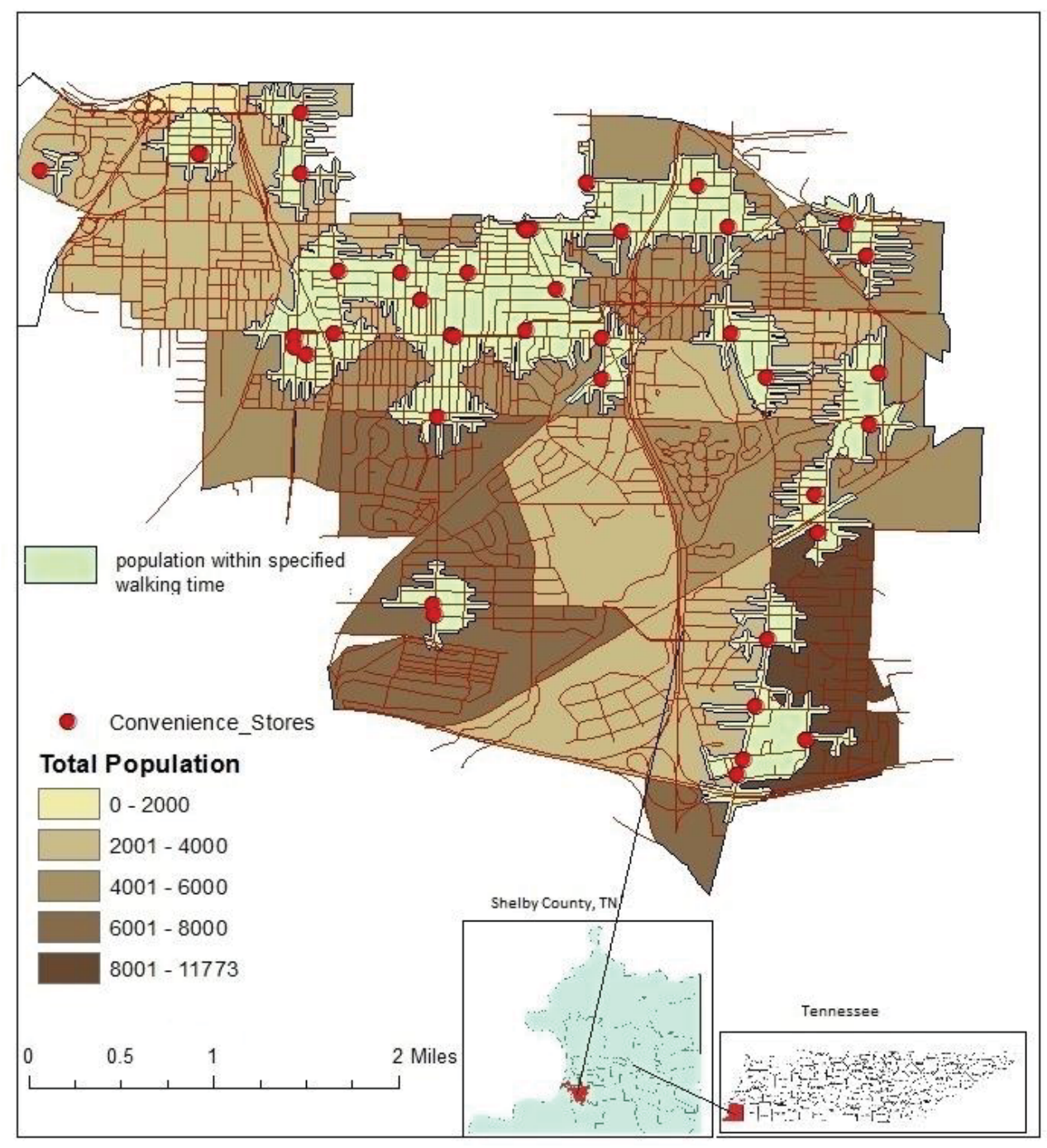

Figure 4-3. Residents within 38106 within 5 minute walking distance to convenience store locations. 
Field note \#5: 3/25/2014 - Location: South Parkway Food Market

Inside the convenience store, it appears to be similar to other filling stations

outside the area. Researcher asks clerk at the cash register if they sell any fresh fruits, vegetables, or produce. The clerk replies that no fresh fruits, vegetables, or produce are available. A large selection of beer and spirits is noted. Other typical offerings are also present: chips, candy, soda, cookies, etc. Clerk also indicates that convenience stores comprise a large selection of available food in the immediate area, as there are not many grocery stores or restaurants.

\section{Grocery store - 38106}

Only one grocery store was identified as available within the 38106 area, corresponding with participant accounts of the area. The degree of walkability within the 38106 ZIP code to this grocery store is depicted in Figure 4-4. Based on a 10 minute walking distance, it was estimated that approximately $5 \%$ of the population of 38106 have access to the grocery store via walking transit. Quality of this store was triangulated from participant responses via a formal visit to the location, and an observational exploration of the grocery store and goods offered. The following field note depicts the field exploration:

Field note \#2: 3/5/2014 (grocery canvas \#1)- Location: Save-a-Lot, E Parkway, Memphis, TN 38106 Immediately upon entering, the turnstile located at the entrance is surprising. It's noted that this presents a danger to the people in the store if there were an emergency, as this is the most obvious exit besides the checkout lanes. The first section visited was the produce offering. Researcher notes that the oranges are brown and old. Onions have full grown plants growing from them, indicating that they had been there for an indeterminate time. There are fruit in syrup containers available. A closer inspection of the produce shows that with the exception of turnip greens, all the produce appears to be of low quality. No organic produce is available. Walking toward the meat offerings, large quantities of pickled pork are available - pickled pigs feet and various sections of pig appear in jars in a freezer. No organic meat appears to be available. Small section of chicken breasts in contrast to the large selection of other cuts of chicken. Moving on, freezer appears to be the same as other grocer freezers. It is noted that there are cakes and pies adjunct to every aisle. The offering of cakes, pies, and cupcakes is larger than the offering of produce. An alcohol display featuring various types of beer appears just before reaching the counter checkout.

Field note \#21 (grocery store canvas \#2): 8/9/2014 - Location: Save-a-Lot E Parkway, Memphis, TN 38106

In this second canvas, there is again a turnstile located at the entrance. The point of the turnstile, according to the manager, is to prevent theft and to better keep track of people in the store and what items they leave the store with. The store manager was also asked whether people walk to the store - he replied that "people 


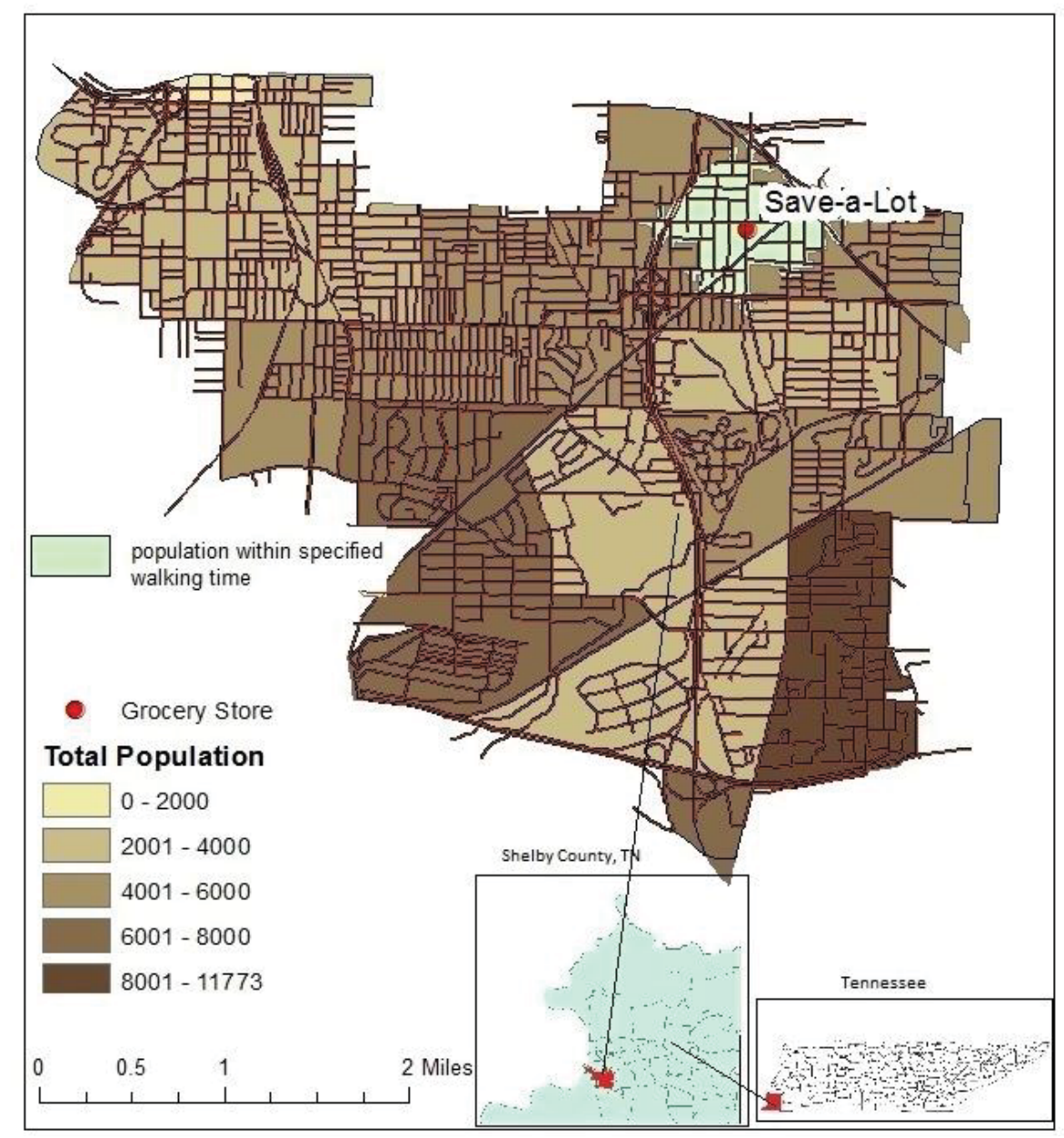

Figure 4-4. Residents of $\mathbf{3 8 1 0 6}$ within 10 minute walking distance to area grocery store. 
may walk [to get here], but a lot of the time you'll see drunks walking here to get beer". Immediately beyond the turnstile (which is the only entrance point within the store) the first items for sale are 7up cake, chocolate cake, beef ravioli, and various condiments (mustard, sugar, barbeque sauce, ketchup, etc.). There are also selections of snack foods typically found in convenience stores (potato skins, potato chips, etc.) before coming to the produce section. The produce selection in this grocery store location consisted of the following items: potatoes, sweet potatoes, yellow onions, tomatoes, apples (green, golden, and red). Other items included True Fruit brand fruit cups, peaches, strawberries, watermelon, iceberg lettuce, green bell pepper, red bell pepper, cucumber, squash, cabbage, and various types of greens (mustard, turnip, and collard). An additional observation is that the quality of the produce is low. A store clerk indicates that he perceives that the food gets old because there is a low demand for the produce. Also available were several varieties of preserved and processed meats (for example, preserved country ham, several varieties of hot dog and lunch meats). This is notable because the selection for preserved meat is larger than that of the produce selection, with more space allocated to this section. The leanest ground beef available was $73 \%$ lean, $27 \%$ fat content. Additional differences observed based on the researcher's typical experiences in area grocery stores was the availability of chicken paws, jowl bacon, pig's feet, and pig tails, perhaps reflecting the area's nutrition culture. Also for sale were rib eye, beef liver, chicken wings and chicken legs, turkey neck bones, pork roast, and various cuts of beef. In the dairy section, no unprocessed butters were available - country spread was available for purchase. There were selections of skim (fat free) milk, 2\%, and Vitamin D (whole) varieties of milk. Beer was available for sale in the area next to the eggs and butter. Whole wheat bread was available for purchase. In terms of accessibility, it is important to note that this store appears to be very close to some area residences.

To better observe and parse out potential differences in the selection of items for purchase within the grocery store based on demographics and area demand, another Save-a-Lot location was visited in the Memphis, TN area. This second store was chosen not only to potentially compare across locations based on area demographic composition, but to retain consistency with respect to store brand. According the US Census, the store location canvassed within the 38122 ZIP code is more racially diverse, with area demographics encompassing a population that is approximately $60 \%$ White, $25 \%$ Black, and $22 \%$ Hispanic.

Field note \#22 8/9/2014 - Location: Save-a-Lot, Memphis, TN 38122

First impression walking into the store was that this location had a substantially larger produce selection. There was also no turnstile located at the entrance of the store. The first items that greeted the store entrants was a large selection of fruit, including banana and plums on sale. The produce selection in this location was as follows: potatoes, sweet potatoes, onions (red, white, and yellow), watermelon, banana, tomato, plums, peaches, apples (green, golden, red), oranges, pears, limes, candy apples, strawberries, grapes, spinach, lettuce, carrots, cucumber, red 
bell pepper, green bell pepper, mushrooms, cabbage, celery, cauliflower, radishes, greens (all varieties, including collard, turnip, and mustard), zucchini, cilantro, corn, and squash. The meat selection at this location compared to the location in 38106 was also substantially different. There was smaller selection of preserved meats. Ground beef in $73 / 27$ and $80 / 20$ fat content varieties were also available for purchase. There was also ground turkey present in the 90/10 fat content variety, which was not present in 38106 . Chicken claws, pig's feet, pig tails, and pork jowl were also not available. In the dairy section, almond milk was present. Beer was not as conspicuously present in the store, although it was available. In this location, there was also a large selection of pastries and cakes, although not as large as in 38106 .

The preceding field note demonstrates a difference in the quantity and quality of items for sale (especially considering fresh produce) when compared to the 38106 Savea-Lot location. Walkability is an important consideration; however, although access may be limited by those with no transportation, many of the study respondents indicated that the store in the 38106 is of low quality and insufficient to meet their dietary requirements with respect to diabetes. A comparison of the 38106 store location with a similar store brand in a different area featuring a more mixed demographic reveals overall higher quality and more options available to the customers of the 38122 area when compared to customers of the 38106 area.

\section{Farmer's market - 38106}

Although participants indicated that there were mobile farmers markets available within 38106, no discernable addresses were obtained from either a yellow pages, NAICS, or general internet search, nor were mobile farmers markets identified from known mobile vendors (i.e. The Green Machine) that service the analysis areas. One farmers market was identified as available and possessing an address within the 38106

area. The degree of walkability within the 38106 ZIP code to the farmer's market is depicted in Figure 4-5.

The farmer's market of 38106 was established by the non-profit organization The Works in affiliation with the Centers for Disease Control. Based on a 10 minute walking distance, it was estimated that approximately $5 \%$ of the population of 38106 are within the specified distance criteria.

Field note \#7: 8/22/2014 - Location: South Memphis Farmer's Market, 38106 This building is a small grocery store on the corner of Mississippi Blvd and South Parkway. There is a kitchen connected to the grocery store, where cooking classes are held for the community. Inside the farmer's market, various vegetables, fruits, and lean meats are sold, including varieties not available in the area grocery store. Items for sale include the following: kale, radishes, greens (mustard and turnip) beets, tomatoes, eggplant, squash, cantaloupe, honeydew melons, sweet potatoes, and carrots. 


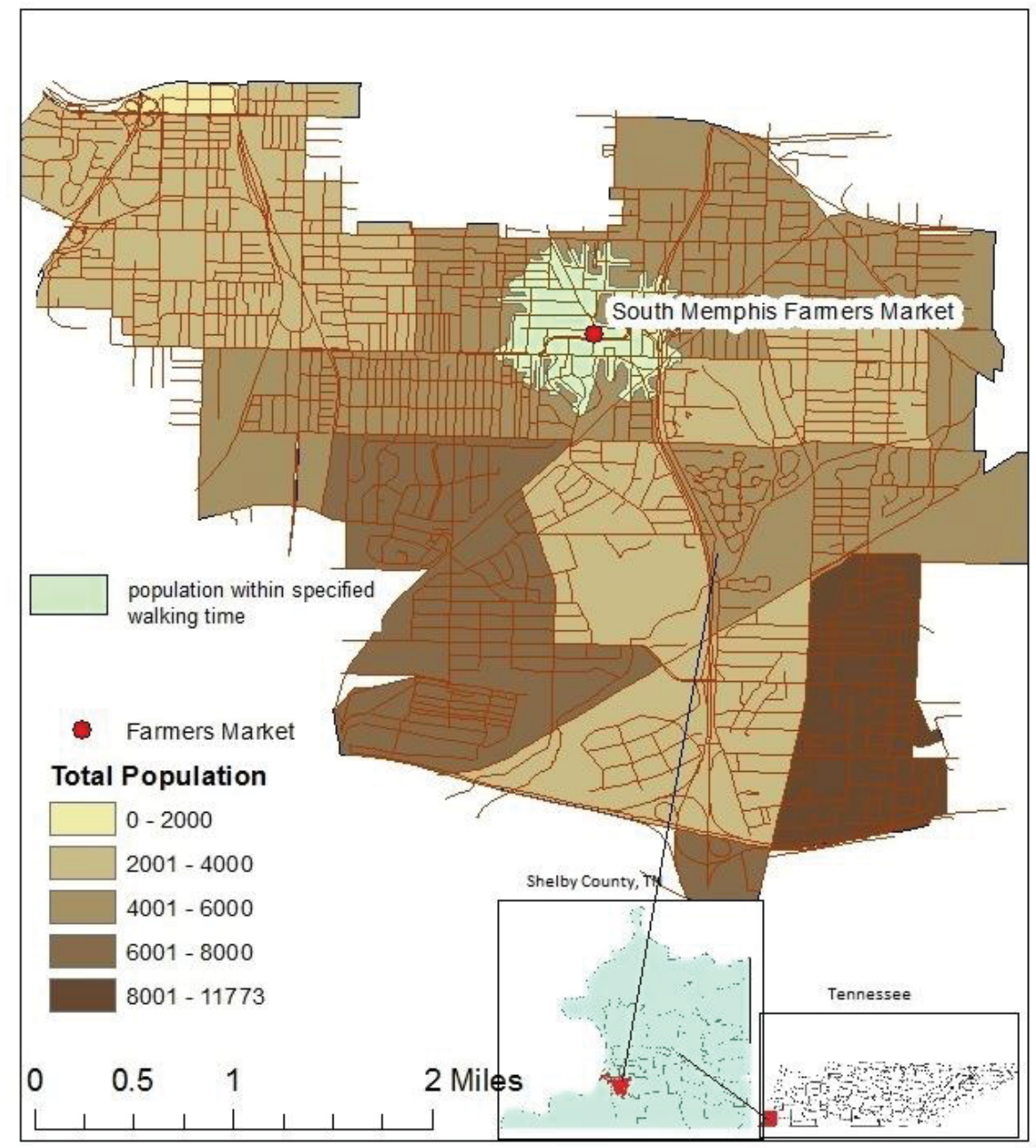

Figure 4-5. Residents in 38106 within 10 minute walking distance of the area farmers market. 
Ground turkey is also sold here, along with leaner varieties of ground beef $(85 \%$ lean, $15 \%$ fat content), and varieties of processed meats, and pig's feet. There is a dry goods selection where they sell items including rice, beans, canned fruits and vegetables, as well as a small freezer selection where they sell packaged frozen goods. The store manager also indicates that a larger selection of fresh produce is sold from April - November every Thursday from 9-6pm. Local farmers and gardeners are invited to sell their quality produce at this time and the fruit and vegetable offerings vary depending on what is available for purchase by local farmers and gardeners. The manager indicates that the most common items available for purchase are tomatoes, cucumbers, and various forms of greens. He also indicates that many people walk to the farmer's market from their homes, and also stop by during these times to purchase items, but he believes many people in the community still do not know about them. The store indicates that they accept Electronic Benefit Transfer (EBT), a system in which state welfare departments distribute welfare benefits onto a magnetically encoded payment card.

Additionally, an area church, St. Andrew's, donated property for the use of the South Memphis Farmer's Market; and awarded a \$250,000 grant to The Works, Inc. to provide a permanent site for the South Memphis Farmers Market to house a commercial demonstration kitchen and a small fresh produce "grocery store", and restrooms.

\section{Health facilities -38106}

There were ten health facilities identified as available and possessing an address within the 38106 area. These facilities were Lemoyne Owen Health Center; Whitehaven Clinic; Walgreens; St. Andrews Community Life Center; Dental Clinic; Randolph Women's Center; Eye and Dental Clinic; Lott Family Dentistry; Omega Rehab Group; and Doctor's Clinic. The degree of walkability within the 38106 ZIP code to the health facilities is depicted in Figure 4-6. Based on a 15 minute walking distance, it was estimated that approximately $48 \%$ of the population of 38106 are within the specified distance criteria.

\section{Parks - 38106}

Seven parks were identified as available within the 38106 area. The degree of walkability within the 38106 ZIP code to the convenience stores is depicted in Figure 4-7. Based on a 10 minute walking distance, it was estimated that approximately $22 \%$ of the population of 38106 have walkable access to area parks. Five parks were documented for field observation.

Field note \#4: 3/22/2014 - Location: Southside Park, Memphis, TN 38106 This small park appears to have a walking trail and a basketball court, upon which there are several people playing a game. The park appears harmless; however; people in the area have indicated that this is one of the more dangerous parks 


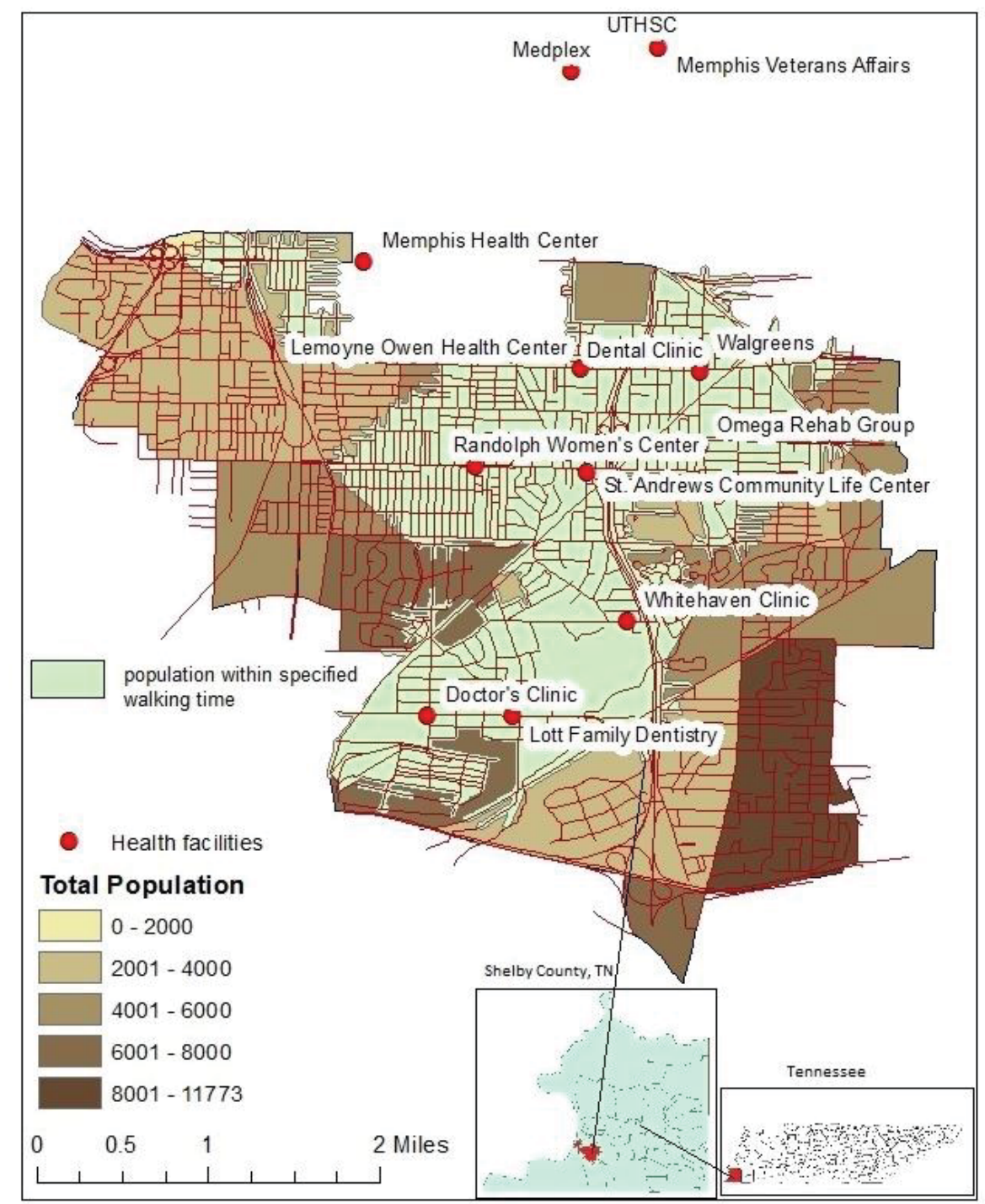

Figure 4-6. Residents of 38106 within 15 minute walking distance from health facilities. 


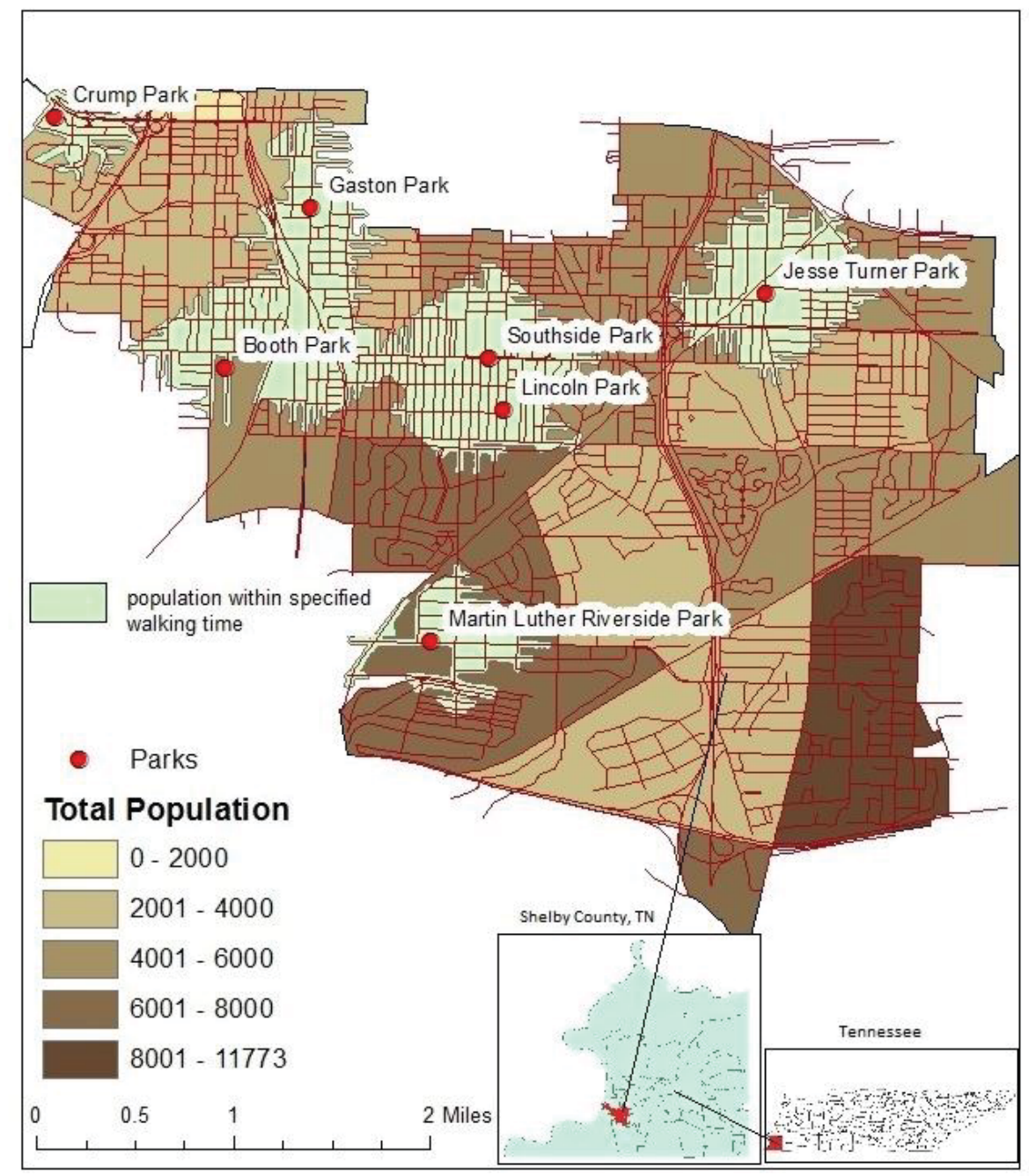

Figure 4-7. Residents of $\mathbf{3 8 1 0 6}$ within 10 minute walking distance of area parks. 
because of the people who frequent. On the outside looking in, it appears to be an ideal place to exercise around the walking trail if one desired to.

Field note \#23 8/9/2014 - Location: Southside Park, Memphis, TN 38106 At this location on this day, there was a police standoff. Yellow tape was present indicating a crime scene around the perimeter of the park and it was closed off to visitors.

Field note \#17: 8/9/2014 - Location: Jesse Turner Park, Memphis, TN 38106 This park is located in a busy area of 38106 . There are residential areas nearby, and the park is accessible through walking with the presence of sidewalks and a nearby transit stop. There is a large baseball field available, with seating for a medium to large crowd. Nearby, there is also a basketball court, a small pavilion, and a playground. There are 3 people walking on the walking trail; one woman is walking with an umbrella because of the high temperature on this day. There is a tennis center located on the grounds. The desk attendant indicates that there are several programs at this center dedicated to tennis and youth development, but that these program are not free of charge. Five large tennis courts are also present on the grounds.

Field note \#18: 8/9/2014 - Location: Lincoln School Park, Memphis, TN 38106 Lincoln School Park is located in a residential neighborhood and is next door to a school. This park may primarily be present for the children in the area - there is a playground and park benches, with a short circular walking trail, and a water fountain. The park appears empty, and there are sidewalks leading up to the park, indicated accessibility through walking.

Field note \#19: 8/9/2014 - Location: Gaston Park, Memphis, TN 38106 Immediately upon arrival, the researcher was greeted with panhandling. Several men who appear to be drinking beer are seated at the park benches under a pavilion. There is a walking trail available, a basketball court, and a softball cage. Generally speaking, the initial impression is that this may not be a safe place to walk for a woman. Several abandoned houses are also noted to be nearby.

Field note \#20: 8/9/2014 - Location: Booth Park, Memphis, TN 38106 The sole feature of this park is a basketball court. On the other side of the street, parallel to the park, there is an overgrown lot with substantial blight and litter present. There are no houses or residences available. In close proximity to this location is a shipyard with crates. There is no walking trail, and the sidewalks are also unkempt and overgrown. The initial reaction to this park is that it is not necessarily a safe place for a woman to choose to walk alone. 


\section{Transit stop locations -38106}

There were 316 transit stop locations, obtained from the Memphis Area Transit Authority, that were identified within the area of 38106 . Based on a 5 minute walking distance, it was estimated that approximately $81 \%$ of the population of 38106 are within 5 minute walking distance from an area transit stop. The degree of walkability within the 38106 ZIP code to each transit stop is depicted in Figure 4-8.

\section{Community centers -38109}

Two community center locations were identified within the area of 38109 . The degree of walkability within the 38109 ZIP code to the community center is depicted in Figure 4-9. Based on a 10 minute walking distance, it was estimated that approximately $5 \%$ of the population of 38109 are within the specified distance criteria. Quality of community centers was assessed through conversations with a community center representative, and formal visits to locations. Field observations are below:

Field note \#1: 8/22/2013 - Location: Westwood Community Center, Memphis, TN 38109

First observation while walking into this location is that there is lots of activity. A representative of the center speaks with me - she tells me that there are several programs held at this location, including a summer youth program, swimming classes, arts and crafts classes, and a youth basketball league. Outside, the pool is brimming with children. Behind the community center is a large park with a walking trail, a basketball court, and benches. It's apparent that if one wanted to walk and lived close, this could be an ideal location. The community center representative indicates that people frequently walk on the trail.

Field note \#25: 8/9/2014 - Location: Riverview Community Center Walking into the community center, there are many children within the center. Speaking with the representative, she indicates that there are many youth programs available, including gymnastics, cheerleading, a computer lab, dance, tutors for kids, and arts and crafts programs. She said many of the programs were aimed at youth, especially during the summer. When asked were there any dedicated programs toward health or health education for adults, the representative responded that there were but they occurred less often. She also stated that they were open to programming pending coordination through the City of Memphis. When asked were there any dangers associated with the community center, the representative responded that there were not; many parents feel good leaving their children to participate in the summer programs and rarely have complaints. This center features a pool, a basketball court, a TV room, and a kitchen that hosts youth cooking classes. 


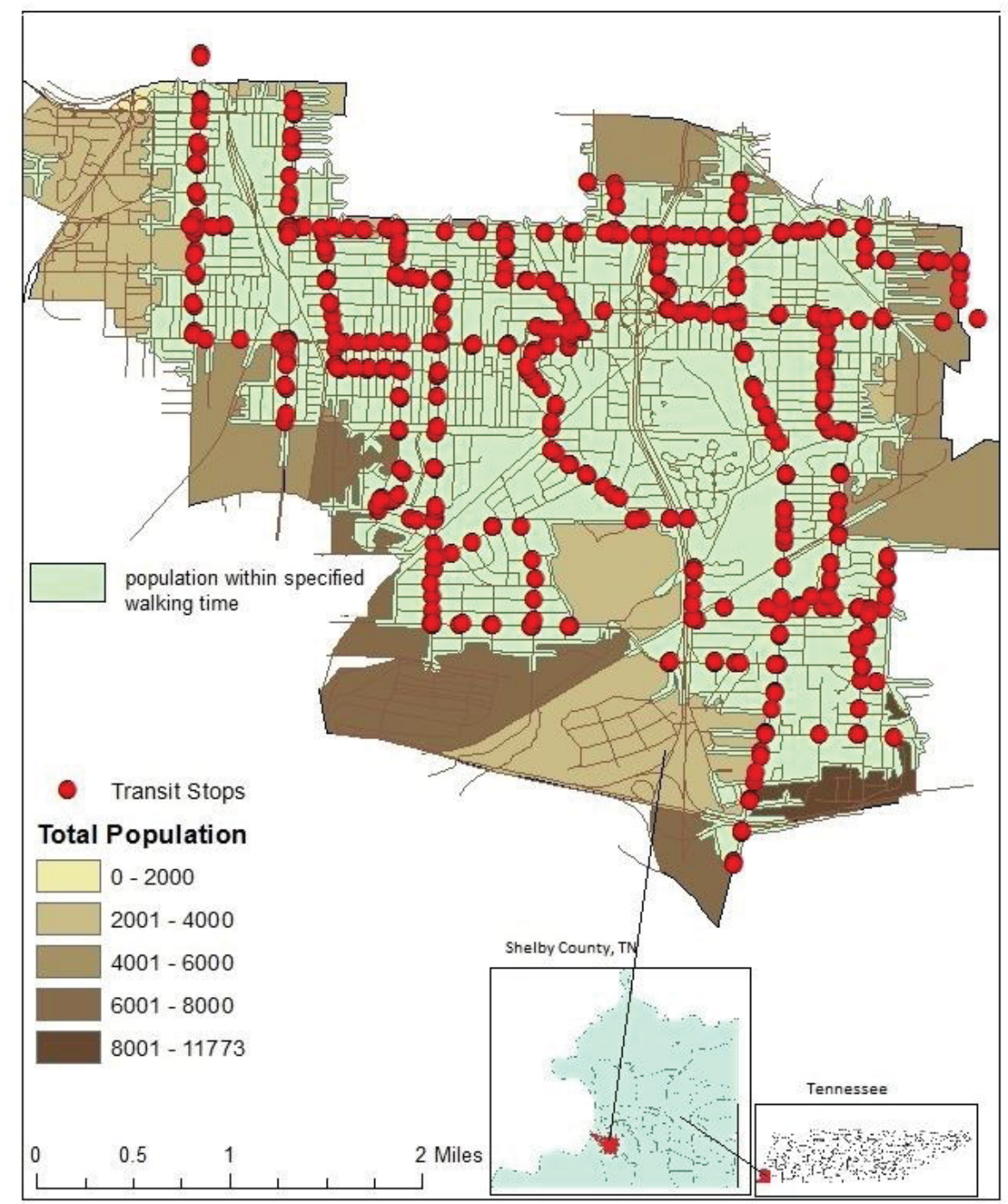

Figure 4-8. Residents of 38106 within a 5 minute walking distance from area transit stops. 


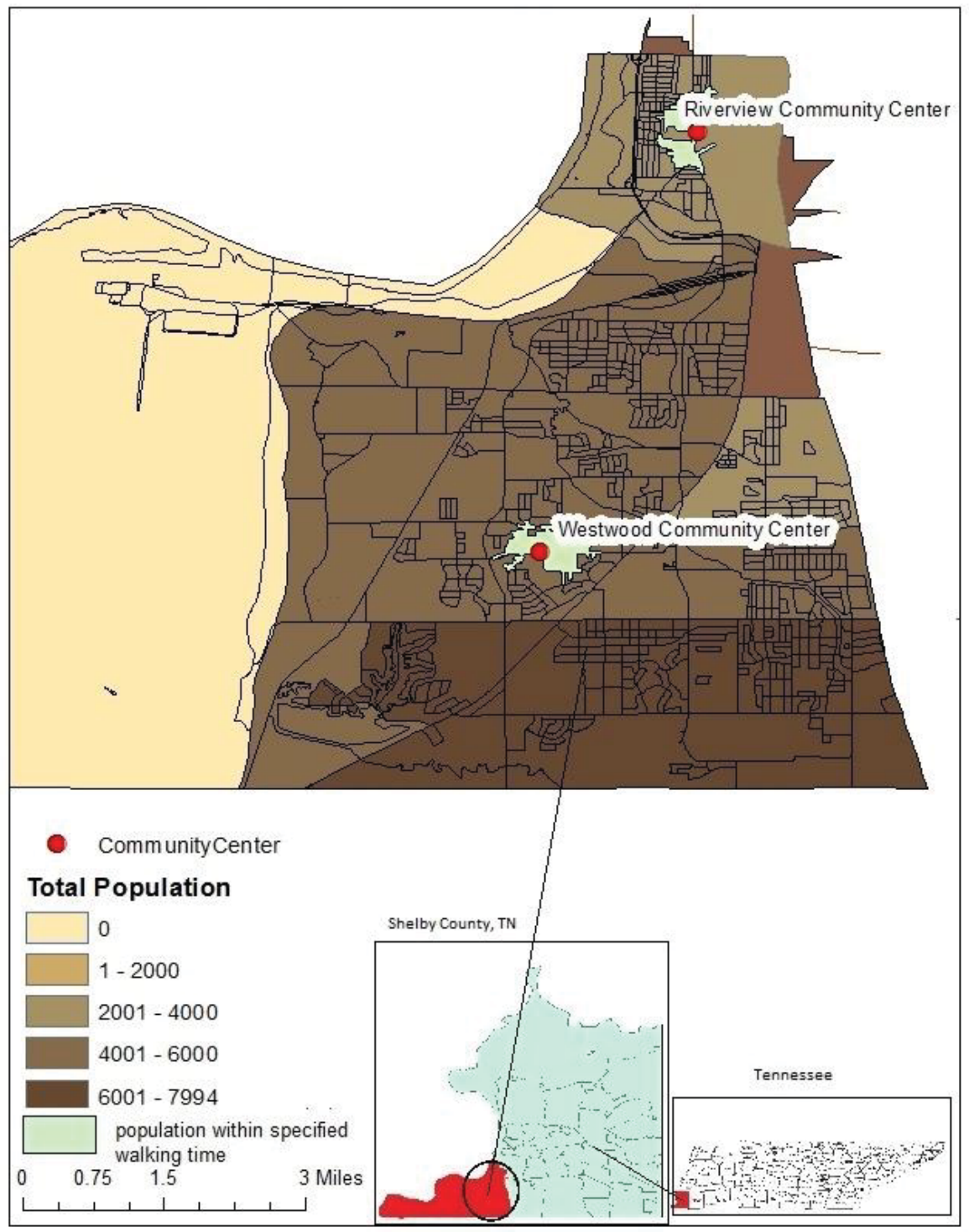

Figure 4-9. Residents of $\mathbf{3 8 1 0 9}$ within $\mathbf{1 0}$ minute walking distance from area community centers. 


\section{Convenience stores $-\mathbf{3 8 1 0 9}$}

Several convenience were identified within the area of 38109. The degree of walkability within the 38109 ZIP code to the convenience stores is depicted in Figure 4-10. Based on a 5 minute walking distance, it was estimated that approximately $2.5 \%$ of the population of 38109 are within the specified distance criteria.

Table 4-7 identifies names of the convenience stores on the map to assist in readability of locations and clear map visibility. Four convenience stores in the 38109 area were chosen to conduct field observation.

Field note \#13: 8/9/2014 - Location: Express Stop, Memphis, TN 38109 No fruits or vegetables are sold in this location. There is a cage around the cashier area. Beer and spirits are a prominent store feature in the freezer cases and on the aisles. Candy is also a prominent feature. Typical convenience store items, including chips, soda, cookies, candy, etc. is available.

Field note \#14: 8/9/2014 - Location: Hop-In, Memphis, TN 38109 No cage is present in this store. The store also sells bananas; the store clerk indicates that no other fruit is sold here. Beer is again a prominent feature in this store, present on both the middle aisle and in the freezer/refrigerator section. Typical convenience store items (chips, cookies, soda, etc.) are sold here.

Field note \#15: 8/9/2014 - Location: Safeway Food Mart, Memphis, TN 38109 This location advertises that they sell "cold cuts, groceries, discount cigs, hot food, gyros, fish, and barbeque (breakfast, lunch, and dinner)". Inside, they also sell hamburgers, chicken, and typical grocery store canned goods. Again, beer is a prominent feature within the store, offered in large quantities in the middle aisles as well as the refrigerator section. The grocery store clerk indicates that there are no fresh fruits or vegetables sold at this location.

Field note \#15: M\&A Grocery, Memphis, TN 38109

The outside of this building advertises that they accept EBT (Electronic Benefit Transfer) and debit, and that they sell pizza and wings. This is not a convenience store that sells gasoline. Inside, a large selection of beef and spirits are present. The food that was advertised is actually not sold at this location, indicated by the store clerk. He also indicates that no fresh fruits or vegetables are sold.

\section{Grocery stores - 38109}

Eight grocery store locations were identified within the area of 38109. These locations included Kroger, four Save-a-Lot brands; Super Valu Foods (similar to Save-aLot), Maxi, and Safeway Market. The degree of walkability within the 38109 ZIP code to the grocery stores is depicted in Figure 4-11. Based on a 10 minute walking distance, it was estimated that approximately $10 \%$ of the population of 38109 are within the 


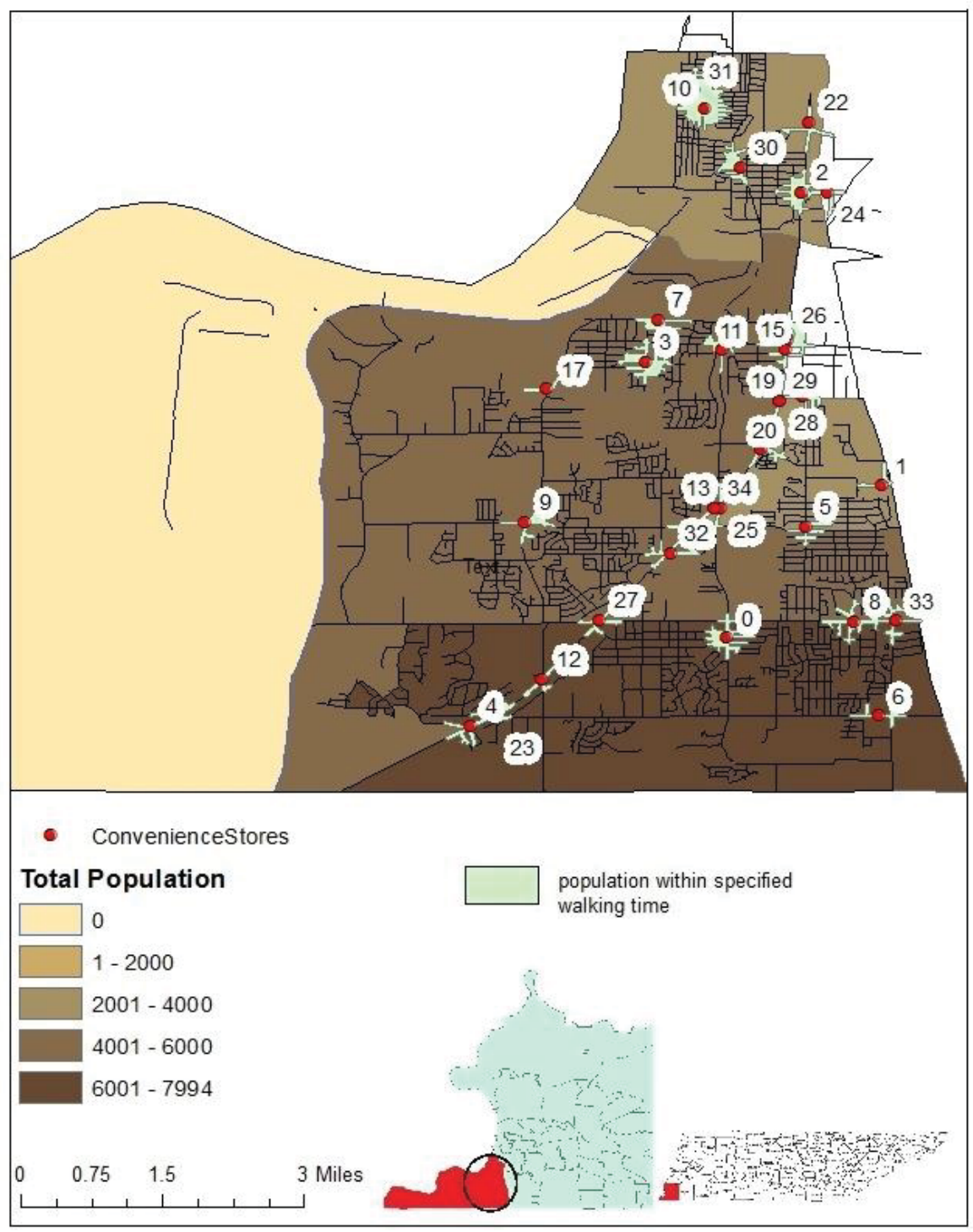

Figure 4-10. Residents of 38109 within 5 minute walking distance from area convenience stores. 
Table 4-7. 38109 convenience store identifiers and labels.

\begin{tabular}{|c|c|}
\hline Map identifier & Store label \\
\hline 0. & $\begin{array}{l}\text { Express Stop } \\
\text { Save Mart }\end{array}$ \\
\hline 1. & Exxon \\
\hline 2. & S Mart Market \\
\hline 3. & Марсо \\
\hline 4. & Pac n Snac \\
\hline 5. & $\mathrm{R}$ and $\mathrm{S}$ \\
\hline 6. & Caldwell's Quick Stop \\
\hline 7. & Fast Mart \\
\hline 8. & Tippy’s Market \\
\hline 9. & Silverage Sundry \\
\hline 10. & $\mathrm{BP}$ \\
\hline 11. & Pretti's Lucky Store \\
\hline 12. & Exxon \\
\hline 13. & Village Mart \\
\hline 14. & Mapco Convenience \\
\hline 15. & Walker Home Grocery \\
\hline 16. & N. Seven Market \\
\hline 17. & Gene's Stop and Shop Grocery \\
\hline 18. & BP Food Shop \\
\hline 19. & Stop N Save \\
\hline 20. & Walgreens \\
\hline 21. & Walgreens \\
\hline 22. & Dodge's \\
\hline 23. & Mapco Convenience \\
\hline 24. & Safeway Market \\
\hline 25. & Road Runner \\
\hline 26. & Exxon \\
\hline 27. & Citgo \\
\hline 28. & BP Food Shop \\
\hline 29. & Pack and Go \\
\hline 30. & Riverside Sundry \\
\hline 31. & Mapco \\
\hline 32. & Amoco \\
\hline 33. & On the Run \\
\hline
\end{tabular}




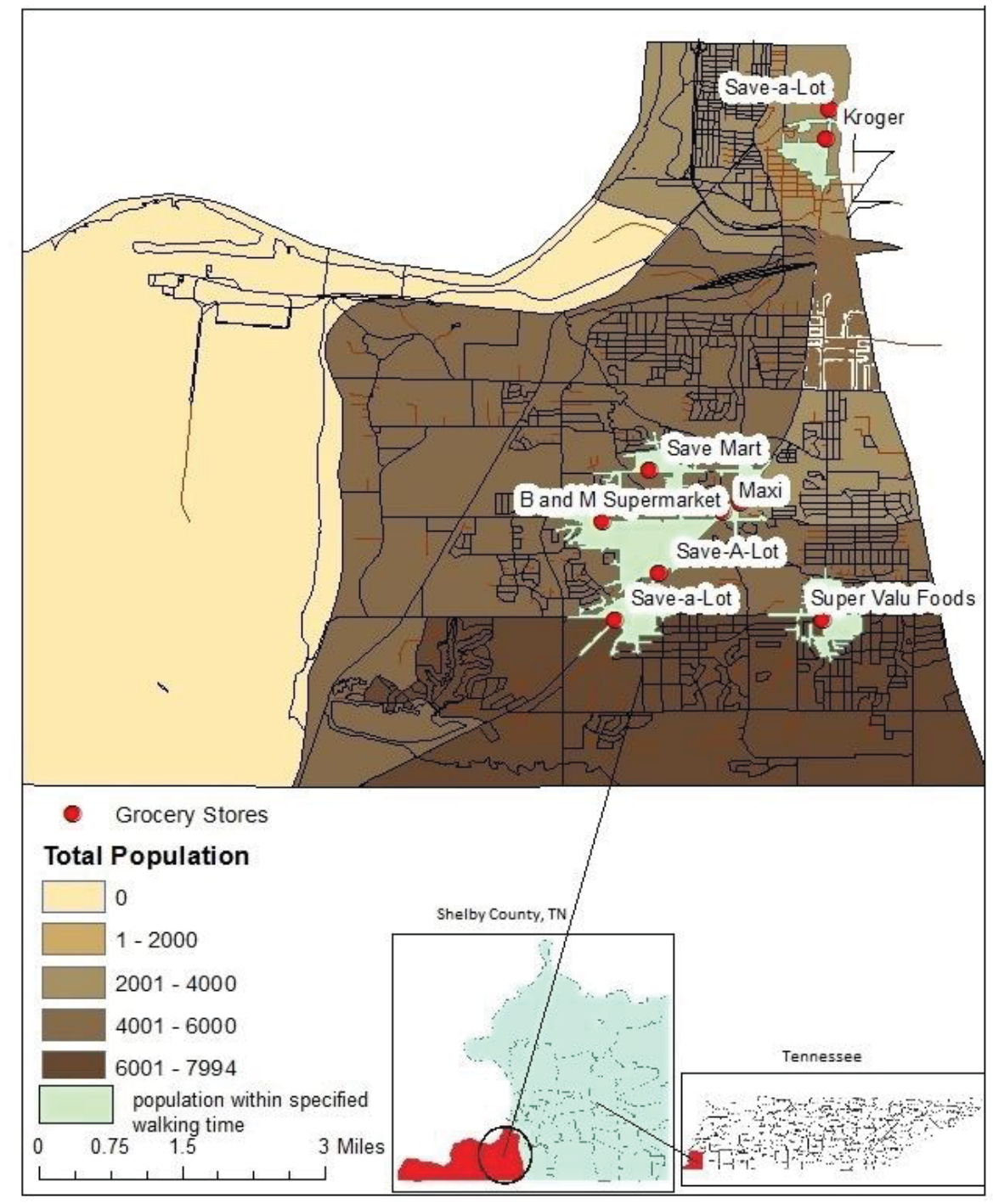

Figure 4-11. Residents of 38109 within 10 minute walking distance to area grocery stores. 
specified distance criteria. Grocery store quality was assessed via field exploration of two area stores, with observations documented. Two of the area grocery stores were chosen as representative of the grocery store selection in the area; Save-a-Lot has several franchises available in 38109, and found in other areas, and Kroger is a brand found in other areas as well. Kroger was chosen to determine whether or not the brand selection and quality was significantly different from what is typically found in Kroger in other areas of Memphis, TN, based on researcher experience. The proceeding field note lends insight into the field observation:

Field note \#6: 3/30/2014 - Location: Save-a-Lot, Memphis, TN 38109 Again, a turnstile located at the entrance of the store. Produce section appears to be of significantly higher quality compared to the 38106 counterpart. There also appears to be a larger selection Produce available for sale at this location included: banana, yellow onions, cabbage, celery, greens (collard, mustard, turnip), apples (golden, red, green), green bell pepper, red bell pepper, Idaho potato, sweet potato, mushroom, orange, tomatoes, grapes, and corn. Again, there also a large selection of pork, cakes and pies, with a limited lean meat selection. The leanest ground beef available is $73 / 27 \%$ fat content. No ground turkey, or lean cuts of chicken are available. No organic selections are available, again indicating that this may be representative of the brand. Although the quality is higher than the Save-a-Lot in 38106, there remains a limited selection of products when compared with the Save-a-Lot canvassed in 38122. In terms of accessibility, the proximal streets are long, high traffic areas as participants indicated. In general, the area seems less conducive to active transportation, considering that supermarket stores are not particularly close to residential neighborhoods. Furthermore, observations of the neighborhood reveal that there is a limited sidewalk network, as indicated by the study participants.

\section{Health facilities - 38109}

Four health facilities were identified within the area of 38109 depicted in Figure 4-12. The identified health facilities were Walgreens, Christ Community Health Services, Methodist Healthcare Wound Healing Services, and Shelby County Schools - SW Regional Health Clinic. Based on a 15 minute walking distance, it was estimated that approximately $6 \%$ of the population of 38109 are within the specified distance criteria.

\section{Parks - 38109}

Eleven parks were identified within the area of 38109. The degree of walkability within the 38109 ZIP code to an area park is depicted in Figure 4-13. Based on a 10 minute walking distance, it was estimated that approximately $6.7 \%$ of the population of 38106 are within the specified distance criteria. Five parks were chosen for field observation. The following field notes lend insight into park conditions within the 38109 ZIP code. 


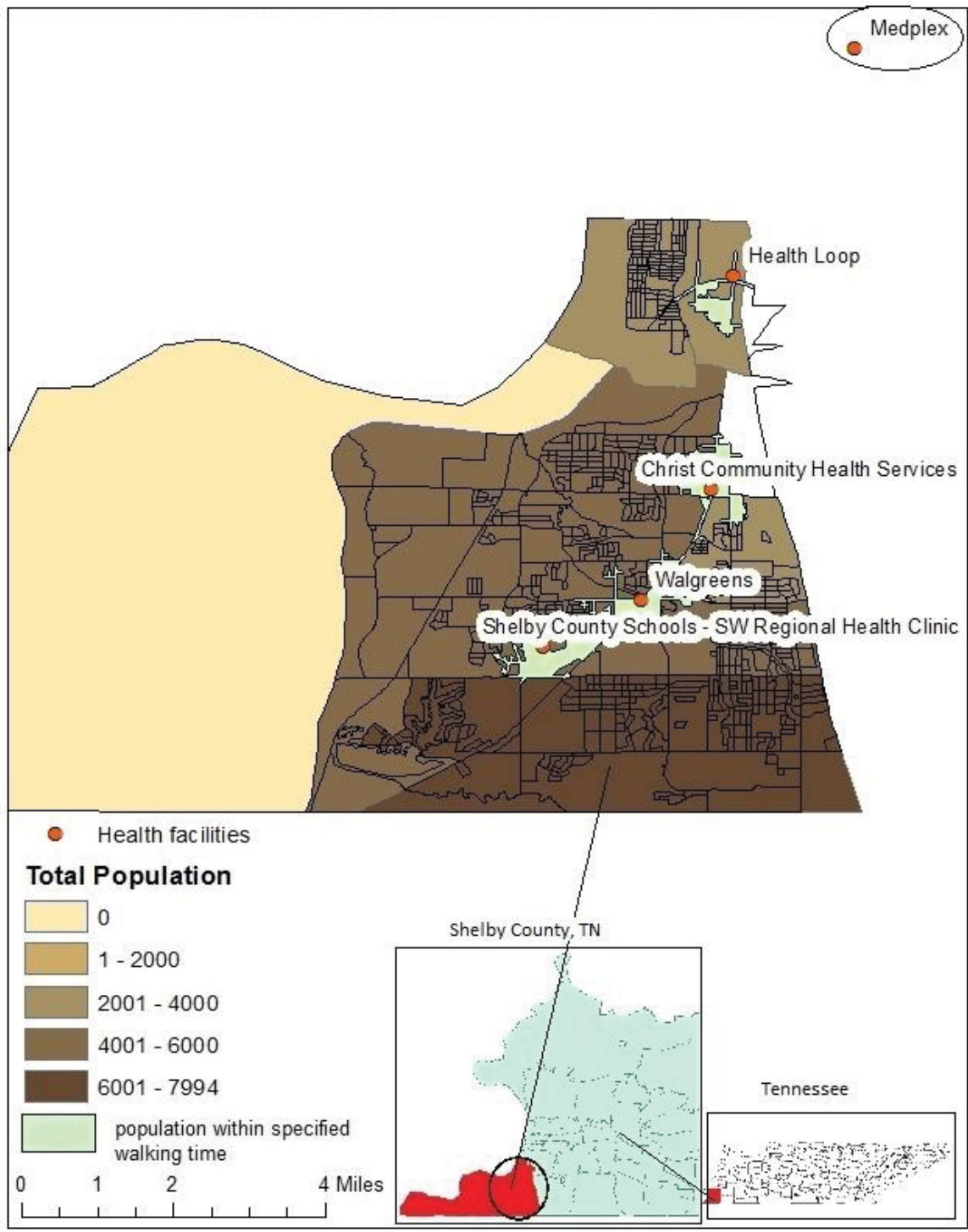

Figure 4-12. Residents of 38109 within 15 minute walking distance from area health facilities. 


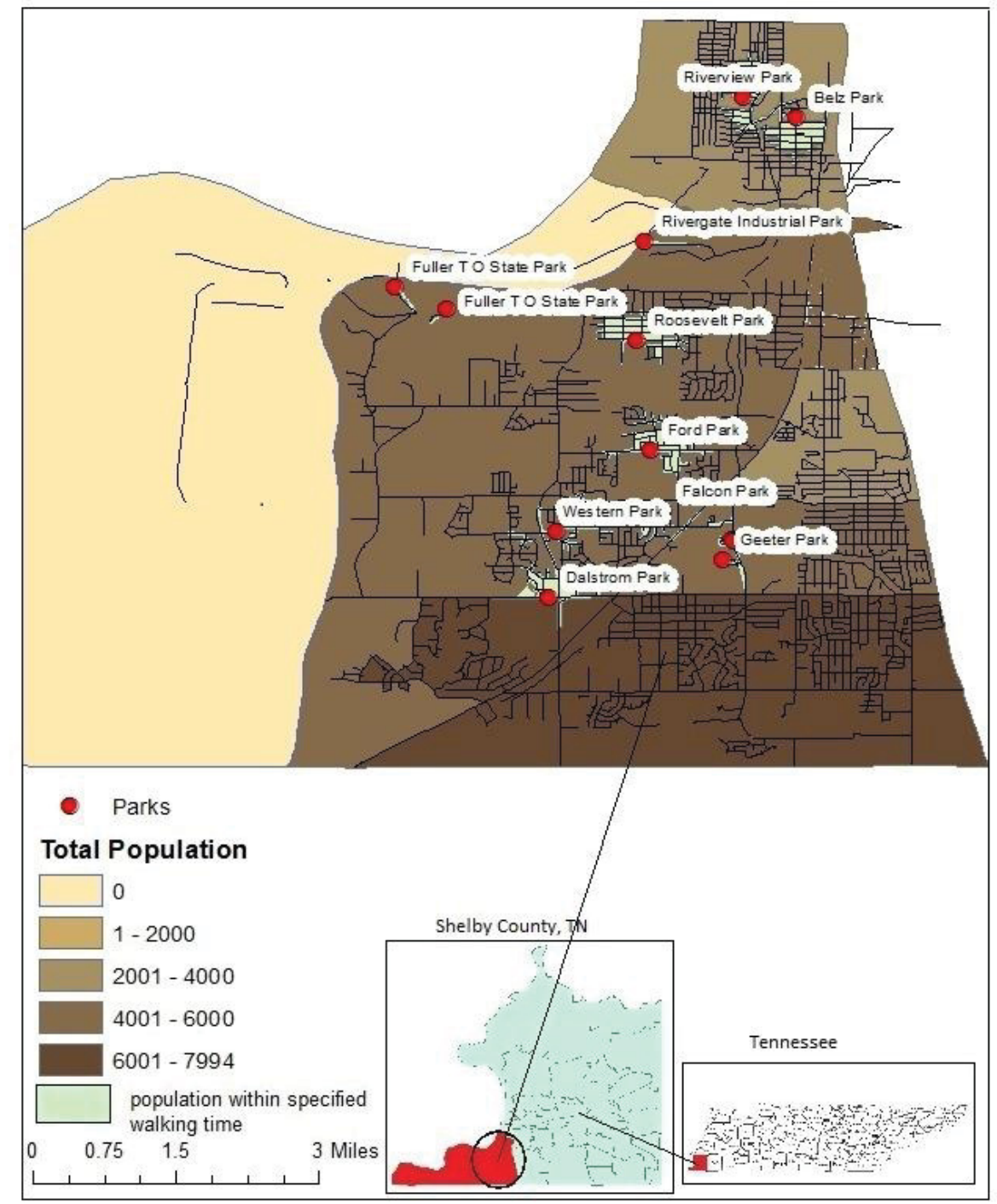

Figure 4-13. Residents of 38109 within 10 minute walking distance of area parks. 
Field note \#8: 8/9/2014 - Location: T.O. Fuller State Park, Memphis, TN 38109 No sidewalks were observed leading into the park, nor is the park accessible through transit - there are no nearby transit stops. Within the park, there is a playground, a picnic area, and a swimming pool, along with a basketball court, and batting cages. There appears to be a walking trail accessible as part of the park golf course. Geographically, this park is located in the western quadrant of the ZIP code. There are no observed residences located in nearby proximity; this seems to be a more isolated area of the ZIP code.

Field note \#9: 8/9/2014 - Location: Riverview Park, Memphis TN 38109 Riverview Park is the northern-most located park in 38109. Takes approximately 13 minutes to get from Fuller Park to Riverview Park. Sidewalks are present around this park, indicating accessibility through walking. The park is also located in a residential neighborhood - there are houses and two churches in the nearby area. In the park, there is a walking trail, a playground, a basketball court, and park benches. There are two men walking around the area. Although there are many qualities that could make the park ideal for engaging in physical activity, the overall intangible "feeling" however, is that the park may not be safe. Riverview Middle School is in close proximity to this park as well. There is a small play area for small children, a basketball court, and a walking trail available.

Field note \#12: 8/9/2014 - Location: Roosevelt Park, Memphis, TN 38109 This park is indicated on the map as the most walking accessible park in the 38109 ZIP code - more residents have walking access to this park than any other park in the area. It is also located in a central area of the ZIP code.

Upon area observation, it is noted that the area is actually a heavily and densely area. No walking trail or opportunities for activity are present.

Field note \#10: 8/9/2014 - Location: Dalstrom Park, Memphis, TN 38109 This park is located in the southern quadrant of the 38109 ZIP code. There is a playground, a picnic pavilion, and a walking trail. There are no sidewalks leading to this area, nor is there a transit stop designated for this area, but there are transit stops in close proximity. The park is large. There are residences also in close proximity to the park. The residential area near this park, however, has no sidewalks available.

Field note \#11: 8/9/2014 - Location: Belz Park, Memphis, TN 38109

Belz Park is located in a residential neighborhood. There are sidewalks leading to this park as well, and the park is tucked away behind an apartment complex.

\section{Transit stops $\mathbf{- 3 8 1 0 9}$}

There were 460 transit stops identified within the area of 38109 . The degree of walkability within the 38109 ZIP code to the area convenience stores is depicted in 
Figure 4-14. Based on a 5 minute walking distance, it was estimated that approximately $56 \%$ of the population of 38109 are within the specified distance criteria.

\section{Part 4. An Analysis of 38106 and 38109: A Comparison of Participant Reports and Field Observations of Major Environmental Characteristics that Affect Physical Activity}

The final part of this analysis will examine differences between the participant accounts and the environments of 38106 and 38109, along with noteworthy field observations used to supplement participant reports. Although both areas share similarly high rates of diabetes hospitalizations, and there are similarities between the two ZIP codes and in the participant accounts, there are also notable differences. Women relayed similar experiences in regards to sociocultural and economic influences. This is expected because the criteria used to recruit participants from each ZIP code was identical with the exception of the residential ZIP code. Additionally, both ZIP codes showed no available fitness facilities. However, the differences reported between the two specific environments were reported to impact the women and their physical activity and health management activities in different ways.

Walkability to resources identified as potentially contributing to weight loss and health maintenance in both the 38106 and 38109 environments was impacted by differing mechanisms, as reported by the study participants. The factor reported to most negatively affect decisions to engage in regular physical activity among women of 38109 was the lack of sidewalks in residential areas, which served to severely limit area walkability and decisions to utilize the environment for physical activity. Field observations of the 38109 ZIP did reveal that there are places within the area that do not have sidewalks; however, there are also places that do have sidewalk availability, but they may either be unkempt, display areas of limited connectivity, or are generally inconsistently present. Also, women indicated that the busy streets of 38109 were a deterrent to engaging in physical activity within 38109 .

In contrast, in the 38106 ZIP code, the most significantly reported deterrent to engaging in consistent physical activity was crime, thus by a different mechanism, affecting walkability. Environmental barriers reported to be present, however, included perceived unsafety of parks, a low quality grocery store, and poor quality community centers.

Field observation of parks in the 38106 area did demonstrate negative social indicators, including panhandling, an observation of public consumption of alcohol by park inhabitants, and substantial blight (in the form of abandoned houses, burned down houses, graffiti, litter, and overgrown lots) in nearby areas (although not all parks demonstrated these negative social indicators). Parks in 38109 were not as consistently observed to have negative social indicators that may deter physical activity; however, accessibility to 38109 parks was observed to potentially be problematic, especially with noted limited sidewalk accessibility and general isolation away from residential areas. 


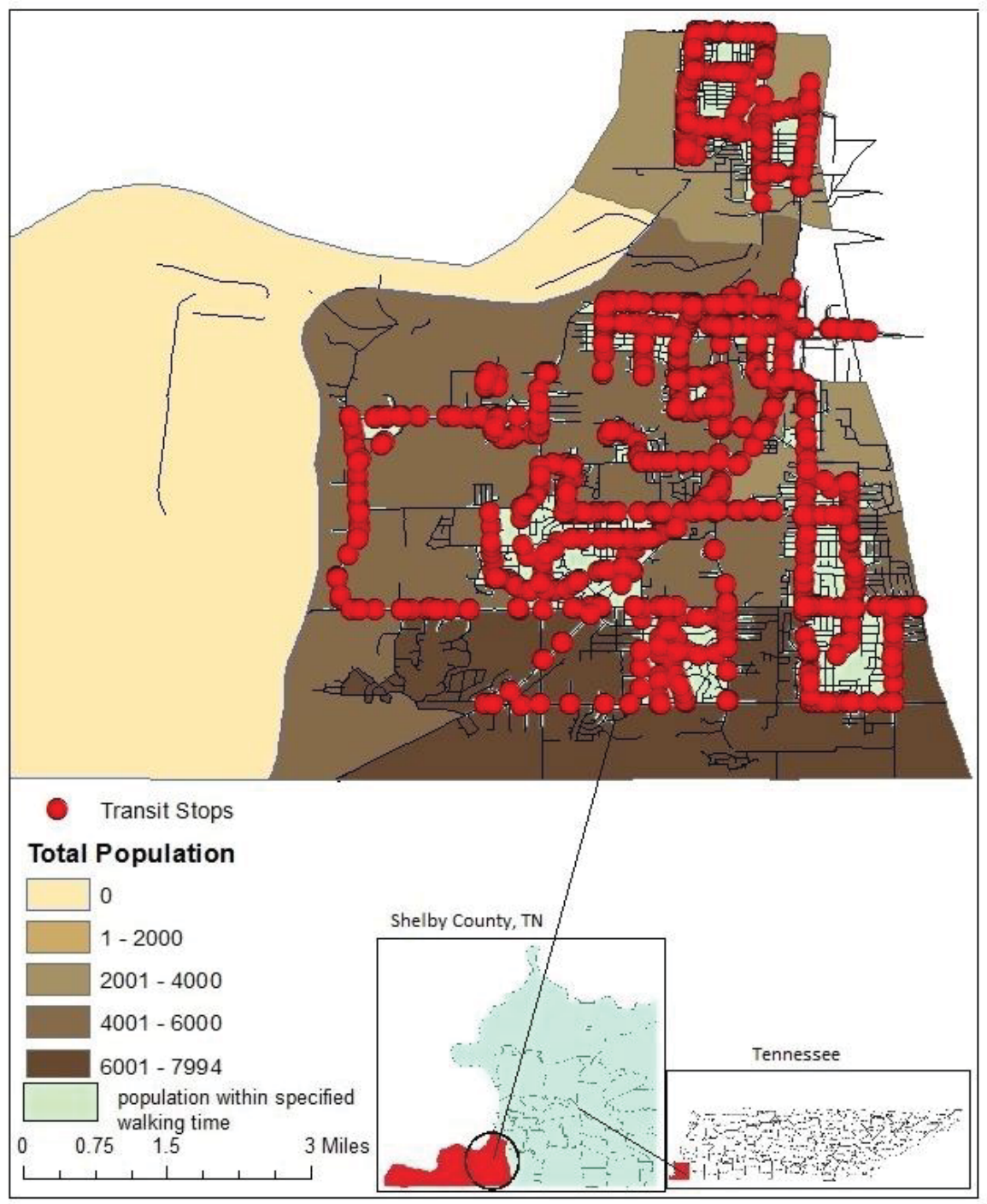

Figure 4-14. Residents of 38109 within 5 minute walking distance from transit stops. 
Grocery store quality also differed between 38106 and 38109. Save-a-Lot is a deep discount supermarket chain that describes itself as a store that brings "discount groceries to neighborhoods across the US." They go on to say, "as one of the nation's leading extreme value carefully selected assortment grocery chains, we deliver our customers terrific savings on discount groceries, up to $40 \%$ compared to conventional grocery stores. The time-saving set-up of Save-A-Lot stores, combined with high quality discount groceries, allows us to offer great food at great prices from great people, every day." Although the store advertises that they sell high quality produce, participant reports and field observations particularly from the 38106 location suggest that this is not the case. Participants from 38109, however, suggested that the produce quality was not necessarily a problem in 38109 - accessibility (and particularly, quick accessibility) was more of an issue. Table 4-8 gives a summary of major environmental influences reported by the study population to affect physical activity and health maintenance. 
Table 4-8. A comparison of major environmental influences reported that affect physical activity and health maintenance in 38109 and 38106 ZIP

codes.

38106

Limited fitness facilities

Perception of unsafe

community centers

Perception of unsafe parks

Substantial reports of stray animals

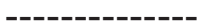

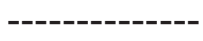

Crime

Low quality grocery store

High numbers of corner stores with no produce for purchase
38109

Limited fitness facilities

Limited utilization of

community centers

Limited walkability to area

resources, no sidewalks

Long street network, limited "quick"

accessibility to area

resources

Grocery stores of higher quality

High numbers of corner stores with limited produce for purchase 


\section{CHAPTER 5. DISCUSSION OF FINDINGS, POLICY IMPLICATIONS, STUDY LIMITATIONS, FUTURE DIRECTIONS, AND CONCLUSIONS}

\section{Introduction}

Several studies have quantitatively examined causal conditions associated with type 2 diabetes mellitus and have found it to be a complicated, multifactorial disease. Not only may it be the consequence of genetic susceptibilities triggered by individual actions as some research suggests; it is also influenced by social, cultural, psychological, and environmental factors. According to current literature, rates of type 2 diabetesrelated mortality and morbidity are significantly higher in African American communities and because of this, it is an ideal community to study to gain knowledge of the health experiences of at risk minority communities.

In Memphis, TN, two areas of the city (38106 and 38109) have the highest rates of diabetes hospitalizations. One goal of this study was to shed light on factors that encourage and discourage weight loss and health maintenance; another goal of this study was to understand sociocultural and economic factors that may be driving these hospitalization rates particularly among women, and to understand the experiences in their own words. An issue brief published in 2012 by The Methodist Le Bonheur Center for Healthcare Economics in association with the University of Memphis also suggested that African American women are the most vulnerable group at risk of a potentially avoidable hospitalization in Memphis (University of Memphis, 2012). This study provided a qualitative analysis of the aforementioned environments with noted high rates of diabetes hospitalizations, and sheds light on many factors that may be specifically contributing to these high rates among African American women in these areas. Subsequently, knowledge gained from this specific study using the African American diabetes management health experiences among women can be used as a springboard to explore additional specific factors, thus assisting in a better understanding of experiences with diabetes in Memphis, TN.

\section{A Discussion of Sociocultural and Economic Factors that Affect Health Maintenance, Weight Loss, and Physical Activity among African American Women in the 38106 and 38109 ZIP codes}

Several factors were identified as both negatively and positively impacting study participants' ability to maintain their health, engage in regular physical activity, and lose weight to effectively manage their diagnosed diabetes; however, these factors were more often negative, rather than positive, in nature. Previous literature has suggested influences on diabetes self-management behaviors of African American women may be best understood from a sociocultural and family context and include factors such as spirituality, feelings of dietary deprivation, and physical and emotional "tiredness" as well as fear of diabetes complications (Samuel-Hodge et al, 2000). Sociocultural factors impacting diabetes management gauged from the study participants included nutrition 
barriers, extensive generational diabetes, and crime; each of these factors impacted the overall management of type 2 diabetes among the study population in various ways. Nutrition was a multidimensional barrier that was widely reported to contribute to difficulty and inconsistency in diabetes management. When women in the study were asked about factors that influence weight loss and diet control, weight management, and exercise, all women responded that not only was nutrition a primary barrier, it could also be a positive influence when sufficient dietary changes were consistently made, albeit difficult to accomplish. Family was also an important consideration when examining dietary behavior; women suggested that making changes to their family's dietary habits was difficult to accomplish and made making nutrition and dietary changes difficult as well.

Notably, supermarkets found in the study ZIP codes demonstrated significant differences in the quantity and quality of healthy food items, especially when compared to an area with a more diverse demographic composition. With respect to quality, this difference was most substantial in the 38106 ZIP code. Differences within food environments highlight that areas with large African American populations may have unequal access to healthy and high quality food sources. Nutrition may be impacted not only through social conditioning and social customs, but the availability of items sold within area stores; the economic concept of supply and demand may also drive availability of items for purchase and store assumptions regarding consumer choice. Ideally, store quality should be standard regardless of area location. However, as reported by study participants, although being in a "black" neighborhood should have no influence on the quality of items sold, whether a turnstile is located at the store entrance to prevent theft, or whether specific foods are or are not made available, the perception is that area demographic composition impacts store quality. Field observation supported this perception as well - across areas, the supermarket brand Save-a-Lot demonstrated differences in available produce and inconsistent store quality. This, in turn, could be detrimentally affecting the nutrition culture of the area, and impacting women's ability to easily manage their diabetes through diet.

Generational diabetes was extensively reported among women in the study, potentially affecting global views and realistic perceptions of effective diabetes management. Women in both 38106 and 38109 reported that they had mothers, fathers, sisters, brothers, cousins, and other family members affected by diabetes; additionally, both negative and positive effects within social observations of diabetes were reported. In the positive dimension, some women were inspired by family members' experiences with effective management, or had a fear-based response to the complications observed from others' experiences, prompting the women to desire to more effectively manage the condition. In the negative dimension, many women perceived that because their family and friends had challenges in managing the condition, they would therefore, themselves have difficulty; this sentiment was the more predominant perception among the women. Many of the study participants reported that they did not observe family members successfully managing the condition. Not having an example of diabetes being effectively managed through exercise and diet by known social networks may create the perception that the condition cannot be adequately managed in this way, and that in some 
ways, the disease course is inevitable. Fatalism within the context of the African American diabetic experience has been explored, specifically characterizing interactions, meaning attached to interactions, and decisions to adopt self-management behaviors (Egede \& Bonodonna, 2003). With this perception, and family members in close proximity, diabetes hospitalizations in 38106 and 38109 may be fueled and propagated by limited social examples of adequate and effective diabetes management. An explanatory social construct of the nature of poorly managed diabetes that incorporates the significance of extensive generational diabetes within a self-management behavioral context has only been explored in a limited capacity in the literature. Harrison et al (2003) suggested that a family history approach may be a promising public health direction needed to combat the growing diabetes epidemic. Further, a qualitative study in Oaxaca, Mexico uncovered the detriment of attributing type 2 diabetes to "family history" within rural communities (Everett, 2011).

Crime served as a major barrier to physical activity in the 38106 area, reported by many of the study participants. The literature has previously suggested that physical environment factors, including crime, has consistent associations with adults' decisions to engage in physical activity (Humpel, Owen, \& Leslie, 2002). Contextually referring to women and diabetes management, the crime environment, particularly in 38106, was instrumental in the decision-making processes of women with limited social connections in their residential community. Although typical diabetes management protocol includes physical activity, the decision to integrate this healthcare advice as a daily management tool was minimal among women who had limited social ties; these women were more affected by their perception of crime in the area. Ecologic models suggest that at the organization and public policy level, the environment for physical activity, including perceived safety, affects rates of obesity. Among the study population, crime, integrated within a sociocultural context, affected levels of physical activity and decisions to utilize the environment for physical activity. Social connectedness and the degree to which women were integrated within their communities impacted decisions to engage in physical activity; more specifically, more residentially-socially integrated women more often reported engaging in physical activity despite their perceptions of crime. These behavior- and context-specific decisions can potentially be integrated into ecologic explanatory models of African American women with diabetes within a low income setting. Humpel, Owen, and Leslie (2002) also suggested that further development of ecologic and environmental models in context-specific ways can potentially assist in advancing the understanding of the association between environmental attributes (including crime) and physical activity.

Economic concerns present among the study participants included lack of adherence to management protocol because of concerns regarding cost of medications and medication equipment, the perception that lack of health insurance contributes to low quality of care by providers seen, and circumstances surrounding women's financial resources combined with perception that eating healthily is more expensive. Cost is a well-documented barrier in the literature noted to contribute to poor compliance with self-monitoring and poor adherence to treatment (Marshall, 2005). Perceived quality of care and possession of health insurance has also been explored within the context of race 
(Perez, Ang, \& Vega, 2009). As stated previously, the food environment potentially plays a significant role in study participants' ability to engage in healthy eating and has also been explored (Galvez et al, 2007). Living within a generally low-income community, where demand is directed toward more unhealthy food options, along with low proximal accessibility to high quality food sources, individual- or interpersonal-levels of the socioecological model may interact with organizational-level factors facilitating poor outcomes. Differences in food resource quality, particularly in 38106, may exacerbate the health status differences between residents of this area and surrounding areas.

Sociocultural and economic influences within the study areas primarily were found to discourage successful weight loss, health maintenance, and physical activity among low-income African American women interviewed from the 38106 and 38109 areas. Social-level factors, including women's family accepting change, high rates of crime, and generational diabetes, were found to be an important determinant in selfmanagement decision made by the study participants. In this study, it was shown that relationships established with neighbors and family can contribute to more consistent self-management behaviors. Implications and impact of social connections on individual functioning and well-being of women with type 2 diabetes suggests that social-level factors are very integral in their decision making processes. Further, what was found among the women in the study was that socially construed explanatory models are intimately connected with generational diabetes. "People" and observations of people in social environments are an important determinant in considering how one may care for themselves and their health. Sociocultural factors seem to create expectations independent of personal circumstances. For example, among women in the study who observed family members, friends, and neighbors with type 2 diabetes, there were already preconceptions of how the disease would progress, what comorbidities would occur, and what followed was a degree of resignation. In other words, to address the health of one person without addressing the health of all observed influences may be less impactful than addressing the group as a whole. Sociocultural factors were found to be impactful to women's ability to self-manage their diabetes consistently and effectively. Economic factors, though reported as integral in the decision-making process, were less emphasized among the participants when compared to sociocultural and social-level factors in selfmanagement behaviors.

\section{A Discussion of Environmental Factors that Affect Health Maintenance, Weight Loss, and Physical Activity among African American Women in 38106 and 38109}

The environment was explored within the context of perceptions regarding characteristics of the physical environment and resources utilized to maintain health; and accessibility and walkability of the environment as perceived by study participants. Positive features of the environment that were mentioned by study participants included park availability and mobile farmer's markets, reported by women in 38106. Among women in both 38106 and 38109 , the main discouraging influences reported were low grocery store quality in 38106, and the lack of sidewalks in 38109. A summary of the chief environmental perceptions from 38106 and 38109 suggests that environmental 
factors may impact women's ability to maintain their health in more subtle and long-term ways. Limited sidewalk availability of 38109 residential areas serves as a physical activity deterrent in this area. Low quality produce present in 38106 , leaves women in 38106 searching for other options with regard to fresh fruit and vegetable availability. Participants did not readily note using the South Memphis Farmer's market, but noted utilizing mobile markets more frequently.

In the literature, supermarkets have been identified as components of the physical environment that may influence food choices, and more distal outcomes like obesity, by increasing the availability of healthy foods. Also in the literature, it has been noted that availability and accessibility to food sources may play a significant role in dietary quality and food choices and therefore has an important role in regulating risk for disease (Galvez et al, 2007). In the long term, poor store quality options within the 38106 area, particularly among persons with limited mobility and limited transportation, could affect diet quality, the nutrition culture and, more distally, diabetes outcomes including rates of hospitalizations. Additionally, a high number of corner stores in both 38106 and 38109 was also found through Geographic Information Systems; the disparate prevalence of urban corner stores in low-income and high-minority communities has been well documented and has also been noted in the literature to contribute to significantly higher energy intake, potentially exacerbating obesity rates in urban communities (Borradaile et al, 2009).

Also documented in the literature, urban sprawl (as seen in 38109 through limited sidewalk connectivity and long street networks) results in low-density communities reliant upon heavy automobile usage and has been implicated in rising obesity in several studies; additionally, it has been linked to physical inactivity, lack of social capital, chronic disease, and private-vehicle commute distances and times (Ewing et al., 2003; Kelly-Schwartz et al., 2004). The high degree of urban sprawl seen within 38109 may distally contribute to poor diabetes outcomes by discouraging physical activity, as reported by study participants. As one woman said, "We don't have sidewalks. The street is unsafe, and there are no sidewalks, so that in itself is a danger. We have several neighbors including myself, calling and complaining about [the lack of] sidewalks. And there is a park not far away but... if you don't have transportation how would you get there."

Resources identified as potentially contributing to health maintenance, weight loss, and physical activity among women in 38106 and 38109 included transit stops. A research brief published by Active Living Research on active transportation posits that people who utilize transit stops are less likely to be obese than adults who did not use transportation; additionally, proximity to public transit stops is linked to higher transit use and higher levels of physical activity (Active Living Research, 2009). However, crime was a major deterrent seen within 38106 that may preclude and overrule transit stop use in the absence of residential community social ties. The degree of accessibility to transit stops by residents of both ZIP codes is $56 \%$ for 38109 and $81 \%$ for 38106 , suggesting transit stops may encourage physical activity in the absence of crime perceptions. Overall, the social environment may play a key role not only in the resource environment 
available, but in what is then seen within the context of physical environment. These factors play a role in influencing whether or not the physical environment is utilized for physical activity. Figure 5-1 is a potential explanatory model, developed through the conduct of this study that depicts influence levels as seen within the study population. This figure partially explains how the social environment is a major decision-making point among the women of the study in regards to outdoor physical activity and health maintenance. The figure also details how social interaction can affect both quantity and quality of resources within communities, which can then have an effect on the physical environment of communities. Overall, the decision-making processes in regards to physical activity, weight loss, and health maintenance are a complex web that may ultimately be determined by the quality of the social environment, particularly in lowincome communities.

The social environment is thought to be comprised of not only an individual's families (both immediate and extended families), but also known neighbors, coworkers, and friends. Also comprising the social environment are unknown neighbors, and residents of the community who may influence the "character" of a community. A third level of social environment are from those outside the residential area who influence decisions that affect the environment, and can include business owners who decide to (or decide not to) open businesses within the community, and policy makers who make decisions for people within the community concerning assets and aspects of the physical environment that may be improved.

Socio-environmental characteristics then may affect the resource environment. The resource environment can include those assessed within this study such as health care, schools, grocery stores, parks, transit stops, community centers, convenience stores, and farmer's markets - these are the places that people of the community can frequent to gather information and receive services to meet day-to-day needs. In this study, the quality of resources, the quantity of resources available, and the demand for resources may be impacted by socio-environmental characteristics - by the people in the community who create expectations and facilitate the demand for such services, impacting collective perception not only of the persons within the community, but also persons outside of the community.

The collective perception of persons within and outside of residential areas may affect resource availability, timing of resource offerings, and decisions regarding where resources will be, affecting accessibility within the residential community. The physical environment, including sidewalks and the degree of walkability within communities, presence or absence of stray dogs, and the presence or absence of blight, trash, overgrown grass, etc., which may deter or encourage physical activity can be affected by the quality and quantity of resources available. The presence of businesses, primarily determined by demand and the economics of the community, may also be influential in driving the appearance of the physical environment. Also significant may be the degree to which the residents of the community utilize the environment for physical activity (both actual and perceived utilization). 


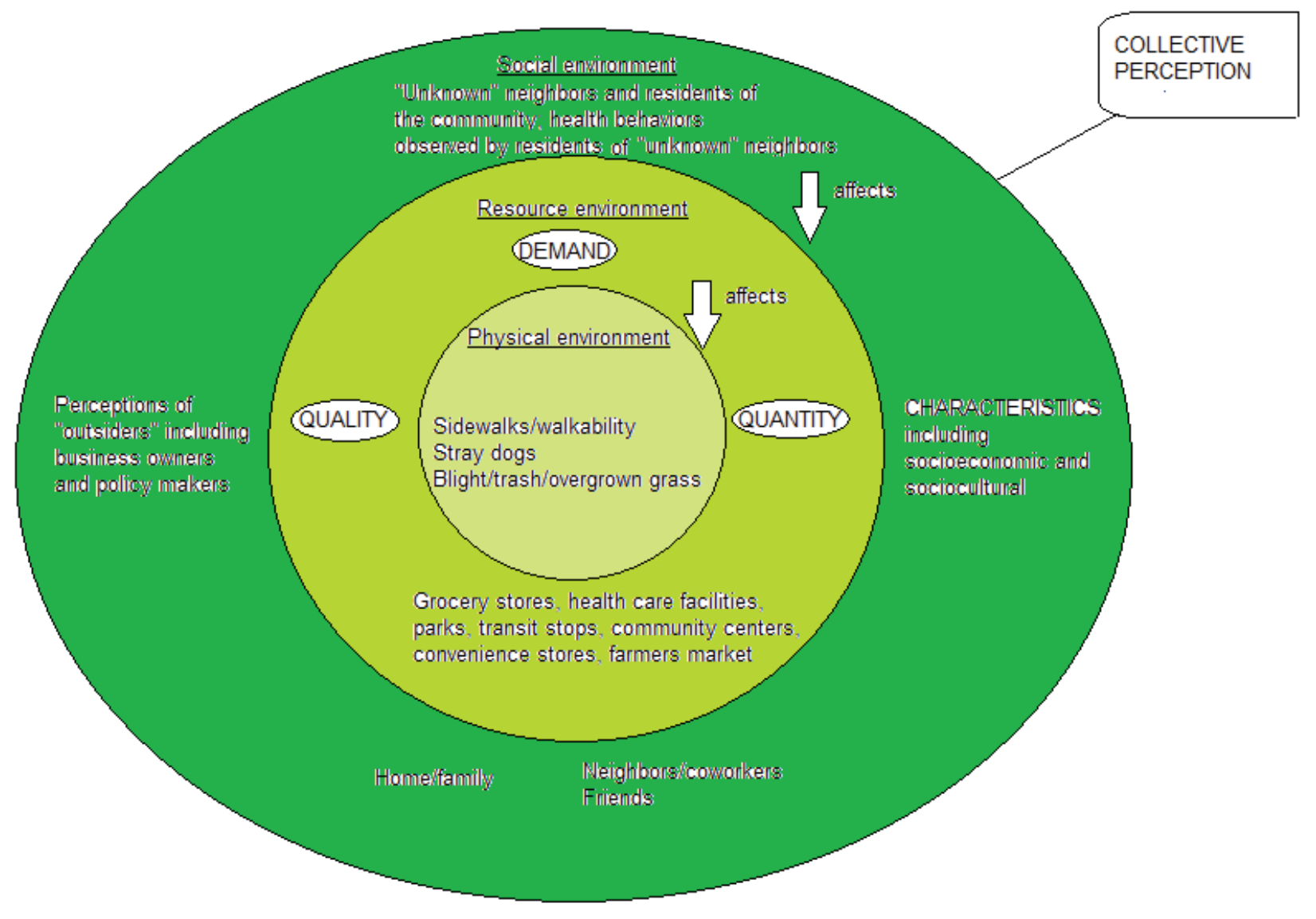

Figure 5-1. Potential determinants of physical activity and health maintenance within the study population. 
These various factors together potentially influence residents' individual decisions on whether or not to utilize the environment and engage in healthy behaviors, both from an individual frame and from a collective perception of social norms within the community environment.

\section{Policy Implications}

In Memphis, TN, there have been several initiatives particularly aimed toward redeveloping urban environments and improving quality of life (City of Memphis, 2014). The City of Memphis states that "on April 29, 1998, the Tennessee General Assembly enacted the Community Redevelopment Act of 1998... for the purpose of improving the quality of life through the prevention and elimination of slum and blight and providing affordable housing for low to moderate-income families within Memphis and Shelby County" (City of Memphis, 2014). Additionally, several small development agencies within Memphis have also implemented or initiated projects with the purpose of attracting "more people to Memphis and Shelby County with a vibrant Downtown that is densely populated, authentic, mixed-use, walkable, clean, safe, and fun" (Downtown Memphis, 2014). Memphis' Renewal efforts have aimed to improve local services in selected areas, to reduce crime, to reduce government requirements, and to involve community partners, including the Center City Commission, the City of Memphis Administrations, the Memphis Housing Authority, the United Way of the Mid-South, and the Workforce Development Agency (USHUD, 2014). Redevelopment efforts in other areas of the country have proven to be effective; however, some have questioned the viability of an urban redevelopment model that relies on small community development corporations over the long-term (Stoecker, 2008).

In 38106, several community development corporations serve to provide "services in areas of housing, economic development, education, and advocacy" (The Works, 2014). Comprehensive public works projects dedicated to safety and the elimination of blight may distally impact diabetes outcomes through the encouragement of physical activity. Additionally, the Memphis, TN police department has enacted several initiatives to reduce the overall crime rate in Memphis. Retail food inspections are required by Tennessee state law at least once every six months to "ensure compliance with all laws and regulations relating to food storage, facility sanitation, and safety" (Department of Agriculture, 2014). Compliance standards are related primarily to food protection and temperature, employee hygiene, water and sewage services, toilet and hand washing facilities, waste disposal, pest control, cleaning and sanitization, maintenance of floors, walls, and ceilings, sufficient lighting and ventilation, and proper storage and display of toxic materials (Department of Agriculture, 2014).

This study demonstrated the need for improvement of quality standards across the same store brand; these quality standards should not differ in demographically

dissimilar areas. Standardizing differences in store quality regulation through more strict compliance standards may affect health outcomes in the 38106 area over the long-term. In 38109 , a comprehensive public works project dedicated to increasing sidewalk 
connectivity may be necessary to encourage residential physical activity outdoors. Walkability and public health campaigns have been shown to be effective in promoting residential physical activity; conversely, disconnected street networks are associated with auto dependence and negatively associated with walking and transit use (Frank et al, 2006). Additional public health initiatives targeted toward the 38106 and 38109 areas to confront the substandard conditions mentioned within this study should aim to create healthier environments conducive to residential active living.

With regard to stray animals, the most specific policies detailed by the City of Memphis are rabies control policies, which have been detailed by the Memphis and Shelby County Health Department. These policies detail ordinances and laws requiring licensing for pets, as well as procedures following dog bites. According to County Ordinance 82-3-34 and Tennessee State Law, T.C.A. 68-8-104, owners of dogs in Memphis and Shelby County are required to "have their dog licensed yearly and must present proof of rabies vaccine at the time of issuance. Cats are not required to have a license, with the exceptions of residents in Collierville and Germantown. Each of these municipalities has City Ordinances that require cat licensure in addition to the vaccination. Failure to license and vaccinate dog or cat could result in being issued a citation to appear in General Sessions Court, and result in fines"(Memphis and Shelby County Health Department, 2014). Further, according to Tennessee State Law, T.C.A 68-8-111 and Shelby County Ordinance 82-3-46, "any dog biting a human, whether vaccinated or not, [must] be held in quarantine for 10 days..." (Memphis and Shelby County Health Department, 2014). However, the City of Memphis indicates that there are no specific policies and procedures detailed to streamline the identification, control, and capture of stray animals. Because stray animals are such a prolific problem particularly in the 38106 ZIP code and can impact physical activity efforts, more standardized animal collection and identification policies should be specified.

Finally, resources allocated toward park safety and the community centers of 38106 and 38109 may impact the degree of physical activity and positively impact the health of those with type 2 diabetes in these areas. The study findings showed that the City of Memphis does not efficiently allocate financial resources to the community centers of 38106 particularly; this is a missed opportunity to potentially incorporate health education for the widespread diabetic population of the ZIP code. Additionally, efforts to allocate resources to more frequently patrol the parks in 38106 and 38109 by the Memphis Police Department can encourage safety and park use for physical activity.

\section{Limitations}

Several limitations were identified throughout the conduct of this study. Many pertinent limitations were listed in Chapter 1. As previously indicated, the very nature of this qualitative study prevents generalizability. The scope of the analysis focused on exploring the experiences of women in 38109 and 38106 ZIP codes with regard to type 2 diabetes, and their weight loss, physical activity, and health maintenance efforts. The sample of 20 urban, low-income African American women recruited for this study does 
not necessarily represent the views of all African American women with similar demographic characteristics. Therefore, findings are not necessarily transferable. Also, the purposeful sample, for this study, was defined as meeting a specific set of criterion and is more representative of selective sampling (Coyne, 1997), where recruitment criteria was shaped based on the categories of age, gender, diagnosis, a visit to The Medplex Outpatient Center, being African American, and residing in one of two ZIP codes. Though variation across women's experiences was observed, thematic saturation controlled for differences in the main concepts regarding weight loss, utilization of the environment for physical activity, and health management. Further, member checks conducted with six of the study participants assisted in verifying the accuracy of the analysis results.

Development of the interview guide was a cyclical process, and thus, many pertinent questions were not asked during the initial interviews. Although through follow up, questions not asked before were asked of participants recruited at the beginning of the study, participants may have already been biased toward questioning from previous questioning. To control for this, participants were asked what they remembered from the previous conversation. Additionally, many questions were asked repeatedly in varying ways to obtain the richest and most detailed information possible. Further, generalizability is compromised by limitations within the study design that prevent the determination of specific causes of phenomena observed within target ZIP codes.

Additionally, without a comparison group to draw out true differences within reported experiences, it is difficult to state whether or not phenomena determined to exist within the ZIP codes of interest (or within the population of interest) are distinct from other areas of the city or groups of people in the city. Although several influences were found within the study population that were useful and interesting to consider, it cannot be said that this is specific to either the ZIP code communities or the people who reside within them. Specific characteristics targeted by the study design can only speak in terms of the study group, and not the population in general.

\section{Future Research Directions}

Future research should potentially focus on a comparison of women in the 38109 and 38106 ZIP codes with similar women in demographically more diverse areas, as well as areas with low rates of diabetes hospitalizations, to compare their experiences with the environment, as well as socioculturally and economically. This may assist in parsing out environmental influences driving diabetes management difficulties among residents with diabetes within the 38106 and 38109 ZIP code areas. Additionally, the extensive generational diabetes seen within this study is interesting to consider and should be further explored, in a social context. Many studies suggest that generational diabetes may be the result of genetic predisposition; however, insight provided by this particular study indicates that social interaction and social observation may be more impactful when considering diabetes self-management behaviors. The social implications and consequences of generational diabetes have not been thoroughly explored in the 
literature. Not only does this provide a potential explanatory model for women in the study areas, this may apply to the global scope of social interaction and social functioning while having type 2 diabetes.

Future research should also further explore the cases of women who are extremely successful and proactive in managing their diabetes. Many of the women interviewed in this study had a degree of resignation regarding their condition; drawing out and specifically seeking women who are more proactive and optimistic about managing their diabetes may inform potential intervention strategies from a healthcare provider perspective. Finally, policy that seeks to impact urban development within areas that demonstrate high rates of diabetes hospitalizations should be thoroughly evaluated to not only determine the economic effects of revitalization, but the health effects on residents in these areas as well.

Finally, relationships, impact of relationship quality on health status, the impact of generational diabetes on diabetes management, and the impact of social relationships on diabetes management should be explored in future research. The quality of relationships within the context of this study was found to be an important factor in shaping women's decisions to engage in physical activity and weight loss efforts consistently. Both qualitative and quantitative analyses can be utilized to explore this phenomenon; for example, National Health and Nutrition Examination Survey (NHANES) data collected by the Centers for Disease Control (CDC) has several variables that explore various social determinants on health status, including time spent eating together as a family, frequency eating at restaurants for social reasons, whether or not any relatives are attempting to lose weight, and blood relatives possessing specific chronic conditions.

\section{Conclusion}

Education and community awareness play a significant role in the perception of type 2 diabetes as a social disease. As seen in the perception of risk, formation and creation of explanatory models, and the likelihood of engaging in preventative activities, sociocultural values play a significant role in establishing African American women's health perceptions. Additionally, various populations may place value on different things depending on economic, environmental, and cultural circumstances. Within low-income populations, social capital and connections may be inextricably tied to the degree to which the study participants self-manage their diabetes. Among the study population of low-income diabetic women, the quality of the relationships in their lives -- community relationships, familial relationships, and individual self-efficacy as a function of physical and social environmental factors -- contributes substantially to predictive determinants of environmental utilization for physical activity. However, the environment is also an important consideration and, combined with an assessment of sociocultural and economic influences, highlighted several key considerations of residents within the 38106 and 38109 ZIP codes. Particularly, understanding the importance of social roles (both acted and observed) of African American women in this study may expose factors that impact their vulnerability toward experiencing a potentially avoidable hospitalization. 
The environment does play a crucial, although secondary, role to social capital, the social environment, and the ties between these and self-management of diabetes. Although the initial, primary target of this study was to evaluate how perceptions of the physical environment affect physical activity among the study participants, in conducting and completing the study, it was found that the quality of relationships established (within the framework of family and community) can overrule environmental circumstances and encourage physical activity. In the absence of quality relationships that encourage positive health maintenance, the social environment of the community, along with the physical environment, can determine the degree of selfmanagement seen in individuals and their utilization of the environment for physical activity. 


\section{LIST OF REFERENCES}

Abley, Stephen. (2005). "Walkability Scoping Paper". Retrieved May $2014<$ http://www.levelofservice.com/walkability-research.pdfs

Ainsworth, B., Wilcox, S., Thompson, W, et al. (2003). Personal, social, and physical environmental correlates of physical activity in African American women in South Cfarolina. American Journal of Preventive Medicine, 25(suppl I), 23 - 29.

Allison, D. B., Fontaine, K. R., Manson, J. E., Stevens, J., \& VanItallie, T. B. (1999). Annual deaths attributable to obesity in the United States. JAMA, 282(16), 15301538.

Allison, D. B., Zannolli, R., \& Narayan, K. M. (1999). The direct health care costs of obesity in the United States. [Research Support, U.S. Gov't, P.H.S.]. American Journal of Public Health, 89(8), 1194-1199.

American Academy of Pediatrics Committee on Pediatric Research. (2000). "Race/ Ethnicity, Gender, Socioeconomic Status- Research Exploring Their Effects on Child Health: A Subject Review." Pediatrics, 105 (6), 1539- 51.

Ammerman, P. (1997). Sharing the wealth: Taking GIS data to the public, Retrieved from http://americancityandcounty.com/mag/government_sharing_wealth_taking

Andajani-Sutjahjo, S., Ball, K., Warren, N., Inglis, V., Crawford, D. (2004). Perceived personal, social and environmental barriers to weight maintenance among young women: A community survey. International Journal of Behavioral Nutrition and Physical Activity, 1, 1-15

Balfour, J., Kaplan, G. (2002). Neighborhood environment and loss of physical function in older adults: Evidence from the Alameda County Study. American Journal of Epidemiology, 155 (6), 507 - 515.

Ball, K., Bauman, A., Leslie, E., \& Owen, N. (2001). Perceived environmental aesthetics and convenience and company are associated with walking for exercise among Australian adults. Preventive Medicine, 33(5), 434-440.

Ball, K., Mishra, G., \& Crawford, D. (2002). Which aspects of socioeconomic status are related to obesity among men and women? International Journal of Obesity, 26(4), 559-565. doi: 10.1038/sj/ijo/0801960

Banks-Wallace, J., \& Conn, V. (2002). Interventions to promote physical activity among African American women. Public Health Nursing, 19(5), 321-335.

BeLue, R., Okoror, T., Iwelunmor, J., Taylor, K., Degboe, A., Agyemang, C., Ogedebe, G. (2009). An overview of cardiovascular risk factor burden in sub-Saharan African countries: A socio-cultural perspective. Global Health, 5, (10).

Binder, A., Stokols, D., Catalano, R. (1975). Social Ecology: An emerging multidiscipline. Journal of Environmental Education, 7 (2), 32- 43.

Black, J., Macinko, J. (2008). Neighborhoods and obesity. Nutrition Reviews, 66 (1), 2 20.

Blanchard, C., McGannon, K., Spence, J., Rhodes, R., Nehl, E., Baker, F., Bostwick, J. (2005). Social ecological correlates of physical activity in normal weight, overweight, and obese individuals. International Journal of Obesity (London), 29,(6), 720 - 726. 
Bleich, S. N., Thorpe, R. J., Sharif-Harris, H., Fesahazion, R., \& LaVeist, T. A. (2010). Social context explains race disparities in obesity among women. Journal of Epidemiology and Community Health, 64(5), 465-469. doi: 10.1136/jech.2009.096297

Block, J., Scribner, DeSalvo, K. (2004). Fast food, race/ethnicity, and income: A geographic analysis. American Journal of Preventive Medicine, 27, 211-217.

Bopp, M., Wilcox, S., Laken, M., Butler, K., Carter, R. E., McClorin, L., \& Yancey, A. (2006). Factors associated with physical activity among African American men and women. American Journal of Preventive Medicine, 30(4), 340-346. doi: 10.1016/j.amepre.2005.11.007

Booth, K., Pinkston, M., Poston, W. (2005). Obesity and the built environment. Journal of the American Diet Association, 105 (S1), S110 - S117.

Booth, M.L., Owen, N., Bauman, A., Clavisi, O., \& Leslie, E. (2000). Social-cognitive and perceived environmental influences associated with physical activity in older Australians. Preventive Medicine, 31(1), 15-22.

Borradaile, K., Vander, S., Sandoval, B., Karpyn, A. (2009). Snacking in children: The role of urban corner stores. Pediatrics, 12 (5), 1293 - 1298.

Boyce, C., \& Neale, P. (2006). Conducting in-depth interviews: A guide for designing and conducting in-depth interviews for evaluation input, Retrieved from http://www.cpc.unc.edu/measure/training/materials/data-qualityportuguese/m_e_tool_series_indepth_interviews.pdf

Boyington, J., et al. (2008). Cultural attitudes toward weight, diet and physical activity among overweight African American girls. Preventing Chronic Disease 5.2 (2008): 1-9. Centers for Disease Control and Prevention. Web. 24 Feb. 2013. http://www.cdc.gov/pcd/issues/2008/apr/07_0056.htm.

Brancati, F. L., Kao, W. H., Folsom, A. R., Watson, R. L., \& Szklo, M. (2000). Incident type 2 diabetes mellitus in African American and white adults: the Atherosclerosis Risk in Communities Study. JAMA, 283(17), 2253-2259.

Bronner, Y., \& Boyington, J. E. (2002). Developing weight loss interventions for African American women: elements of successful models. Journal of the National Medical Association, 94(4), 224-235.

Brown, N., Davis, S. (2003). Association of exposure to racial discrimination and socioecologic and psychosocial characteristics with hypertension status among African Americans. Annals of Epidemiology, 13, (8).

Burdette, A., Hill, T. (2008). An examination of processes linking perceived neighborhood disorder and obesity. Social Science \& Medicine, 67 (1), 38 - 46.

Burt, V. L., Whelton, P., Roccella, E. J., Brown, C., Cutler, J. A., Higgins, M., Labarthe, D. (1995). Prevalence of hypertension in the US adult population. Results from the Third National Health and Nutrition Examination Survey, 1988-1991. Hypertension, 25(3), 305-313.

Carr, D., \& Friedman, M. A. (2005). Is obesity stigmatizing? Body weight, perceived discrimination, and psychological well-being in the United States. Journal of Health and Social Behavior, 46(3), 244-259.

Centers for Disease Control and Prevention. (1996). Neighborhood safety and the prevalence of physical inactivity, Retrieved from http://www.cdc.gov/mmwr/preview/mmwrhtml/00056582.htm. 
Centers for Disease Control and Prevention. (2013). Adult obesity facts, Retrieved from http://www.cdc.gov/obesity/data/adult.html

Christian, H., Giles-Corti, B., Knuiman, M., Timperio, A., Foster, S. (2011). The influence of the built environment, social environment, and health behaviors on body mass index. Results from RESIDE. Preventive Medicine, 53, 57-60.

City of Memphis. "Community Centers" Accessed August 11, 2014. < http://www.memphistn.gov/Government/ParksNeighborhoods/CommunityCenter s.aspx $>$.

City of Memphis. Memphis \& Shelby County Community Redevelopment Agency. Accessed August 14, 2014.< http://www.memphistn.gov/boardsandcommissions/BoardMaster.aspx?ID=153>.

Cho, S., Chen, Z., Yen, S., Eastwood, D. (2006). The effects of urban sprawl on body mass index: Where people live does matter. Consumer Interests Annual, 52.

Clarke, P. J., O'Malley, P. M., Johnston, L. D., Schulenberg, J. E., \& Lantz, P. (2009). Differential trends in weight-related health behaviors among American young adults by gender, race/ethnicity, and socioeconomic status: 1984-2006. American Journal of Public Health, 99(10), 1893-1901. doi: 10.2105/ajph.2008.141317

Colditz, G. A., Willett, W. C., Rotnitzky, A., \& Manson, J. E. (1995). Weight gain as a risk factor for clinical diabetes mellitus in women. Annals of Internal Medicine, 122(7), 481-486.

Community Tool Box. "Understanding and Describing the Community". Accessed March 2014 from http://ctb.ku.edu/en/table-of-contents/assessment/assessingcommunity-needs-and-resources/describe-the-community/main.

Connor-Greene, P. A. (1988). Gender differences in body weight perception and weightloss strategies of college students. Women and Health, 14(2), 27-42. doi: 10.1300/J013v14n02_03

Consortium for Health Education Economic Empowerment and Research (CHEER). (2010). GIS maps of our community, Retrieved from http://www.uthsc.edu/CHEER/documents/CHEERLEADER_April.pdf

Cornier, M. A., Donahoo, W. T., Pereira, R., Gurevich, I., Westergren, R., Enerback, S., Draznin, B. (2005). Insulin sensitivity determines the effectiveness of dietary macronutrient composition on weight loss in obese women. Obesity Research, 13(4), 703-709. doi: 10.1038/oby.2005.79

Creswell, J., \& Plano-Clark, V. (2007). Designing and conducting mixed-methods research. Thousand Oaks, CA: Sage Publications.

Cuádraz, G. H., \& Uttal, L. (1999). Intersectionality and In-depth Interviews: Methodological Strategies for Analyzing Race, Class, and Gender. Race, Gender \& Class, 6(3), 156-186. doi: 10.2307/41674900

Dagogo-Jack, S. (2003). Ethnic disparities in type 2 diabetes: pathophysiology and implications for prevention and management. Journal of the National Medical Association, 95(9), 774, 779-789.

Daniel, K., Perrotta, K. (2009). Creating Walkable and Transit-Supportive Communities in Halton. Halton Region Health Department, Creating Walkable and TransitSupportive Communities in Halton. Oakville, Ontario: 2009.

Darity Jr, W. et al. (2010). Race, Genetics and Health: An Introduction. The Review of Black Political Economy, 37, 1-6. 
Davis, E. (2005). Racial and Socioeconomic Differences in the Weight- Loss Experiences of Obese Women. American Journal of Public Health, 95, 1539- 1543.

Davis, E. M., Rovi, S., \& Johnson, M. S. (2005). Mental health, family function and obesity in African American women. Journal of the National Medical Association, 97(4), 478-482.

Denzin, N. K., \& Lincoln, Y. S. (2008). The landscape of qualitative research. Thousand Oaks, CA: Sage Publishing.

Department of Agriculture. (2014). Retail food store inspections. Accessed August 14, 2014. http://www.tn.gov/agriculture/regulatory/foodstores.shtml

Diabetes: Successes and opportunities for population- based prevention and control: at a glance 2011. Atlanta, GA: Centers for Disease Control and Prevention; 2011, Aug 1. Available from: www.cdc.gov/chronicdisease/resources/publications/AAG/ddt.htm.

Diabetes in African Americans. "(2002). National Institute of Diabetes and Digestive and Kidney Diseases. NIH Publication. Bethesda (MD): US Department of Health and Human Services, National Institutes of Health. 02-3266

"The Diabetes Epidemic in African Americans." The National Institute of Diabetes and Digestive and Kidney Diseases. 2008. National Diabetes Information Clearinghouse. Web. 25 Oct. 2009. <http://www.diabetes.niddk.nih.gov/dm/pubs/statistics/>

Diabetes Prevention Program Research Group. (2002). Reduction in the Incidence of type 2 diabetes with lifestyle intervention or Metformin. The New England Journal of Medicine 346.6 393-403.

Dixon, J. B. (2010). The effect of obesity on health outcomes. Molecular and Cellular Endocrinology, 316(2), 104-108. doi: 10.1016/j.mce.2009.07.008

Dodani, S., \& Fields, J. Z. (2010). Implementation of the fit body and soul, a churchbased life style program for diabetes prevention in high-risk African Americans: a feasibility study. Diabetes Educator, 36(3), 465-472. doi: 10.1177/0145721710366756

Douglas, J. G., Bakris, G. L., Epstein, M., Ferdinand, K. C., Ferrario, C., Flack, J. M., . . . Vidt, D. G. (2003). Management of high blood pressure in African Americans: consensus statement of the Hypertension in African Americans Working Group of the International Society on Hypertension in Blacks. Archives of Internal Medicine, 163(5), 525-541.

Downtown Memphis Commission."About us" Accessed August 12 2014. < http://www.downtownmemphiscommission.com/about.html>.

Doyle, S., Kelly-Schwartz, A., Schlossberg, M., Stockard, J. (2006). Active community environments and health: The relationship of walkable and safe communities to individual health. Journal of the American Planning Association, 72 (1). 19 - 31.

Drewnowski, A., \& Specter, S. E. (2004). Poverty and obesity: the role of energy density and energy costs. American Journal of Clinical Nutrition, 79(1), 6-16.

Duncan, D. T., Wolin, K. Y., Scharoun-Lee, M., Ding, E. L., Warner, E. T., \& Bennett, G. G. (2011). Does perception equal reality? Weight misperception in relation to weight-related attitudes and behaviors among overweight and obese US adults. Int $J$ Behav Nutr Phys Act, 8, 20. doi: 10.1186/1479-5868-8-20

Egede, L., Bonadonna, R. (2003). diabetes self-management in African Americans: An exploration of the role of fatalism. The Diabetes Educator, 29 (1), 105 - 115. 
Egede, L. E., \& Dagogo-Jack, S. (2005). Epidemiology of type 2 diabetes: focus on ethnic minorities. Medical Clinics of North America, 89(5), 949-975, v2i. doi: 10.1016/j.mcna.2005.03.004

Elwood, S., \& Leitner, H. (1998). GIS and Community-based Planning: Exploring the Diversity of Neighborhood Perspectives and Needs. Cartography and Geographic Information Systems, 25(2), 77-88. doi: 10.1559/152304098782594553

Ewing, R., Meakins, G., Hamidi, S., Nelson, A. (2014). Relationship between urban sprawl and physical activity, obesity, and morbidity - Update and refinement. Health \& Place, 26, $118-126$.

Everett, M. (2011). They say it runs in the family: diabetes and inheritenace in Oaxaca, Mexico. Social Science \& Medicine, 72 (11), 1776 - 1783.

Eyler, A., Maison-Koffman, D., Young, D. et al. (2003). Quantitative study of correlates of physical activity in women from diverse racial/ethnic groups. American Journal of Preventive Medicine, 25(suppl 1), 5 - 36.

Faith, M. S., Flint, J., Fairburn, C. G., Goodwin, G. M., \& Allison, D. B. (2001). Gender differences in the relationship between personality dimensions and relative body weight. Obesity Research, 9(10), 647-650. doi: 10.1038/oby.2001.86

Farrell, M. H., Murphy, M. A., \& Schneider, C. E. (2002). How underlying patient beliefs can affect physician-patient communication about prostate-specific antigen testing. Effective Clinical Practice, 5(3), 120-129.

The Federal Bureau of Investigation. (2010) Crime Statistics for 38106, 38109, Tennessee, and Shelby County. Accessed April 24, 2014.

$<$ http://www.fbi.gov/stats-services/crimestats.

Feng, J., Glass, T., Curriero, F., Stewart, W., Schwartz, B. (2010). The built environment and obesity: a systematic review of the epidemiologic evidence. Health \& Place, 16, $175-190$.

Finkelstein, E. A., Fiebelkorn, I. C., \& Wang, G. (2003). National medical spending attributable to overweight and obesity: how much, and who's paying? Health Affairs, Suppl Web Exclusives, W3-219-226.

Finkelstein, E. A., Fiebelkorn, I. C., \& Wang, G. (2004). State-level estimates of annual medical expenditures attributable to obesity. Obesity Research, 12(1), 18-24. doi: 10.1038/oby.2004.4

"Food security in the United States, Committee Report". House Select Committee on Hunger Washington, DC: U.S. Government Printing Office, October 1990.

Ford, E. S., Giles, W. H., \& Dietz, W. H. (2002). Prevalence of the metabolic syndrome among US adults: findings from the third National Health and Nutrition Examination Survey. JAMA, 287(3), 356-359.

Frank, L., Sallis, J., Conway, T., Chapman, J., Saelens, B., \& Bachman, W. (2006). Many pathways from land use to health: Associations between neighborhood walkability and active transportation, body mass index, and air quality. Journal of the American Planning Association, 72 (1), 75 - 87.

Frongillo, E. A. (2003). Understanding obesity and program participation in the context of poverty and food insecurity. Journal of Nutrition, 133(7), 2117-2118. 
Gans, K. M., Kumanyika, S. K., Lovell, H. J., Risica, P. M., Goldman, R., Odoms-

Gary, T., Safford, M., Gerzoff, R., Ettner, S., Karter, A., Beckles, G., Brown, A. (2008).

Perception of neighborhood problems, health behaviors, and diabetes outcomes among adults with diabetes in managed care. Diabetes Care, 37 (4), 273-278.

Green, J., \& Thorogood, N. (2004). Qualitative methods for health research (4th ed.). Great Britain: Sage Publications.

Gorin, A., Phelan, S., Tate, D., Sherwood, N., Jeffrey, R., Wing, R. (2005). Involving support partners in obesity treatment. Journal of Consulting and Clinical Psychology, 73(2), 341 - 343.

Harrington, D., Elliott, S. (2009). Weighing the importance of neighborhood: A multilevel exploration of the determinant of overweight and obesity. Social Science \& Medicine, 68 (4), 593 - 600.

Harrison, T., Hindorff, L., Kim, H., Wines, R., Bowen, D., McGrath, B., Edwards, K. (2003). Family history of diabetes as a potential public health tool. American Journal of Preventive Medicine, 24 (2), 162 - 169.

Havas, S, Treiman, K., Langenburg, P., Ballesteros, M., Anliker, J., Damron, D., Feldman, R. (1998). Factors associated with fruit and vegetable consumption among women participating in WIC. Journal of the American Dietary Association, 98, 1141 - 1148.

Hedley, A. A., Ogden, C. L., Johnson, C. L., Carroll, M. D., Curtin, L. R., \& Flegal, K. M. (2004). Prevalence of overweight and obesity among US children, adolescents, and adults, 1999-2002. JAMA, 291(23), 2847-2850. doi: 10.1001/jama.291.23.2847

Hill, J., Nielsen, M., Fox, M. (2013). Understanding the social factors that contribute to diabetes: A means to informing health care and social policies for the chronically ill. The Permenente Journal, 17 (2) 67 - 72.

Hoefer, R. A., Hoefer, R. M., \& Tobias, R. A. (1994). Geographic information systems and human services. Journal of community Practice, 1(13), 113-127.

Hosler, A., et al. (2006). Low-Fat Milk and High-Fiber Bread Availability in Food Stores in Urban and Rural Communities. Journal of Public Health Management Practice, 12, 556-562.

Hu, H., Cho, J., Huang, G., Wen, F., Choi, S., Shih, M., Lightstone, A. (2014). Neighborhood environment and health behavior in Los Angeles area. Transport Policy, 33, $40-47$.

Humpel, N., Owen, N., Leslie, E. (2002). Environmental factors associated with adults' participation in physical activity: a review. American Journal of Preventive Medicine, 22(3), 199-199.

Hutchison, P., Bodor, J., Swalm, C., Rice, J., Rose, D. (2012). Neighborhood food environments and obesity in southeast Louisiana. Health \& Place, 18 (4), 854 860.

James, P., Troped, P., Hart, J., Joshu, C., Colditz, G., Brownson, R., Ewing, R., Laden, F. (2013). Urban sprawl, physical activity, and body mass index: nurses' health study and nurses' health study. American Journal of Public Health, 103, (2), 369 - 375.

Jeffery, R. W., Drewnowski, A., Epstein, L. H., Stunkard, A. J., Wilson, G. T., Wing, R. R., \& Hill, D. R. (2000). Long-term maintenance of weight loss: current status. Health Psychology, 19(1 Suppl), 5-16. 
Jeffrey R., Forster J., Folsom, A., Luepker, R., Jacobs, D., Blackburn, H. (1989). The relationship between social status and body mass index in the Minnesota Heart Health Program. International Journal of Obesity, 13, 59 - 67.

Jenkins, C., McNary, S., Carlson, B. A., King, M. G., Hossler, C. L., Magwood, G., . . . Ma'at, I. (2004). Reducing disparities for African Americans with diabetes: progress made by the REACH 2010 Charleston and Georgetown Diabetes Coalition. Public Health Reports, $119(3), 322-330$. doi:

10.1016/j.phr.2004.04.011

Johnston, O., Reilly, J., \& Kremer, J. (2004). Women's experiences of appearance concern and body control across the lifespan: challenging accepted wisdom. Journal of Health Psychology, 9(3), 397-410. doi: 10.1177/1359105304042349

Joshua, C.E., Boehmer, T., Brownson, R., Ewing, R. (2008). Personal, neighborhood and urban factors associated with obesity in the United States. Journal of Epidemiology and Community Health, 62, 202 - 208.

Karnehed, N., Rasmussen, F., \& Kark, M. (2007). Obesity in young adulthood and later disability pension: a population-based cohort study of 366,929 Swedish men. Scand J Public Health, 35(1), 48-54. doi: 10.1080/14034940600858524

Kelly-Schwartz, A., Stockard, J., Doyle, S., Sclossberg, M. (2004). Is sprawl unhealthy? A multilevel analysis of the relationship of metropolitan sprawl to the health of individuals. Journal of Planning Education and Research, 24, 184 - 196.

Kim, D., Subraminian, S., Gortmaker, S., Kawachi, I. (2006). US State and county level social capital in relation to obesity and physical inactivity: A multilevel multivariable analysis. Social Science \& Medicine, 63 (4), 1045 - 1059.

Kimm S., et al. (2001). Racial divergence in adiposity during adolescence: the NHLBI Growth and Health Study. Pediatrics, 107, E34.

King, A., Castro, C., Wilcox, S., Eyler, A., Sallis, J., \& Brownson, R. (2000). Personal and environmental factors associated with physical inactivity among different racial-ethnic groups of U.S. middle-aged and older-aged women. Health Psychology, 19(4), 354-364.

King, A., Sallis, J., Frank, L., Saelens, B., Cain, K., Conway, T., Chapman, J., Ahn, D., Kerr, J. (2011). Aging in neighborhoods differing in walkability and income: Associations with physical activity and obesity in older adults. Social Science \& Medicine, 73 (10), 1525 - 1533.

Knowler, W. C., Barrett-Connor, E., Fowler, S. E., Hamman, R. F., Lachin, J. M., Walker, E. A., \& Nathan, D. M. (2002). Reduction in the incidence of type 2 diabetes with lifestyle intervention or metformin. New England Journal of Medicine, 346(6), 393-403. doi: 10.1056/NEJMoa012512

Kostova, D. (2011). Can the built environment reduce obesity quest; the impact of residential sprawl and neighborhood parks on obesity and physical activity. Eastern Economic Journal, 37 (3), 390 - 402.

Kronenfeld, L., et al. (2010). Ethnic and racial differences in body size perception and satisfaction. Body Image, 6, 1-6.

Kumanyika, S., et al. (1993). Special issues regarding obesity in minority communities." Annals of Internal Medicine, 119, (7), 650-654.

Kumunuika, S., et al. (1996). Cultural aspects of African American eating patterns. Ethnicity \& Health, 1 (3). 
Layton, K., Parker, S., Hermann, J., Williams, S. (2009). Comparison of socioecologic factors affecting overweight and obesity among limited income African American, Native American, and White women. Journal of Nutrition Education and Behavior, 41, (4, SJuly-SAugust) S43.

Lasco RA et al. (1989). Participation rates, weight loss, and blood pressure changes among obese women in a nutrition-exercise program. Public Health Rep, 104, 640-646.

Lean, M. E., Powrie, J. K., Anderson, A. S., \& Garthwaite, P. H. (1990). Obesity, weight loss and prognosis in type 2 diabetes. Diabetic Medicine, 7(3), 228-233.

Lee, I., Ewing, R., Sesso, H. (2009). The built environment and physical activity levels: The Harvard Alumni health study. American Journal of Preventive Medicine, 37 (4), 293 - 298.

Lewis L.B., et al. (2005). African Americans' Access to Healthy Food Options in South Los Angeles Restaurants. American Journal of Public Health. 95, 668- 675.

Lincoln, Y., Guba, E. (1985). Naturalistic inquiry. (1 ${ }^{\text {st }}$ Ed.) SAGE Publications.

Lopez-Azpiazu I., Martinez-Gonzales, M., Kearney, J., Gibney, M., Martinez, A. (1999). Perceived barriers of, and benefits to, healthy eating reportedd by Spanish national sample. Public Health Nutrition, 2, 209 - 205.

Mama, S. K., Quill, B. E., Fernandez-Esquer, M. E., Reese-Smith, J. Y., Banda, J. A., \& Lee, R. E. (2011). Body image and physical activity among Latina and African American women. Ethnicity and Disease, 21(3), 281-287.

Marshall, M. (2005). diabetes in African Americans. Postgraduate Medical Journal, 81, $734-740$.

McMurray, R., Harrel, J., Deng, S., Bradley, C., Cox, I., Bangdiwala, S. (2000). The influence of physical activity, socioeconomic status, and ethnicity on the weight status of adolescents. Obesity Research, 8, 130 - 139.

McLaren, L. (2007). Socioeconomic status and obesity. Epidemiologic Reviews, 29, 2948. doi: 10.1093/epirev/mxm001

Melnyk, M., Weinstein, E. (1994). Preventing obesity in black women by targeting adolescents: a literature review." Journal of the American Dietetic Association, 94 (5), 536.

Memphis and Shelby County Health Department. "Rabies Control". Accessed October 11, 2014. < http://www.shelbycountytn.gov/DocumentCenter/Home/View/751>

Michael, Y., Nagel, C., Gold, R., Hillier, T. (2014). Does change in the neighborhood environment prevent obesity in older women? Social Science \& Medicine, 102, $129-137$.

Mobley, L. R., Root, E. D., Finkelstein, E. A., Khavjou, O., Farris, R. P., \& Will, J. C. (2006). Environment, obesity, and cardiovascular disease risk in low-income women. American Journal of Preventive Medicine, 30(4), 327-332. doi: 10.1016/j.amepre.2005.12.001

Morland K., Roux, A.V., Wing, S. (2006). Supermarkets, other food stores and obesity: The atherosclerosis risk in communities study. American Journal of Preventative Medicine, 30, 333-339.

Must, A., Spadano, J., Coakley, E. H., Field, A. E., Colditz, G., \& Dietz, W. H. (1999). The disease burden associated with overweight and obesity. JAMA, 282(16), 1523-1529. 
Muth, J. L., \& Cash, T. F. (1997). Body-Image Attitudes: What Difference Does Gender Make?1. Journal of Applied Social Psychology, 27(16), 1438-1452. doi:

10.1111/j.1559-1816.1997.tb01607.x

National Diabetes Statistics, 2011. Bethesda, MS: US Department of Health and Human Services: National Institutes of Health; 2012 Feb. Available from: http://diabetes.niddk.nih.gov/dm/pubs/statistics.

Nguyen, D. (2010). Evidence of the impacts of urban sprawl on social capital. Environmental Planning B: Planning and Design. 37 (4), 610 - 627.

Northridge, M., Sclar, E., Biswas, P. (2003). Sorting out the connections between the built environment and health: a conceptual framework for navigating pathways and planning healthy cities. Journal of Urban Health, 80, 556 - 568.

Ogden C. L., Carroll, M. D., Kit, B.K., \& Flegal K. M. (2014). Prevalence of childhood and adult obesity in the United States, 2011-2012. Journal of the American Medical Association, 311(8), 806-814.

Pan, X. R., Li, G. W., Hu, Y. H., Wang, J. X., Yang, W. Y., An, Z. X., . . Howard, B. V. (1997). Effects of diet and exercise in preventing NIDDM in people with impaired glucose tolerance. The Da Qing IGT and Diabetes Study. Diabetes Care, 20(4), 537-544.

Papas, M. A., Alberg, A. J., Ewing, R., Helzlsouer, K. J., Gary, T. L., \& Klassen, A. C. (2007). The built environment and obesity. Epidemiologic Reviews, 29, 129-143. doi: 10.1093/epirev/mxm009

Partners in Action. (n/a). Appendix A: Models, factors and theories of change, Retrieved from http://depts.washington.edu/waaction/plan/append/a.html

Pereira, M. A., Jacobs, D. R., Jr., Van Horn, L., Slattery, M. L., Kartashov, A. I., \& Ludwig, D. S. (2002). Dairy consumption, obesity, and the insulin resistance syndrome in young adults: the CARDIA Study. JAMA, 287(16), 2081-2089.

Perez, D., Ang, A., Vega, W. (2009). Effects of health insurance on perceived quality of care among latinos in the United States. Journal of General Internal Medicine, 24 (Suppl 3), 555-560.

Pi-Sunyer, F. X. (2005). Weight loss in type 2 diabetic patients. Diabetes Care, 28, 15261527.

Pi-Sunyer, X., Blackburn, G., Brancati, F. L., Bray, G. A., Bright, R., Clark, J. M., . . . Yanovski, S. Z. (2007). Reduction in weight and cardiovascular disease risk factors in individuals with type 2 diabetes: one-year results of the look AHEAD trial. Diabetes Care, 30(6), 1374-1383. doi: 10.2337/dc07-0048

Platinga, A., Bernell, S. (2007). The association between urban sprawl and obesity: is it a two way street? Journal of Regional Science, 47 (5), 857 - 879.

Pliner, P., Chaiken, S., \& Flett, G. L. (1990). Gender Differences in Concern with Body Weight and Physical Appearance Over the Life Span. Personality and Social Psychology Bulletin, 16(2), 263-273. doi: 10.1177/0146167290162007

Poortinga, W. (2014). Neighborhood environment, physical activity, and obesity. Reference modeule in Earth systems and environmental sciences. Encyclopedia of Environmental Health, 2011, 44 - 53.

Powell, L., et al. (2007). The Availability of Fast-Food and Full-Service Restaurants in the United States: Associations with Neighborhood characteristics. American Journal of Preventative Medicine. 33, S240-S245. 
Prochaska, J. O., \& Velicer, W. F. (1997). The transtheoretical model of health behavior change. American Journal of Health Promotion, 12(1), 38-48.

Rabjee, F. (2004). Focus group interview and data anlysis. Proceedings of the Nutrition Society, 63, 655-660.

Racial and Ethnic Approaches to Community Health (REACH). Atlanta, GA: Centers for Disease Control and Prevention; 2012 Nov 6. Available from www.cdc.gov/reach/about.htm.

Rana, J. S., Li, T. Y., Manson, J. E., \& Hu, F. B. (2007). Adiposity compared with physical inactivity and risk of type 2 diabetes in women. Diabetes Care, 30(1), 53-58. doi: 10.2337/dc06-1456

Rattan, A., Campese, A., \& Eden, C. (2012). Modeling walkability. Retrieved from http://www.esri.com/news/arcuser/0112/files/walkability.pdf.

Reidpath, D., Burns, C., Carrard, J., Mahoney, M., Townsend, M. (2002). An ecological study of the relationship between social and environmental determinants of obesity. Health Place, 8, $141-145$.

Renders, C., Gerlof, V., Griffin, S., Wagner, D., Eijk van, J.., Assendelft, W. (2001). Interventions to improve the management of diabetes in primary care, outpatient, and community settings A systematic review. Diabetes Care, 24 (10), 1821 1833

Ritchie, J., \& Spencer, L. (1994). Qualititave data analysis for applied policy research. In A. Bryman \& R. G. Burgess (Eds.), Analysing Qualitative Data (pp. 173-194). London: Routledge.

Rowe, J. (2010). Voices from the inside: African American women's perspectives on healthy lifestyles. Health Education and Behavior, 37(6), 789-800. doi: $10.1177 / 1090198110365992$

Sallis, J.F., Johnson, M.F., Calfas, K.J., Caparosa, S., \& Nichols J.F. (1997). Assessing perceived physical environmental variables that may influence physical activity. Research quarterly for Exercise and Sport, 68(4), 345-351.

Samuel-Hodge, C., Headen, S., Skelly, A., Ingram, A., Keyserling, T., Jackson, E., Ammerman, A., Elasy, T. (2000). Influences on day-to-day self-management of type 2 diabetes among African American women: spirituality, the multi-caregiver role, and other social context factors. Diabetes Care, 23 (7), 928 - 933.

Sanderson, B., Littleton, M., \& Pulley, L. (2002). Environmental, policy, and cultural factors related to physical activity among rural, African American women. Women and Health, 36(2), 75-90.

Sarma, S., Zaric, G., Campbell, M., Gilliand, J. (2014). The effect of physical activity on adult obesity: Evidence from the Canadian NPHS Panel. Economics and Human Biology, In Press.

Save-a-lot Food Stores. "About Save-a-Lot Stores \& Discount Groceries" Accessed August 11, 2014. < http://save-a-lot.com/about-save-a-lot>.

Schutchfield, F., Howard, A. (2011). Moving on upstream: The rold of health departments in addressing socioecologic determinants of disease. American Journal of Preventive Medicine, 40 (S1), S80 - S83.

Seidell, J. C. (1995a). The impact of obesity on health status: some implications for health care costs. International Journal of Obesity and Related Metabolic Disorders, 19 Suppl 6, S13-16. 
Seidell, J. C. (1995b). Obesity in Europe: scaling an epidemic. International Journal of Obesity and Related Metabolic Disorders, 19 Suppl 3, S1-4.

Silverman, D. (2008). Interpreting qualitative data. Thousand Oaks, CA: Sage Publications.

Sivalingam, S. K., Ashraf, J., Vallurupalli, N., Friderici, J., Cook, J., \& Rothberg, M. B. (2011). Ethnic differences in the self-recognition of obesity and obesity-related comorbidities: a cross-sectional analysis. Journal of General Internal Medicine, 26(6), 616-620. doi: 10.1007/s11606-010-1623-3

Sloane, D, et al. (2006). Assessing Resource Environments to Target Prevention Interventions in Community Chronic Disease Control. Journal of Health Care for the Poor and Underserved, 17, 146-158.

Smith, C. (2012). Living with sugar: Influence of cultural beliefs on type 2 diabetes selfmanagement of English-speaking Afro-Caribbean women. Journal of Immigrant and Minority Health, 14 (4), 640 - 647.

Stephens, S., Cobiac, L., Veerman, J. (2014). Improving diet and physical activity to reduce population prevalence of overweight and obesity: An overview of current evidence. Preventive Medicine, 62, 167 - 178.

Stevens, J., Kumanyika, S., Keil, J. (1994). Attitudes toward body size and dieting: Difference between elderly black and whites. American Journal of Public Health. 84, $1322-1325$.

Stevens, J. (2000). Obesity and mortality in African Americans. Nutrition Reviews, 58(11), 346-353.

Stoecker, R. (2008). The CDC model of urban redevelopment: A critique and an alternative. Journal of Urban Affairs, 19 (1), 1-22.

Stokols, D. (1996). Translating social ecological theory into guidelines for community health promotion. American Journal of Health Promotion, 10 (4), 282 - 298.

Strauss, A., \& Corbin, J. (1990). Basics of qualitative research: Grounded theory procedures and techniques. Newbury Park, CA: Sage Publications.

Strauss, A., \& Corbin, J. (1998). Basics of qualitative research: Techniques and procedures for developing grounded theory. Thousand Oaks, CA: Sage Publications.

Sturm, R., Cohen, D. (2004). Suburban sprawl and physical and mental health. Public Health, 118 (7), 488 - 496.

Sullivan, S., Brashear, M., Broyles, S., Rung, A. (2014). Neighborhood environments and obesity among Afro-Carribean, African American, and Non-Hisptanic white adults in the United States: Results from the National Survey of American Life. Preventive Medicine, 61, 1-5.

Sundquist, J., Johansson, S. (1998). The influence of socioeconomic status, ethnicity, and lifestyle on body mass index in a longitudinal study. International Journal of Epidemology, 27, 57- 63.

Talen, E., \& Shah, S. (2007). Neighborhood Evaluation Using GIS: An Exploratory Study. Environment and Behavior, 39(5), 583-615. doi: 10.1177/0013916506292332

Taylor, W. C., Baranowski, T., \& Young, D. R. (1998). Physical activity interventions in low-income, ethnic minority, and populations with disability. American Journal of Preventive Medicine, 15(4), 334-343. 
Teitz, M., \& Chapple, K. (1998). The causes of inner-city poverty: Eight hypotheses in search of reality. A Journal of Development and Research, 3 (3), 33 - 69.

The Works. (2014). About The Works. Accessed August 14, 2014 from $<$ http://theworkscdc.org/about-us/>.

Thompson, D., \& Wolf, A. M. (2001). The medical-care cost burden of obesity. Obesity Reviews, 2(3), 189-197.

Tresidder, M. (2005). Using GIS to measure connectivity: An exploration of issues, Retrieved from http://web.pdx.edu/ jdill/Tresidder_Using_GIS_to_Measure_Connectivity.pdf

Tuomilehto, J., Lindstrom, J., Eriksson, J. G., Valle, T. T., Hamalainen, H., IlanneParikka, P., . . Uusitupa, M. (2001). Prevention of type 2 diabetes mellitus by changes in lifestyle among subjects with impaired glucose tolerance. New England Journal of Medicine, 344(18), 1343-1350. doi: 10.1056/nejm200105033441801

University of Memphis, Methodist Le Bonheur Center for Healthcare Economics. (2012). Potentially Avoidable Hospitalizations in Tennessee, 2009. Retrieved from University of Memphis website: http://www.memphis.edu/mlche/pdfs/hospitalizations/issuebriefpahsinshelbytn20 09may042012.pdf

US Census Bureau; "American FactFinder". Generated by Adole Muruako; using American Factfinder; http://factfinder2.census.gov; (10 February 2014).

US Department of Housing and Urban Development. (2014). Memphis Urban Renewal Community. Accessed August 14 2014. < http://portal.hud.gov/hudportal/documents/huddoc?id=19464_appsummary.pdf $>$

Urban grocery gap, Committee Report. House Select Committee on Hunger. Washington, DC: U.S. Government Printing Office, October 1992.

Wang, Y., Beydoun, M. A., Liang, L., Caballero, B., \& Kumanyika, S. K. (2008). Will all Americans become overweight or obese? estimating the progression and cost of the US obesity epidemic. Obesity (Silver Spring), 16(10), 2323-2330. doi: 10.1038/oby.2008.351

Welk, G. (2002). Physical activity assessments for health-related research. Champaign, Ill: Human Kinetics.

Whitaker, R. C., Wright, J. A., Pepe, M. S., Seidel, K. D., \& Dietz, W. H. (1997). Predicting Obesity in Young Adulthood from Childhood and Parental Obesity. New England Journal of Medicine, 337(13), 869-873. doi: doi:10.1056/NEJM199709253371301

Wilbur, J., Michaels Miller, A., Chandler, P., \& McDevitt, J. (2003). Determinants of physical activity and adherence to a 24 -week home-based walking program in African American and Caucasian women. Research in Nursing and Health, 26(3), 213-224. doi: 10.1002/nur.10083

Wilcox, S., Bopp, M., Oberrecht, L., Kammermann, S. K., \& McElmurray, C. T. (2003). Psychosocial and perceived environmental correlates of physical activity in rural and older African American and white women. Journals of Gerontology. Series B: Psychological Sciences and Social Sciences, 58(6), P329-337.

Wilson, D.B., Sargent, R.G., Dias J. (1994). Racial differences in selection of ideal body size by adolescent females. Obesity Research. 2, 38-43. 
Wolf A., et al. (1993). Activity, inactivity, and obesity: racial, ethnic, and age differences among schoolgirls. American Journal of Public Health, 83, 1625-7.

Wolfe, W. A. (2004). A review: maximizing social support--a neglected strategy for improving weight management with African American women. Ethnicity and Disease, 14(2), 212-218.

Young, A., Lasater, T. M. (2003). The development of SisterTalk: a cable TV-delivered weight control program for black women. Preventive Medicine, 37(6 Pt 1), 654667.

Young, D. R., Gittelsohn, J., Charleston, J., Felix-Aaron, K., \& Appel, L. J. (2001). Motivations for exercise and weight loss among African American women: focus group results and their contribution towards program development. Ethnicity and Health, 6(3-4), 227-245. doi: 10.1080/13557850120078143

Young, D. R., \& Stewart, K. J. (2006). A church-based physical activity intervention for African American women. Family and Community Health, 29(2), 103-117.

Zenk S.N., et al. (2005). Fruit and vegetable intake in African Americans income and store characteristics. American Journal of Preventative Medicine. 29, 1-9.

Zhang, Q., Wang, Y., \& Huang, E.S. (2009). Changes in racial/ethnic disparities in the prevalence of type 2 diabetes by obesity level among U.S. adults. Ethnicity \& Health, 14(5), 439-457.

Zhang, X., Holt, J., Lu, H., Onufrak, S., Yang, J., French, S., Sui, D. (2014). Neighborhood commuting environment and obesity in the United States: An urban-rural stratified multilevel analysis. Preventive Medicine, 59, 31-36. 
APPENDIX A. PHOTOGRAPHS OF 38106 AND 38109 EXHIBITING POTENTIAL BARRIERS TO PHYSICAL ACTIVITY
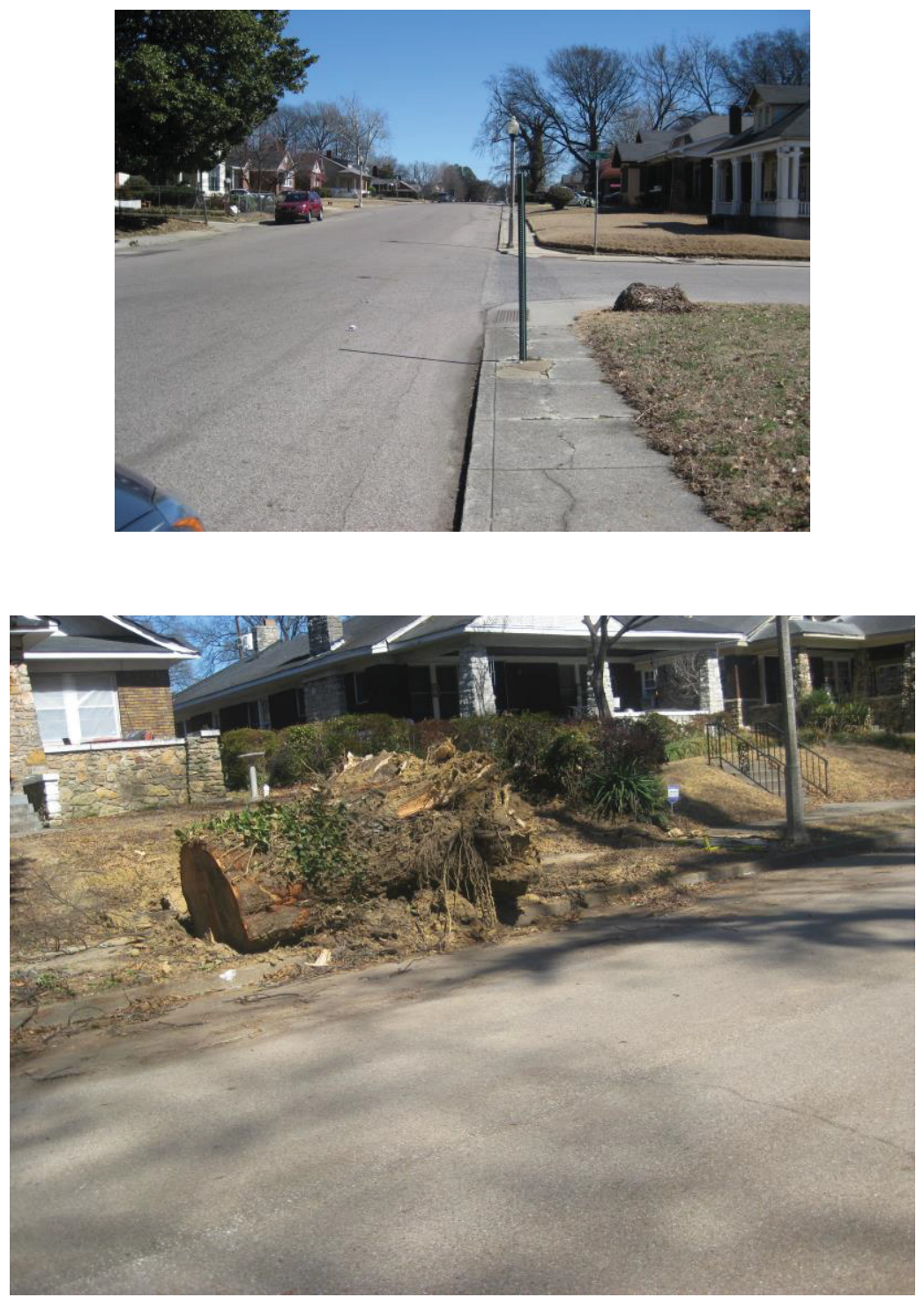

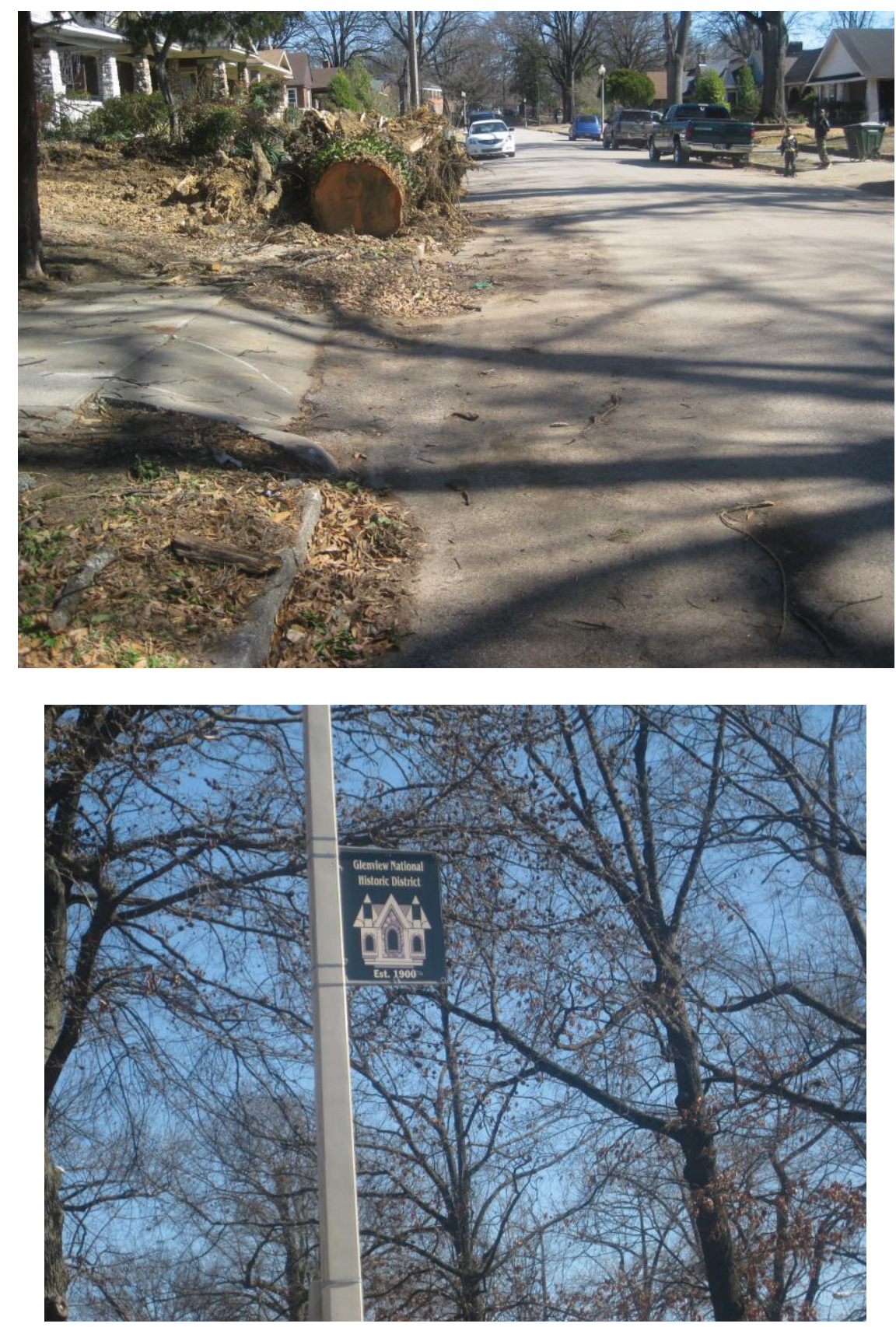

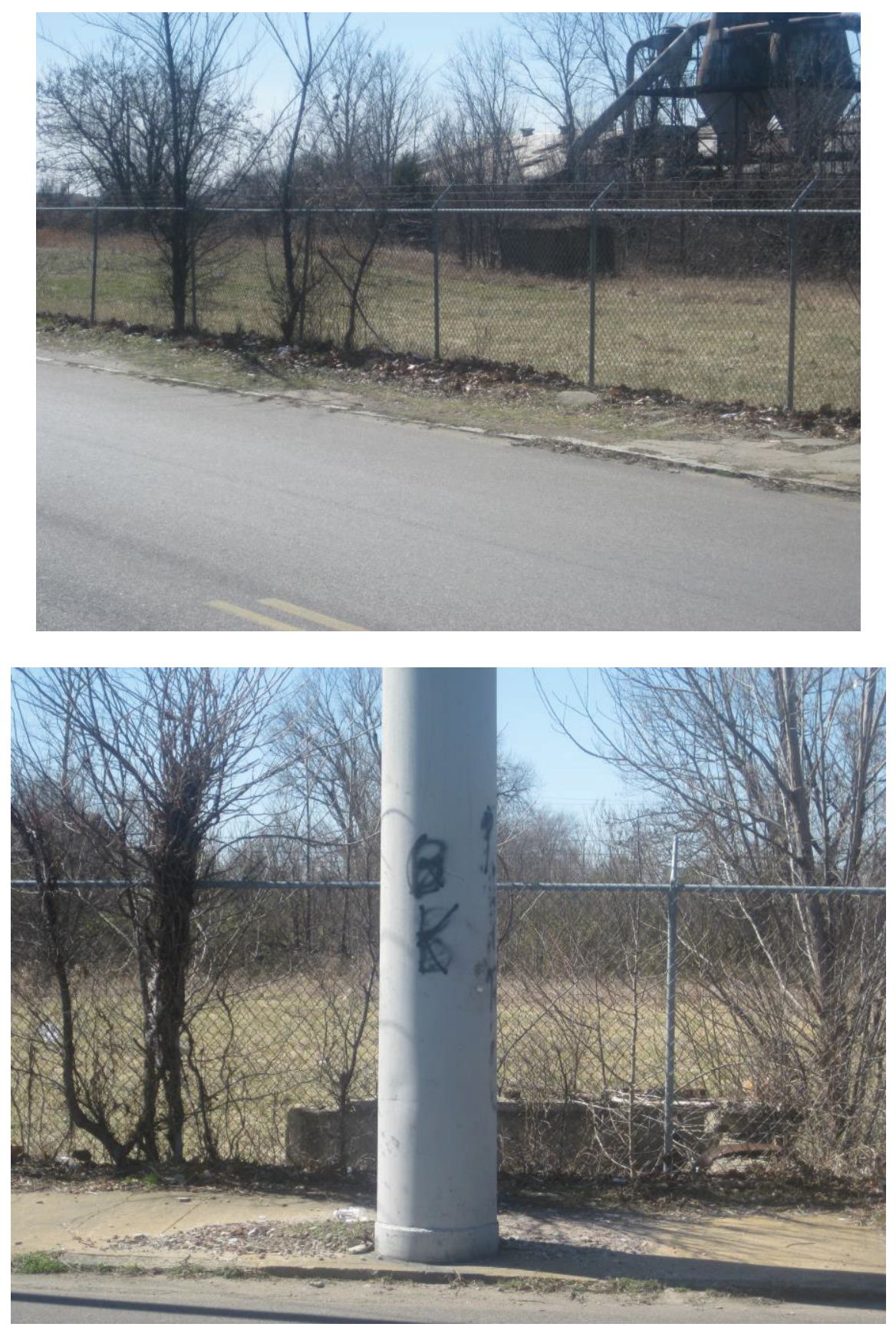

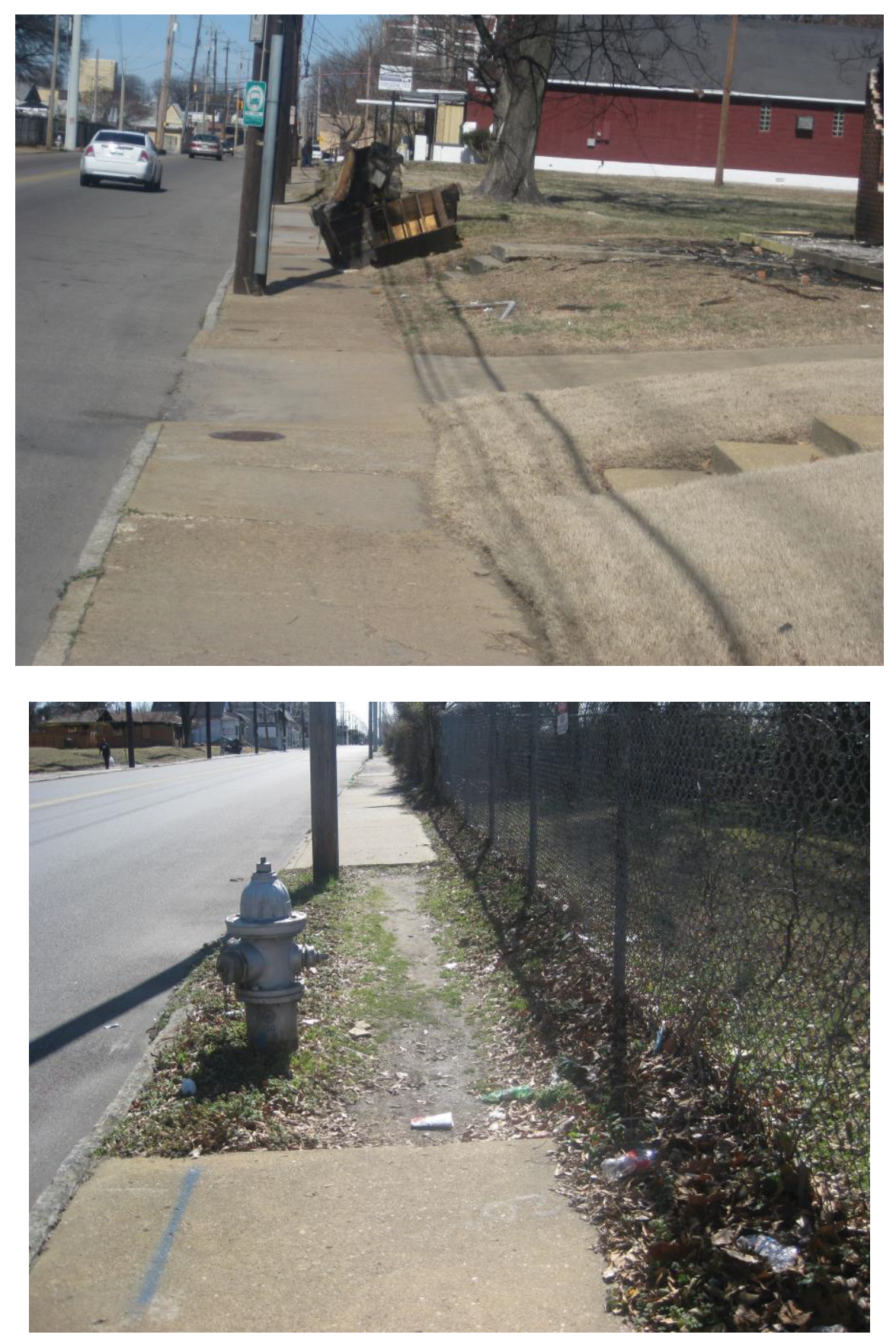

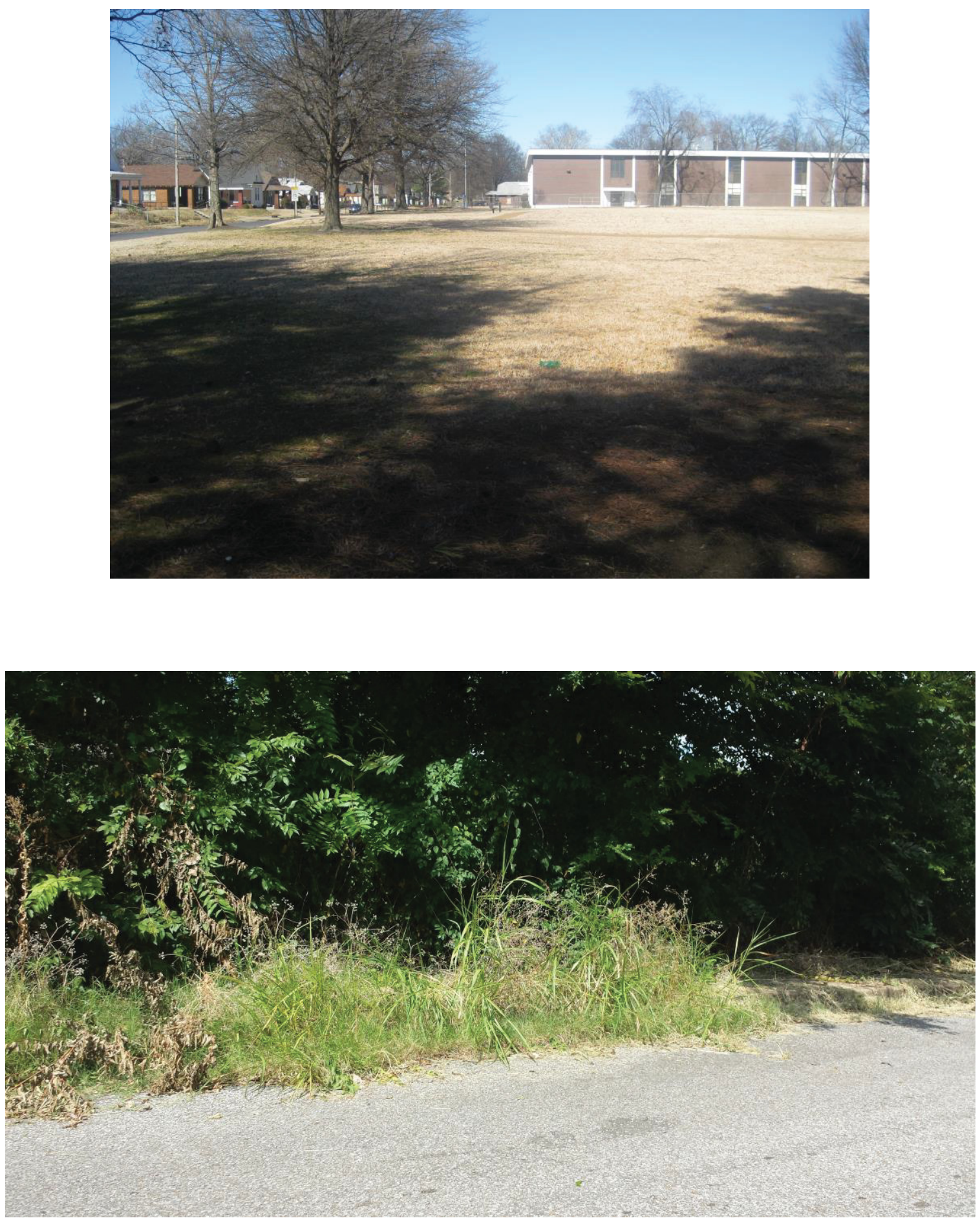

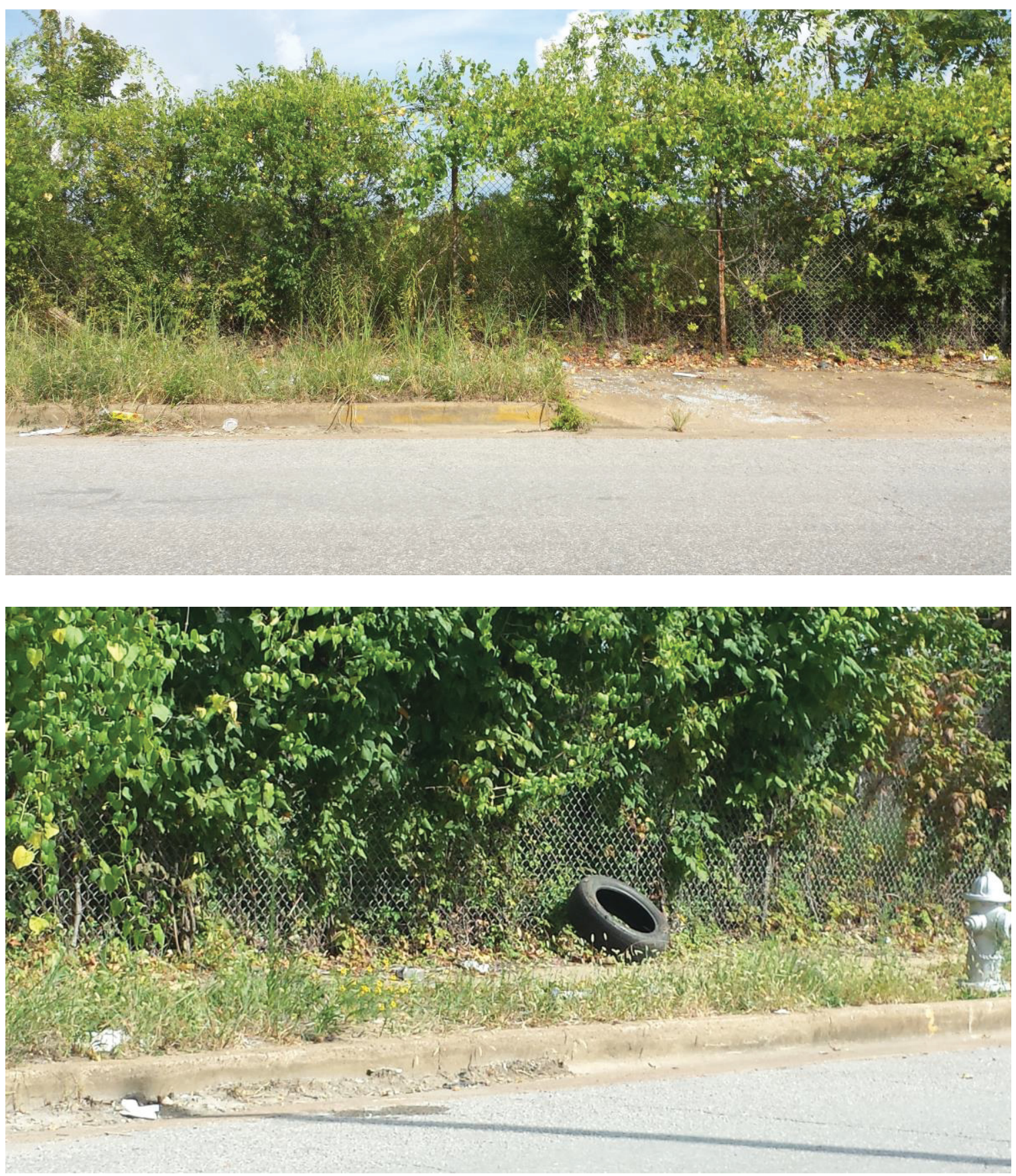


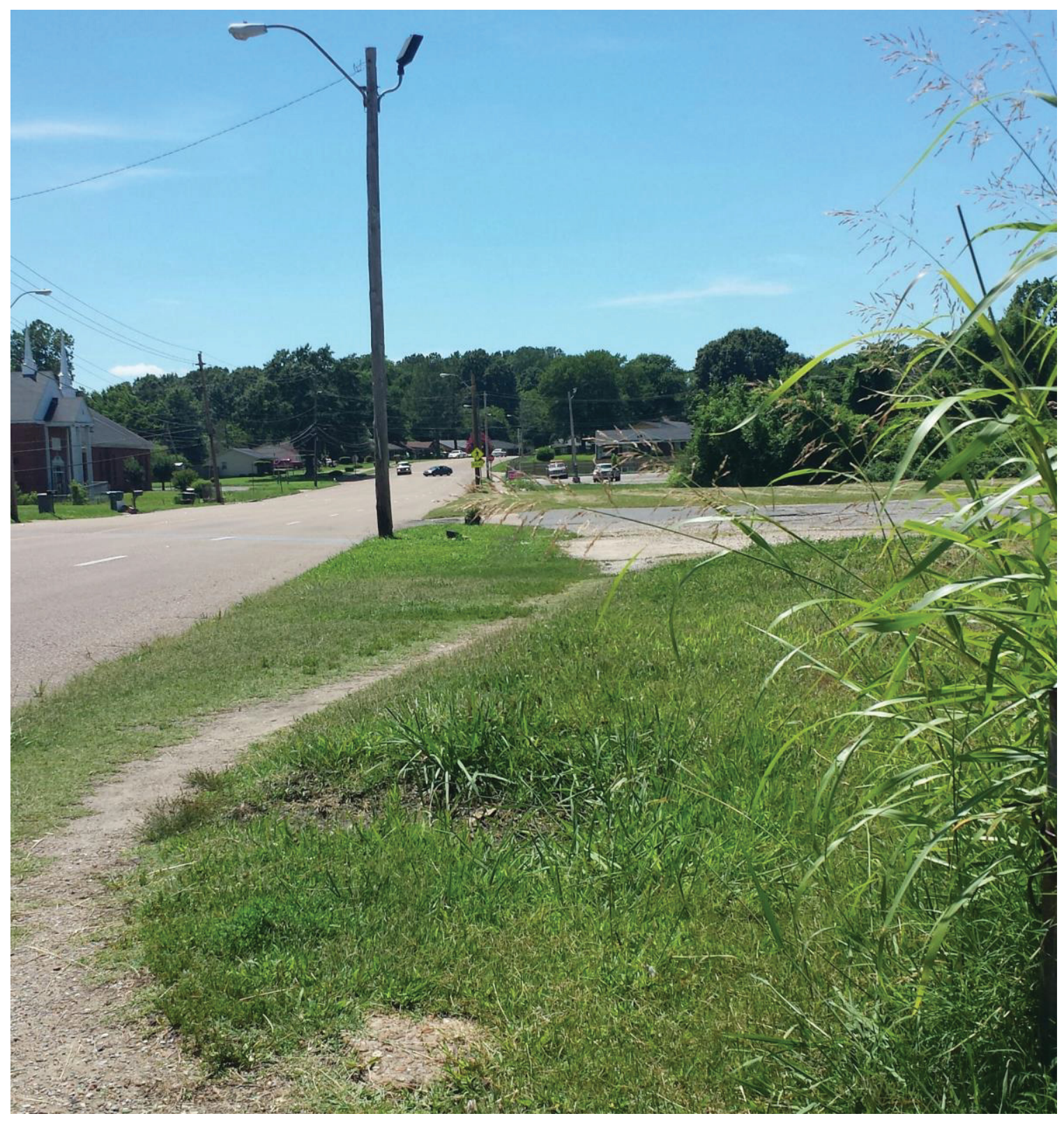




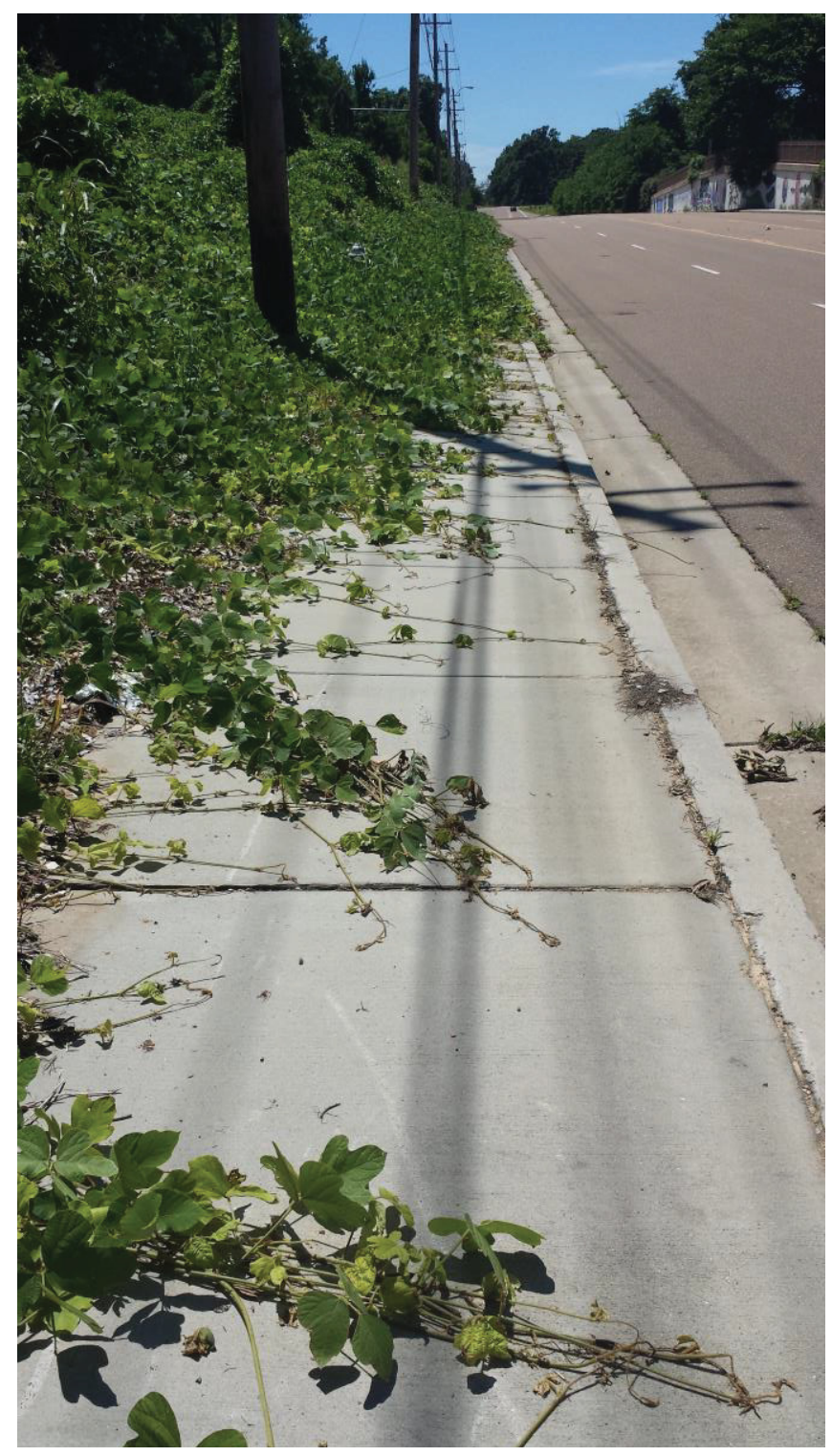




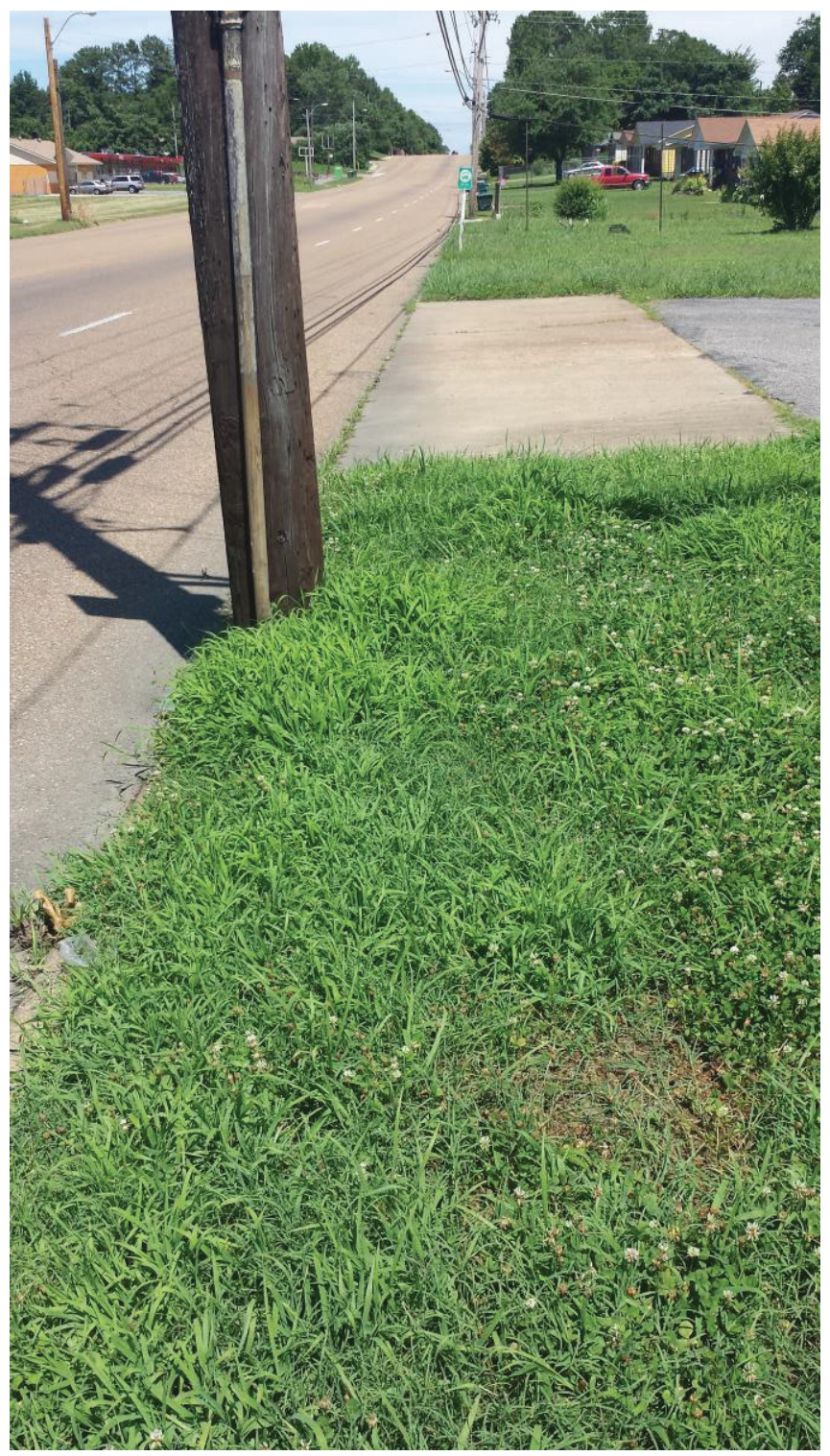




\section{APPENDIX B. RECRUITMENT LETTER}

\section{THEUNIVERSITYof \\ TENNESSEE \\ HEALTH SCIENCE CENTER}

October 22, 2013

$$
\begin{aligned}
& <<\text { Name of potential participant }>> \\
& <<\text { Address }>> \\
& <<\text { City, State, Zip }>>
\end{aligned}
$$

Re: Weight Loss Study among Women with Type 2 Diabetes, Conducted by Adole Muruako, Ph.D. Candidate

Dear $<<$ participant's name $>>$,

I am writing to let you know that a research study is being planned that may be of interest to you. It is possible that you may be eligible to participate in this study. Your eligibility can only be determined by the investigators of this study. The purpose of the study is to examine factors that aid in weight loss among type 2 diabetic women and to explore environment as a contributing factor. This study is being conducted by Adole Muruako, a Doctoral Candidate at the University of Tennessee Health Science Center.

The Endocrine Clinic is sending this letter on behalf of the University of Tennessee Health Science Center and has not shared any private information without the permission of the potential participant.

Please be aware that, even if you are eligible, your participation in this or any research study is completely voluntary. There will be no consequences to you whatever if you choose not to participate, and your regular medical care will not be affected by that choice. All information will be kept confidential. If you do choose to participate, the study will involve an eligibility questionnaire, which takes no more than 10 minutes, and an interview (if you are eligible) which is typically no longer than 30 minutes. All study activities are non-invasive. At the conclusion of all activities, you will receive a free $\mathbf{\$ 2 5}$ gift card.

In order to determine your eligibility and your interest in participating, you may call Adole Muruako, the Principal Investigator, at $(\mathbf{x x x}) \mathbf{x x x}-\mathbf{x x x x}$. You may also choose not to respond to this letter. If you do respond, any questions you have about the study will be answered.

Thank you for considering this research opportunity.

Sincerely,

The Endocrine Clinic

UTHSC

881 Madison Ave,

Suite 214 Memphis, TN 38103

iMedRIS Data Corporation

IRB NUMBER: 12-02151-XP

IRB APPROVAL DATE: 11/08/2013

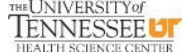




\section{APPENDIX C. RECRUITMENT FLYERS}

Have you been diagnosed with

TYPE 2 DIABETES

within the last 5 years?

- ARE YOU FEMALE

- BETWEEN THE $\boldsymbol{A} \boldsymbol{G E} \boldsymbol{O} \boldsymbol{O F}$ 35-49

- LIVE IN ONE OF THESE ZIP CODES: 38109, 38106, 38016, 38018, 38164

If so, you may be eligible to participate in a phone interview that seeks to understand the mechanisms of WEIGHT LOSS and environment.

At the completion of all activities, you will receive a FREE \$25 GIFT CARD.

Please contact Adole Muruako (xxx) xxxx-xxxx for more information and to determine eligibility. 


\section{Other important information}

- Either let the physician know about your interest in the study, or contact the study coordinator directly.

- The study coordinator will then contact you to determine the best times to conduct the interview.

- All information will remain confidential.

- The discussion will be audio recorded to capture important details.

- You are free to leave the study activities at any time.

- There are no physical risks associated with this study.

\section{About the study}

The purpose of this study is to examine factors that aid in weight loss among type 2 diabetic women and to explore

environment as a contributing factor.

This study is being conducted by Adole Muruako, a Doctoral Candidate at the University of Tennessee Health Science Center

\section{Contact Us}

Phone: [601-529-xxxx]

Email: [xxxx@uthsc.edu]

Web: http://www.uthsc.edu/COP

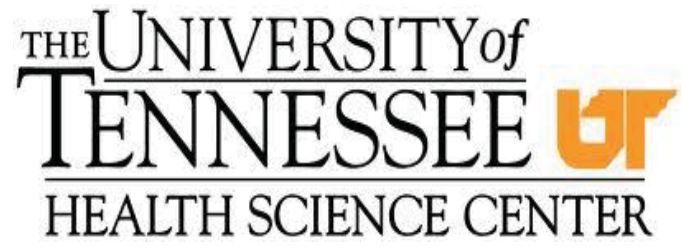

FREE \$25 GIFT CARD!!!!!!

UNIVERSITY OF TENNESSEE

\section{UNIVERSITY OF} TENNESSEE

881 Madison Ave, Suite 21448

Memphis, TN 38103
Conducted by Doctoral Candidate Adole Muruako, Graduate Student in the College of Pharmacy 


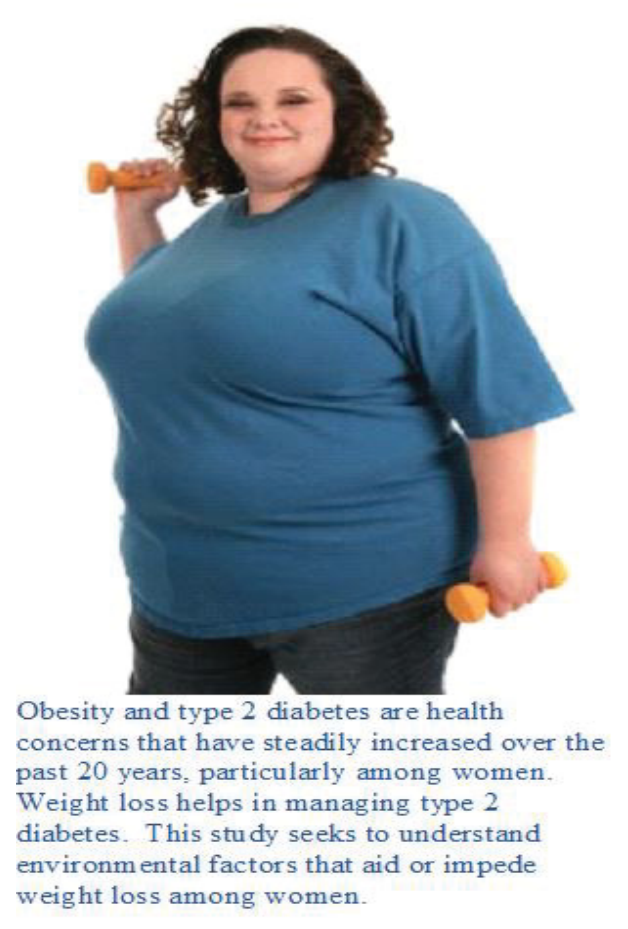

What will I be required to do to be part of this study?

To be part of this study, you must:

Be a woman, 35-49 years of age

Live in the following zip codes: 38109,38106 ,

$38016,38018,38164$

Be willing to participate in two interviews

(either by phone or in person), to determine

eligibility and answer study questions,

approxim ately 60 minutes of time.

Yes! I want to help!

All study-related activities are NON-

INVASIVE (no blood drawn).

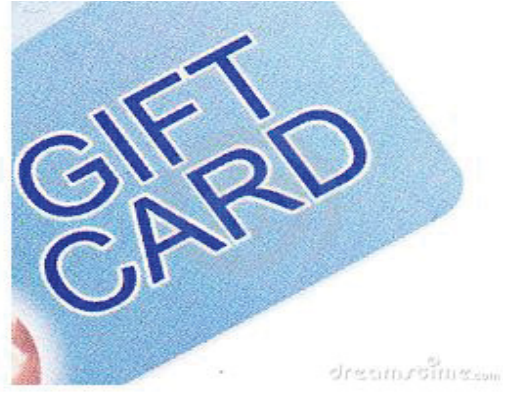

At the completion of all activities, you receive a FREE \$25 GIFT CARD to grocer 


\section{APPENDIX D. POTENTIAL PARTICIPANT SCREENING QUESTIONNAIRE}

Preparation date: 5/29/2012

Opening statement:

This interview is part of a research study. You are being given the opportunity to participate in this research study because you have been diagnosed with type 2 diabetes and may be interested in sharing your knowledge regarding weight loss. The purpose of this study is to describe things that may aid or may not aid women in successful weight loss while having type 2 diabetes. You will be asked to share your personal experiences. This study is being performed by Adole Muruako, a doctoral candidate at the University of Tennessee Health Science Center. This interview will last 60-90 minutes. You may be contacted to clarify statements you make afterward. The discussion will be audio recorded to capture important details. Your identity will be kept confidential. You may choose not to answer any questions at any time during this interview. You will receive a $\$ 25$ gift card once the interview is completed. You are free to leave at any time; however, gift certificates will be dispersed only after the entire interview session has been completed. There are no physical risks associated with this study. Participation is voluntary. You can choose to not participate; not participating will not affect your usual medical care. Risks associated with this study include potentially having uncomfortable feelings when completing the questionnaire or interview questions. There are also no benefits associated with participation in this study. Your experiences, however, will be used to determine the best and most influential possible ways to help women lose weight who have been diagnosed with type 2 diabetes.

Recruitment - Screening questions:

Question to identify type 2 diabetes patients:

Have you been diagnosed with type 2 diabetes? Yes No Year of diagnosis

Question to identify weight loss classification:

Have you purposely attempted to lose any weight since being diagnosed with diabetes? Yes No If so, when did you attempt to lose weight?

Have you lost any weight since being diagnosed with diabetes? Yes No If so, how much?

Have you gained any weight since being diagnosed with diabetes? Yes No If so, how much?

Weight at diagnosis:

Current weight:

Current height:

Question to address number in household:

How many persons reside in your household currently?

Question to identify additional comorbidities:

Have you been diagnosed with any other health condition? Yes No If yes, list condition

Question to identify whether or not they live in the areas of interest:

Do you live in either of the following zipcodes?

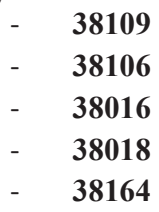

Question to identify income level (range):

What income level best describes you currently?

$\$ 0-15,000 \quad \$ 15,001-25,000 \quad \$ 25,001-50,000 \quad \$ 50,001-75,000 \quad \$ 75,000+$

One question to identify address

What is your current address?

One question to identify age What is your age? 


\section{APPENDIX E. SEMI-STRUCTURED INTERVIEW QUESTIONNAIRE}

\section{Semi-Structured Interview Guide}

1. Opening question: What has it been like having diabetes for you? Tell me your story.

2. Transition: How important is weight loss to you for your health?

3. Transition: What things do you do to help you lose weight and/or better manage your diabetes?

4. Key questions:

- Have you set weight loss or health maintenance goals?

What were they?

- Did you succeed?

- Has maintaining your health with diabetes been easy or difficult?

- What has been the financial impact of you trying to lose weight?

5. Transition question: How long have you lived in your neighborhood?

6. Key question: How do you feel about your neighborhood?

7. Key: How do you think your neighbors view exercise and taking care of their health?

8. Key: Do you exercise in your neighborhood to help you maintain your health?

a. Follow up if they do: How do you use your environment to better manage your diabetes? (examples: walking, running outside, walking around the neighborhood to get places, exercising with neighbors). Describe this situation.

b. Follow up if they don't: Is there any specific reason that you choose not to exercise in your neighborhood? (examples: blight, crime rates, lack of 
walkability, no knowledge of parks or trails, neighbors are not active, no recreational facilities, animals, lack of social ties)

7. Transition: (If they use the environment) What do you like about exercising in your neighborhood?

8. Key: What things in your neighborhood can be improved?

9. Conclusion: Is there anything else you'd like to add about exercising in your neighborhood that I didn't ask? 


\section{APPENDIX F. CONSTANT COMPARISON CHECKLIST}

- Are changes in the questioning route needed? If so, what changes are necessary?

- What personality characteristics were observed of the participant?

- What was observed of her home environment and the neighborhood?

- What did her body language indicate?

- Were there any inconsistencies noted in her reporting?

- What was the mood of the discussion?

- Were there any themes noted in responses to the key questions?

- Are there questions that need to be eliminated, revised, or added? 


\section{APPENDIX G. INSTITUTIONAL REVIEW BOARD FORMS}

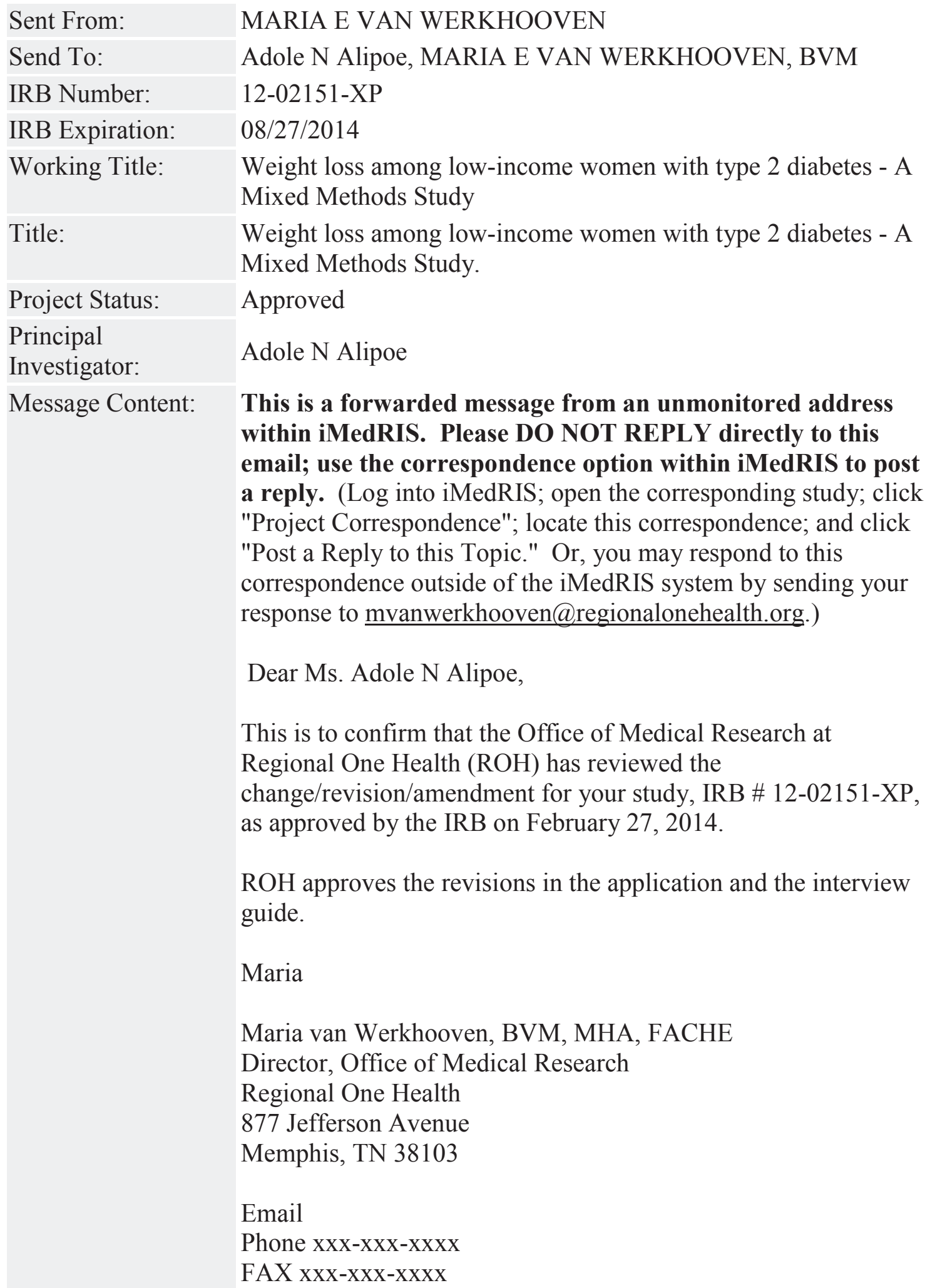




THE UNIVERSITY OF TENNESSEE
Health Science Center

Adole N Alipoe

UTHSC - COP - Pharmaceutical Sciences

214 Pharmacy Building

881 Madison Avenue

Memphis, TN 38163

Re: 12-02151-XP

Study Title: Weight loss among low-income women with Type 2 diabetes - A Mixed Methods Study.

\section{Dear Dr. Alipoe:}

The Administrative Section of the UTHSC Institutional Review Board (IRB) reviewed your application to continue your previously approved project, referenced above. It has determined that your application is eligible for expedited review under 45 CFR 46.110(b)(1), categories (5) and (7). The IRB reviewed your renewal application and determined that it does comply with proper consideration for the rights and welfare of human subjects and the regulatory requirements for the protection of human subjects. Therefore, this letter constitutes approval of your renewal application, including consent cover statement dated 08/19/2013 (stamped IRB approved 08/27/2013). In accord with 45 CFR 46.116(d), alteration of informed consent continues to be approved, with the cover statement used in lieu of an informed consent interview. The requirement to secure a signed consent form continues to be waived under 45 CFR 46.117(c)(2). Willingness of the subject to participate will constitute adequate documentation of consent. Approval of this study will be valid from 08/27/2013 to 08/27/2014.

This study may not be continued until you receive re-approval from the institution(s) where the research is being conducted.

In the event that subjects are to be recruited using solicitation materials, such as brochures, posters, web-based advertisements, etc., these materials must receive prior approval of the IRB. Any revisions in the approved application must also be submitted to and approved by the IRB prior to implementation. In addition, you are responsible for reporting any unanticipated serious adverse events or other problems involving risks to subject or others in the manner required by the local IRB policy.

Finally, re-approval of your project is required by the IRB in accord with the conditions specified above. You may not continue the research study beyond the time or other limits specified unless you obtain prior written approval of the IRB. 
THE UNIVERSITY OF TENNESSEE
Health Science Center

Adole N Alipoe

UTHSC - COP - Pharmaceutical Sciences

214 Pharmacy Building

Re: 12-02151-XP

Study Title: Weight loss among low-income women with Type 2 diabetes - A Mixed Methods Study.

Dear Ms. Alipoe:

The Administrative Section of the UTHSC Institutional Review Board (IRB) reviewed your application for revision of your previously approved project, referenced above.

The IRB determined that your application is eligible for expedited review under 45 CFR 46.110(b)(2). The attached revisions were approved as complying with proper consideration of the rights and welfare of human subjects and the regulatory requirements for the protection of human subjects. Approval does not alter the expiration date of this project, which is August 27, 2014.

The revisions to this study may not be instituted until you receive approval from the institution(s) where the research is being conducted.

In the event that subjects are to be recruited using solicitation materials, such as brochures, posters, webbased advertisements, etc., these materials must receive prior approval of the IRB. Any revisions in the approved application must also be submitted to and approved by the IRB prior to implementation. In addition, you are responsible for reporting any unanticipated serious adverse events or other problems involving risks to subjects or others in the manner required by the local IRB policy.

Finally, re-approval of your project is required by the IRB in accord with the conditions specified above. You may not continue the research study beyond the time or other limits specified unless you obtain prior written approval of the IRB.

Sincerely,

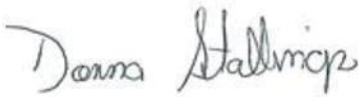

Signature applied by Donna L Stallings on 02/27/2014 08:53:30 AM CST

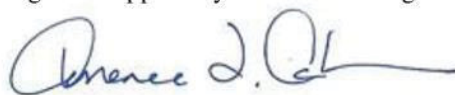

Signature applied by Terrence F Ackerman on 02/27/2014 08:54:12 AM CST

Donna Stallings, CIM

IRB Administrator

UTHSC IRB

Attachment: Revisions
Terrence F. Ackerman, Ph.D.

Chairman

UTHSC IRB 


\section{VITA}

Adole Natasha Muruako was born in Lubbock, Texas in 1984 to Dr. Dovi and Mrs. Theresa Alipoe. She moved to Mississippi with her family at 3 years of age. She is the first of three children: Michael and Eric. She attended Holy Family Elementary School and Cathedral Middle School in Natchez, MS. She moved to Vicksburg, MS in 1998, where she completed high school at Vicksburg High School in 2003. After graduation from high school, Adole was accepted into the summer bridge program at the University of Mississippi in Oxford, MS, where she also attended college to complete her undergraduate studies. During the summers of 2005 and 2006, she participated in the McNair Program at the University of Tennessee Health Science Center where she conducted research in the laboratory of Dr. Keith English and was introduced to the possibilities of pursuing a $\mathrm{PhD}$. She received a Bachelor of Science with a major in biology from the University of Mississippi in May 2007. In August 2008, she entered the Health Outcomes and Policy Research program at The University of Tennessee Health Science Center where she assisted with community health education and health screenings with the Consortium for Health Education, Economic Empowerment, and Research, as well as worked as an intern at Accredo Specialty Pharmacy. As a doctoral student, Adole conducted research on determinants of diabetes as a function of economics and environment. Adole, along with her husband Lawrence, established an entity entitled Operation Fit Nation, where they have hosted various events intended to interactively educate on matters of health and wellness. Adole is currently the Director of Wellness, Research, and Community Engagement at Tallahatchie General Hospital, where she coordinates efforts to expand and research community health, education, and healthcare resources in Charleston, MS and Tallahatchie County. Adole also works with The Works, Inc. in Memphis, TN, instructing healthy cooking classes for residents of the 38106 community with the Cooking Matters curriculum. She will receive her Doctor of Philosophy with a major in Health Outcomes and Policy Research in December 2014. 